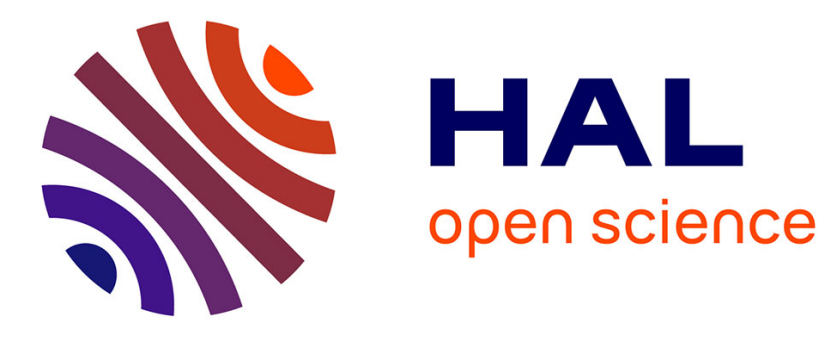

\title{
New Trends in Stochastic Geometry for Wireless Networks: A Tutorial and Survey
}

Yassine Hmamouche, Mustapha Benjillali, Samir Saoudi, Halim Yanikomeroglu, Marco Di Renzo

\section{- To cite this version:}

Yassine Hmamouche, Mustapha Benjillali, Samir Saoudi, Halim Yanikomeroglu, Marco Di Renzo. New Trends in Stochastic Geometry for Wireless Networks: A Tutorial and Survey. Proceedings of the IEEE, 2021, 10.1109/JPROC.2021.3061778 . hal-03149285

\section{HAL Id: hal-03149285 \\ https://hal.science/hal-03149285}

Submitted on 22 Feb 2021

HAL is a multi-disciplinary open access archive for the deposit and dissemination of scientific research documents, whether they are published or not. The documents may come from teaching and research institutions in France or abroad, or from public or private research centers.
L'archive ouverte pluridisciplinaire HAL, est destinée au dépôt et à la diffusion de documents scientifiques de niveau recherche, publiés ou non, émanant des établissements d'enseignement et de recherche français ou étrangers, des laboratoires publics ou privés. 


\title{
New Trends in Stochastic Geometry for Wireless Networks: A Tutorial and Survey
}

\author{
Yassine Hmamouche, Member, IEEE, Mustapha Benjillali, Senior Member, IEEE, \\ Samir Saoudi, Senior Member, IEEE, Halim Yanikomeroglu, Fellow, IEEE, and Marco Di Renzo, Fellow, IEEE
}

\begin{abstract}
Next generation wireless networks are expected to be highly heterogeneous, multi-layered, with embedded intelligence at both the core and edge of the network. In such a context, system-level performance evaluation will be very important to formulate relevant insights into tradeoffs that govern such a complex system. Over the past decade, stochastic geometry (SG) has emerged as a powerful analytical tool to evaluate system-level performance of wireless networks and capture their tendency towards heterogeneity. However, with the imminent onset of this crucial new decade, where global commercialization of fifthgeneration (5G) is expected to emerge and essential research questions related to beyond fifth-generation (B5G) are intended to be identified, we are wondering about the role that a powerful tool like SG should play. In this paper, we first aim to track and summarize the novel SG models and techniques developed during the last decade in the evaluation of wireless networks. Next, we will outline how SG has been used to capture the properties of emerging radio access networks (RANs) for 5G/B5G and quantify the benefits of key enabling technologies. Finally, we will discuss new horizons that will breathe new life into the use of SG in the foreseeable future. For instance, using SG to evaluate performance metrics in the visionary paradigm of molecular communications. Also, we will review how SG is envisioned to cooperate with machine learning seen as a crucial component in the race towards ubiquitous wireless intelligence. Another important insight is Grothendieck toposes considered as a powerful mathematical concept that can help to solve longstanding problems formulated in SG.
\end{abstract}

Index Terms-Fifth-generation (5G) and beyond fifthgeneration (B5G) networks, point process theory, signal-tointerference-and-noise-ratio, stochastic geometry.

\section{INTRODUCTION}

Stochastic geometry (SG) is a field of applied probability that aims to provide tractable mathematical models and appropriate statistical methods to study and analyze random phenomena on the plane $\mathbb{R}^{2}$ or in larger dimensions [1]. Its development was driven by applications in several scientific areas such as forestry, image analysis, geophysics, neurophysiology, cardiology, finance, and economics. In the context

Y. Hmamouche and S. Saoudi are with Mathematical and Electrical Engineering Department, IMT-Atlantique, Lab-STICC, UMR CNRS 6285, F-29238, France.

(e-mail: \{yassine.hmamouche, samir.saoudi\}@imt-atlantique.fr).

M. Benjillali is with the Department of Communication Systems, INPT, Rabat 10100, Morocco. (e-mail: benjillali@ieee.org).

H. Yanikomeroglu is with the Department of Systems and Computer Engineering, Carleton University, Ottawa, ON K1S 5B6, Canada. (e-mail: halim@sce.carleton.ca).

M. Di Renzo is with Université Paris-Saclay, CNRS, CentraleSupélec, Laboratoire des Signaux et Systèmes, 3 Rue Joliot-Curie, 91192 Gif-sur-Yvette, France. (e-mail: marco.di-renzo@universite-paris-saclay.fr).

This work was supported by Pôle de Recherche Avancée en Communications (PRACOM) and the Regional Council of Brittany, France.

\section{GLOSSARY}

$\mu$ Wave microwave

3GPP 3rd generation partnership project

AF Amplify-and-forward

AoI Age of information

ASE Area spectral efficiency

AtG Air-to-ground

AWGN Additive white Gaussian noise

B5G Beyond 5G

BBU Baseband unit

BPM Bounded path loss model

BPP Binomial point process

CAPEX Capital expenditure

CoMP Coordinated multipoint

C-plane Control plane

CR Cognitive radio

C-RAN Cloud radio access network

CRE Cell range expansion

D2D Device-to-device

DAS Distributed antenna system

dcx Directionally convex

DF Decode-and-forward

DPP Determinantal point process

DUDA Decoupled uplink-downlink access

EE Energy effeciency

EM Electromagnetic

eMBB enhanced mobile broadband

F-RAN Fog radio access network

FSO Free space optical

GPP Ginibre point process

GSPP Geyer saturation point process

HD Half duplex

HetNet Heterogeneous network

HPPP Homogeneous Poisson point process

IBFD In-band full-duplex

ICIC Intercell interference coordination

IDT Inhomogeneous double thinning

IoT Internet of things

IPPP Inhomogeneous Poisson point process

LED Light-emitting diode

LGCP Log-Gaussian Cox process

LOS Line-of-sight

MAC Medium access control

MC Molecular communication

MCMC Markov chain Monte Carlo

MCP Matérn cluster process

MGF Moment generating function

MHPP Matérn hard-core point process

MIMO Multiple-input and multiple-output

ML Machine learning

mmWave Millimeter wave

NLOS Non-line-of-sight

NOMA Non-orthogonal multiple access

OPEX Operational expenditure

PCP Poisson cluster process

PDF Probability density function

PGFL Probability generating functional 


\begin{tabular}{|c|c|}
\hline PHCP & Poisson hard-core process \\
\hline PHP & Poisson hole process \\
\hline PL & Perturbed lattice \\
\hline PLP & Poisson line process \\
\hline PMF & Probability mass function \\
\hline PP & Point process \\
\hline PPP & Poisson point process \\
\hline RAN & Radio access network \\
\hline RIS & Reconfigurable intelligent surface \\
\hline $\mathrm{RN}$ & Relay node \\
\hline ROI & Return on investment \\
\hline RRH & Remote radio head \\
\hline RWP & Random waypoint \\
\hline SDN & Software-defined networking \\
\hline $\mathrm{SG}$ & Stochastic geometry \\
\hline SI & Self interference \\
\hline $\mathrm{SIC}$ & Successive interference cancellation \\
\hline SINR & Signal-to-interference-plus-noise ratio \\
\hline SPP & Strauss point process \\
\hline SSI & Simple sequential inhibition \\
\hline SWIPT & Simultaneous wireless information and power transfer \\
\hline TCP & Thomas cluster process \\
\hline UAV & Unmanned aerial vehicles \\
\hline UDN & Ultra-dense network \\
\hline UE & User equipment \\
\hline U-plane & User plane \\
\hline UPM & Unbounded path loss model \\
\hline URLLC & Ultra-reliable and low latency communications \\
\hline VAD & Voronoi area distribution \\
\hline VANET & Vehicular ad hoc network \\
\hline VLC & Visible light communications \\
\hline
\end{tabular}

of communication networks, the location of user equipment (UE) and base stations (BSs) are scattered randomly over an enormous number of possibilities, where designing the system for every network realization would be time-consuming and resource-intensive [2], [3]. Instead, using tools from SG [1][8], the location of nodes (i.e., UEs and/or BSs) is assessed statistically in order to study the interaction between them, which inherently considers all possible network realizations and generally capture the main dependencies of the network performance connectivity (capacity/throughput and reliability). This is broadly understood if we see the concept of using a statistical distribution to abstract the variety of potential network topologies as actually similar to the approach of considering a statistical distribution to model the infinite possibilities of multipath fading and shadowing.

\section{A. A Brief History of Stochastic Geometry}

SG as a concept of geometric probability is a field that can be stretched back at least 300 years. Indeed, the bond between probability theory and geometry reverts back to the 18 th century when several challenging problems and imagined experiments raised by prominent mathematicians, pondering about the impact of varying randomly geometric forms on the probability of specific events. We quote particularly the Buffon's needle problem ${ }^{1}$ (1733), and afterwards questions related to Sylvester's four-point problem ${ }^{2}$ (1864) and Bertrand's

\footnotetext{
${ }^{1}$ Buffon's needle problem asks to find the probability that a needle of a given length will land on a line, given a window with equally spaced parallel lines far apart by a given distance. It provides a theoretical scheme to statistically determine the number $\pi$.

${ }^{2}$ Sylvester's four-point problem asks for the probability that four points scattered randomly in a given window region have a convex hull, i.e., it will be possible to connect any two points within the shape constructed by the four points with a straight line that does not leave the shape.
}

paradox $^{3}$ (1889). A short historical outline of these early days of geometric probability may be found in [9].

Since the 1950s, the framework of geometric probability broadened substantially and framed as an academic area. In particular, the focus mainly switched to models involving a typical number of randomly selected geometric objects. As a consequence, the four distinguishable mathematical strands of integral geometry theory [10], random set theory [11], random measures theory [12], and point process (PP) theory [4]-[8] started to play a prominent role in the geometric probability, which since then was called stochastic geometry. Integral geometry gives a unified approach for defining integrands over curves, surfaces, volumes, and higher-dimensional manifolds by using tools from probability theory, group theory, and projective geometry. Random sets generalizes the concept of random vectors, by addressing random entities whose number of components is unknown. Random measures theory is focused on studying the properties of measures established on random elements. In the special case where these measures are integer-valued, random measures reduce to PPs considered as an important subclass of random measures. Discussions on how early problems on geometrical probability have led to the construction of primary results on these pillar theories of SG, can be found in [13]. Moreover, for the sake of exploratory data analysis, parameter estimation, and model fitting, SG has been endowed with a statistical theory in similarity with the traditional probability theory. More statistical analysis and parameters estimation can be found in [14].

In the context of communication networks, the paper [15] is the first to consider tools from SG to evaluate connectivity in a network of stations represented by a Poisson point process (PPP). In particular, it was only by the late 1990s that important ideas from SG found their way to modeling and analysis of communication networks [2], [3], where tools based on Poisson Voronoi tessellations and Delaunay triangulation were proposed to derive geometric characteristics of hierarchical links between stations. To the best of the authors' knowledge, key results were reported a decade later, where the baseline mathematical framework was characterized in the case of a generative single-tier wireless network [16]-[18]. Since then, generalizations to more advanced SG models have been gradually adopted in subsequent works. For example, extensions to finite wireless networks are studied in [19], to multi-tier networks are reported in [20], [21], and to the uplink direction are analyzed in [22]. More discussions about such early extensions can be found in [23]-[26].

\section{B. The Importance of Stochastic Geometry in 5G/B5G Wire-} less Networks

With exponential digitalization of modern society, 5G/B5G networks are envisioned to play a major role in the process of achieving higher data rates, hyper-connectivity, and ultra-low latency [27], [28]. To achieve such requirements, future $5 \mathrm{G} / \mathrm{B} 5 \mathrm{G}$ wireless networks are expected to be more heterogeneous due to various targeted verticals with specific

\footnotetext{
${ }^{3}$ The Bertrand paradox asks for the probability that the chord of a circle will be longer than the side of an equilateral triangle inscribed in this circle.
} 
demand, in addition to the use of higher-frequency bands (e.g., mmWave [29], terahertz (THz) communications [30], and visible light communications (VLC) [31]) enabling to build high-speed short-range networks. Also, environmental objects will be coated with intelligent metasurfaces, commonly known as reconfigurable intelligent surfaces (RIS), which can reflect incident signals in a customized way to optimise/recycle signal propagation in future networks [32], [33]. The use of unmanned aerial vehicles (UAVs) will be a common and mature technology, where they can be used as flying-BSs to support terrestrial coverage in isolated regions, enhance capacity in traffic overloaded user hotspots, and even used as flying-UEs for delivery or supervision purposes [34]. Interestingly, end terminals will be gradually equipped with computing and/or storage capabilities, in a fog radio access architecture (F-RAN) fashion [35], enabling to emerge from the paradigm of ubiquitous connectivity to that of ubiquitous wireless intelligence.

Consequently, as the complexity and heterogeneity of modern wireless networks is continuously increasing, tools from artificial intelligence and machine learning (ML) will be crucial to learn static and dynamic components of the wireless environment and then help to make optimal control decisions for system-level performance. Also, SG adopted as a powerful model-driven tool for the evaluation of wireless networks during the last decade, is expected to remain an effervescent area of research in the foreseeable future, due typically to the following reasons: First, spatial arrangement of transmitters and receivers will continue to play a major role in the prediction of performance metrics in 5G/B5G wireless networks, e.g., performance scaling laws in ultradense networks (UDNs) [36], [37], impact of coupling UE and BS locations on system-level performance (see Table IV). Second, a cross-fertilization between SG and ML can be made to achieve better results in terms of accuracy and flexibility [38], [39]. For instance, SG can be integrated as a hypothesis class in the learning process of ML to evaluate the family of subset selection problems [40], [41]. Third, despite the ability to build a programmable and controlled wireless environment in 5G/B5G networks, thanks to F-RAN and massive adoption of metasurfaces, it is actually impossible to control all facets of the environment, e.g., building sway generated by winds and thermal expansion of materials [42] or beams misalignment in higher-frequency communications [43]. Hence, the need to model such uncontrolled network aspects with random processes, and then the ubiquitous need for SG.

\section{Relevant Surveys on Stochastic Geometry for Wireless Networks}

Given its mathematical flexibility and rich theoretical background, on the one hand, and massive proliferation of new communication concepts and technologies, on the other hand, several interesting survey and magazine papers have been developed on applications of SG in wireless networks [23][26], [44]-[49].

For instance, the work in [23] is the first paper to survey the main SG models and tools used in the evaluation of communication networks. A particular focus of the paper is given to earlier references up to 2008, where tools from SG have been particularly leveraged in: i) fixed line networks to derive the main statistical properties of cables connecting subscribers and concentration points. Such properties are next used to evaluate infrastructure costs as a function of nodes density, ii) cellular networks to evaluate the impact of network geometry on key performance metrics based on the signal-to-interference-plusnoise ratio (SINR) levels, e.g., service coverage, handover, and paging, and iii) ad hoc networks to study connectivity properties of the random graphs based on the SINR. The paper in [24] is a tutorial investigating how analytical tools from SG, percolation theory, and random geometric graphs can be applied to evaluate interference in large-scale ad hoc networks and hence derive the related performance metrics. The work in [25] is a comprehensive survey of the literature up to 2013 on modeling and analysis of cellular networks based on SG. The work focuses on multi-tier and cognitive cellular networks given their increased importance in future networks. However, since the publication of [25] quite a number of other radio access network (RAN) technologies have emerged, such as UAV-aided communication networks [34], cloud RAN (C-RAN) [50], [51], and F-RAN [35]. Also, other 5G/B5G technology enablers have flourished, such as non-orthogonal multiple access (NOMA) scheme [52], in-band full-duplex (IBFD) communications [53], and physical layer security [54].

The tutorial paper in [26] presented generative analytical techniques extensively used in the literature to derive the SINR distribution with an emphasis on Rayleigh fading and PPP distributed nodes. In [44], [45], the authors highlighted the rapid trend of modern wireless networks towards heterogeneity and complexity, where new modeling paradigm from SG will be crucial to capture the implications of such heterogeneity, e.g., cell association, uplink-downlink relationship, and nodes' mobility. Furthermore, since connectivity in wireless networks is tightly related to the distribution distance between the receiver and the serving node/s, together with the distribution of the interference power, the survey paper in [46] discussed key techniques used to derive the distance distribution when assuming two generative cases: i) Nodes scattered in the 2-dimensional plane $\mathbb{R}^{2}$ according to a PPP, and ii) nodes independently and uniformly distributed inside a bounded region of $\mathbb{R}^{2}$. Also, papers in [47], [48] reviewed the literature results on how $\mathrm{SG}$ models have been explored to capture the interference effect in ad hoc networks, while the work in [49] is a tutorial paper on how SG has been judiciously used to characterize interference in cellular networks.

\section{Our Contributions and Paper Organization}

This paper differs typically from prior papers in the following ways. First, we review the largely fragmented literature, up to $2020^{4}$, in wireless communication applications leveraging PP models, and provide for the first time a comprehensive taxonomy of them. While all the previous works focused on standard PP models, namely the PPP, the binomial point process

\footnotetext{
${ }^{4}$ All previous papers suggest over 200 citations, while this paper considers 424 representative references given the wide time frame and diversity of this paper scope.
} 
TABLE I

MAIN DIFFERENCES WITH PREVIOUS WORKS

\begin{tabular}{|c|c|c|c|c|c|c|c|c|}
\hline References & $\begin{array}{c}\text { Year of } \\
\text { publication }\end{array}$ & $\begin{array}{l}\text { No. of } \\
\text { examined PPs }\end{array}$ & $\begin{array}{l}\text { Statistical analysis } \\
\text { and PPs comparaison }\end{array}$ & $\begin{array}{l}\text { No. of surveyed } \\
\text { performance metrics }\end{array}$ & $\begin{array}{l}\text { No. of analytical } \\
\text { techniques }\end{array}$ & $\begin{array}{l}\text { No. of examined access } \\
\text { architectures }\end{array}$ & $\begin{array}{l}\text { No. of reviewed } 5 \mathrm{G} / \mathrm{B} 5 \mathrm{G} \\
\text { enabling technologies }\end{array}$ & $\begin{array}{l}\text { New avenues } \\
\text { to revitalize SG }\end{array}$ \\
\hline [23] & 2009 & (2) PPP and PLP & & $\begin{array}{l}\text { (3) Coverage probability, } \\
\text { paging, handover }\end{array}$ & 1 & $\begin{array}{l}\text { (3) Fix, ad hoc, and celluar } \\
\text { networks }\end{array}$ & & \\
\hline [25] & 2013 & $\begin{array}{l}\text { (3) PPP, BPP, } \\
\text { and PCP }\end{array}$ & & (1) Coverage probability & 5 & $\begin{array}{l}\text { (3) Ad hoc, single, and multi-tier } \\
\text { cellular networks }\end{array}$ & (1) CR networks & \\
\hline [26] & 2016 & (1) PPP & & (1) Coverage probability & 3 & $\begin{array}{l}\text { (2) Single and multi-tier cellular } \\
\text { networks }\end{array}$ & & \\
\hline This work & 2021 & (23) see Fig. 7 & (Yes) Section III $\sim$ IV & (13) see Fig. 10 & 11 & (6) Section VI & (10) Section VII & (4) Section VIII \\
\hline
\end{tabular}

TABLE II

A PROPOSED READING PATH TO GET STARTED WITH SG IN WIRELESS NETWORKS

\begin{tabular}{|c|c|c|c|c|c|}
\hline \multirow{2}{*}{ References } & \multirow{2}{*}{ Main topics } & \multicolumn{4}{|c|}{ Suitability to researchers } \\
\hline & & A concept primer & A technical primer & Intermediate reader & Advanced reader \\
\hline [44], [45] & $\begin{array}{l}\text { On the importance of using SG to grasp the implications of modern } \\
\text { networks tendency towards heterogeneity. }\end{array}$ & $\bullet$ & & & \\
\hline$[26]$ & $\begin{array}{l}\text { Generative analytical techniques to derive coverage probability under } \\
\text { the assumptions of Rayleigh fading and PPP distributed nodes. }\end{array}$ & & $\bullet$ & & \\
\hline [46] & $\begin{array}{l}\text { Generative techniques used to derive the transmit-receive distance } \\
\text { distribution. }\end{array}$ & & $\bullet$ & & \\
\hline [23] & SG models, up to 2008 , in the study of communication networks. & • & $\bullet$ & & \\
\hline$[24]$ & $\begin{array}{l}\text { Applications of SG, percolation theory, and random geometry in } \\
\text { interference characterization of ad hoc networks. }\end{array}$ & $\bullet$ & $\bullet$ & $\bullet$ & \\
\hline$[25]$ & $\begin{array}{l}\text { SG models, up to } 2013 \text {, in the study of heterogeneous and cognitive } \\
\text { networks. }\end{array}$ & $\bullet$ & & $\bullet$ & \\
\hline [47], [48] & Stochastic interference characterization in ad hoc networks. & & & $\bullet$ & \\
\hline [49] & Stochastic interference characterization in cellular networks. & & & $\bullet$ & \\
\hline$[1],[4]-[8],[10]-[12]$ & $\begin{array}{l}\text { SG theory in conjunction with related mathematical strands, e.g., } \\
\text { integral geometry theory, random set theory, and PP theory. }\end{array}$ & & & $\bullet$ & $\bullet$ \\
\hline This work & SG models, up to 2020 , in the study of wireless networks. & $\bullet$ & $\bullet$ & • & - \\
\hline
\end{tabular}

(BPP), and the Matérn hard-core point process (MHPP), this paper also addresses other powerful PPs given their inherent properties and system modeling implications (see Fig. 7). This paper additionally reviews the key statistical methods used to compare between PPs and fit some empirical data. Second, we examine the key choices taken in the work of SG system models. For instance, configurations used in the literature to capture coupling between nodes (see Table IV), various cell association strategies, and generative models to capture propagation effects. Also, novel performance metrics introduced after the publication of previous works, are discussed in this paper such as the meta-distribution and the update of coverage probability to account for signal strength/quality and handoff event (see Fig. 10). Next, we present in a refined tutorial fashion for non-specialists, the analytical techniques developed to date in the literature of SG-based modeling and analysis of wireless networks, where we delve into their key mathematical sequence steps. Third, we outline key modeling properties of new 5G/B5G technologies that have been extensively studied in the SG-based literature since the publication of the previous papers, including emerging RAN architectures such as multi-tier networks, infrastructure densification, UAVaided networks, C-RAN, virtualized RAN, and F-RAN, and other enabling technologies such as NOMA, higher-frequency bands, and IBFD. Finally, we usher in new avenues that will stimulate growth into the use of SG over this new decade.

Table I summarizes the key differences between our work and previous ones. To the best of the authors' knowledge, Table II suggests some key references to help a non-specialist reader familiarize with the field of using SG in wireless networks and keep the subsequently discussed concepts less arcane. Fig. 1 summarizes the content of this paper and for convenience, all abbreviations are listed in the Glossary.

Notation: $\mathbb{P}($.$) and \mathbb{E}($.$) stand for probability and expecta-$ tion measures. $\mathcal{L}_{X}(s)=\mathbb{E}\left(\mathrm{e}^{-s X}\right)$ is the Laplace transform of a random variable $X$ evaluated at $s$, sometimes referred to as the moment generating function (MGF). $\Phi_{X}(\omega)=\mathcal{L}_{X}(-j \omega)$ is the characteristic function (CF) of the random variable $\mathrm{X}$ such as $\sqrt{j}=-1$. We define for any reals $m, x \in \mathbb{R}$, $F_{m}(x)={ }_{2} F_{1}(1,-m ; 1-m ;-x)$ where ${ }_{2} F_{1}(., . ; . ; z)$ is the Gauss hypergeometric function for $z \in \mathbb{C}$. For $a, x \in \mathbb{R}$, $\Gamma(a, x)=\int_{x}^{\infty} t^{a-1} \mathrm{e}^{-t} \mathrm{~d} t$ is the upper incomplete Gamma function. We denote by $\psi^{-1}($.$) the inverse function of a$ function $\psi($.$) and \mathbb{1}($.$) is the indicator function.$

\section{STOCHASTIC GEOMETRY PRELIMINARIES}

In this section, we will discuss some core concepts of the PP theory that plays an important role in SG, since i) the building blocks of many important SG models are based on PPs as points are the most elementary types of geometrical objects; ii) it is common to parameterize geometric objects and map them with PPs in suitable state spaces, e.g., a line process in $\mathbb{R}^{2}$ can be seen as a PP on a cylinder [10]. Next, we will address the key properties of the PPP considered as the baseline and 


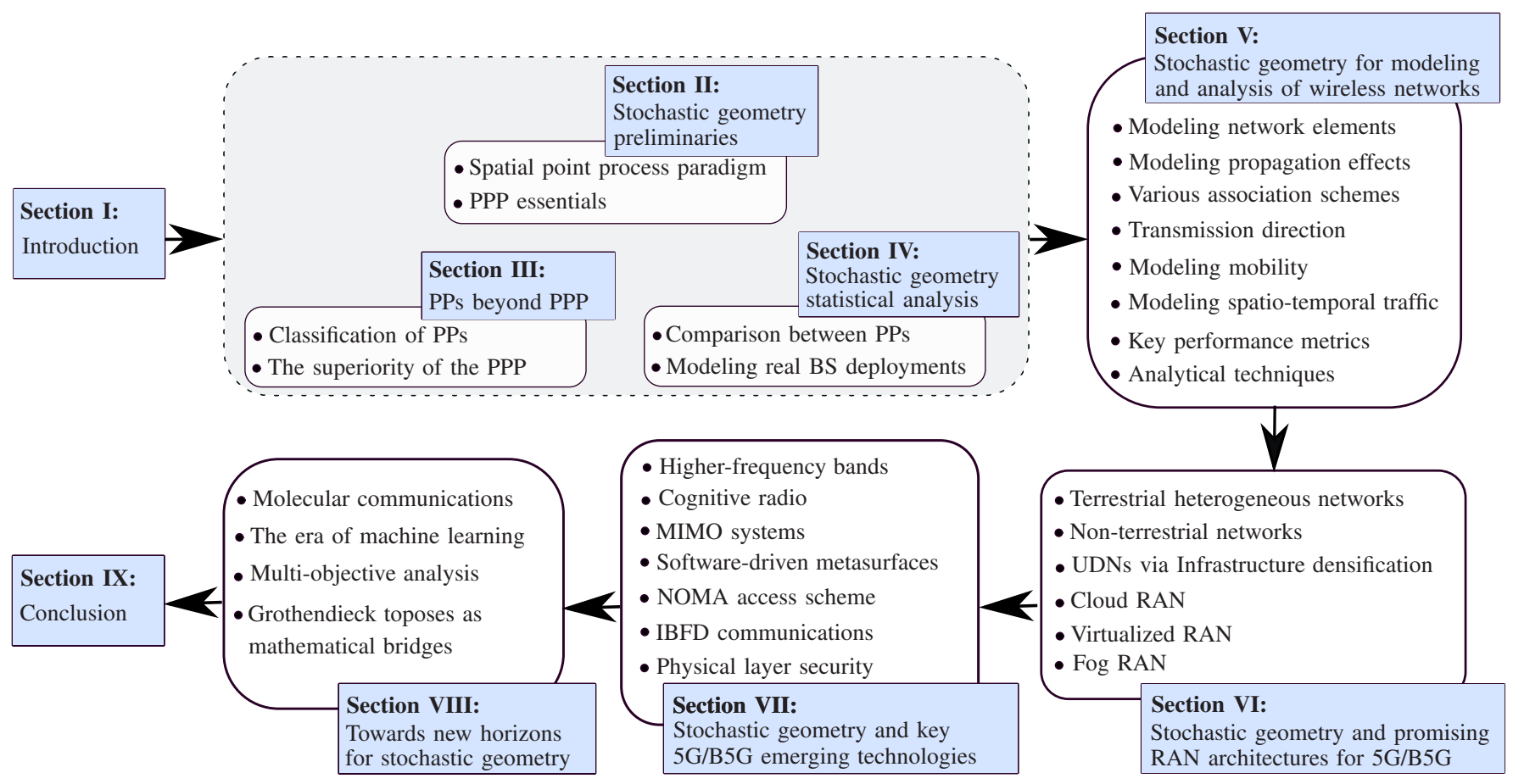

Fig. 1. Paper organization.

widely used PP, due to its practical mathematical attributes, where some key results can produce surprising consequences [2]-[4], [4]-[8].

\section{A. Spatial Point Process Paradigm}

In the context of communication networks, spatial PPs have become a burgeoning strand of SG models to evaluate the following aspects [4]-[8]

- The statistical properties of a given set of points.

- The possibility of having a point at a specific location.

- How to build a model of points with minimal error assumptions based on an empirical set of points.

- Describing more general random geometric objects made up of unit random elements.

1) The concept of point process: In contrast to earlier applications in queuing theory, where time has a natural order in one-dimensional PPs, the concept is quite different in higher dimensions $(d \geq 2)$, where there is no natural order of points. Instead, the most common way is to consider the cumulative counting process of a spatial PP $\Psi$, defined for each bounded set $B \subset \mathbb{R}^{d}$ as the number of points $x_{i}$ falling into $B$,

$$
\Psi(B)=\sum_{x_{i} \in \Psi(B)} \mathbb{1}_{B}\left(x_{i}\right)
$$

In a more general way, we consider the $n$th factorial moment measure given by

$$
\begin{aligned}
\mathbb{M}^{(n)}\left(A_{1}, \cdots, A_{n}\right)=\mathbb{E}\left(\Psi^{(n)}\left(A_{1} \times \cdots \times A_{n}\right)\right) \\
=\mathbb{E}\left(\sum_{x_{1}, \cdots, x_{n} \in \Psi}^{\neq} \mathbb{1}_{A_{1} \times \cdots \times A_{n}}\left(x_{1}, \cdots, x_{n}\right)\right) \\
=\int_{A_{1}} \cdots \int_{A_{n}} \varrho^{(n)}\left(x_{1}, \cdots, x_{n}\right) \mathrm{d} x_{1} \cdots \mathrm{d} x_{n},
\end{aligned}
$$

where $\neq$ indicates the sum over pairwise distinct $n$-tuples and $\varrho^{(n)}():. A_{1} \times \cdots \times A_{n} \rightarrow \mathbb{R}^{+}$is the product density function w.r.t. the Lebesgue measure. Without loss of generality and for notation simplicity, we consider that when $x_{i} \in \Psi, x_{i}$ will refer to a random variable that captures the potential location of the point $x_{i}$ in $\mathbb{R}^{d}$. However, when $x_{i}$ is used as a parameter of a PDF (or more generally of a product density function) inside a given integral for example, $x_{i}$ will refer to the integration variable over a bounded set covered by the PP $\Psi$.

2) The nearest neighbor distance and the contact distribution function: One important metric related to the cumulative counting process in (1), is the probability mass function (PMF) of $N(\cdot)$ defined as the probability that there will be exactly $n$ points inside $B$, i.e., $\mathbb{P}(N(B)=n)$. A particular type is the void probability defined as $\operatorname{void}(B)=$ $\mathbb{P}(N(B)=0)$. When $B=b(y, r)$ is the ball ${ }^{5}$ of radius $r$ and centered at the typical ${ }^{6}$ point $y, \operatorname{void}(b(y, r))$ can be interestingly interpreted as the probability that the distance

\footnotetext{
${ }^{5}$ A different shape may be taken instead of a ball depending on the dimension $d$ of the Euclidean plane and the isotropy of $\Psi$.

${ }^{6}$ In PP theory, the typical point of a PP $\Psi$ is often considered. In wireless networks analysis, it is termed as the typical UE for the downlink analysis and the typical BS for the uplink analysis. Formally, it is a point that has been chosen by a selection procedure in which each point in the process has the same chance of being selected [4], [6]
} 
between $y$ and the closest point of $\Psi$ is larger than $r$. In this way, when $y \in \Psi$, we talk about the nearest neighbor distance distribution $G_{y}($.$) defined as the distribution of the$ distance between $y$ and the nearest point of $\Psi \backslash\{y\}$. In simple probability terms,

$$
\begin{aligned}
G_{y}(r) & =\mathbb{P}(\mathrm{d}(y, \Psi \backslash\{y\}) \leq r \mid y \in \Psi) \\
& =\mathbb{P}(N(b(y, r) \backslash\{y\})>0 \mid y \in \Psi) \\
& =1-\mathbb{P}(N(b(y, r))=1 \mid y \in \Psi),
\end{aligned}
$$

where $\mathrm{d}(y, \Psi \backslash\{y\})$ is the distance between the fixed location $y$ and the nearest point of $\Psi$ except $y$.

When $y \notin \Psi$, we consider the contact distribution function $F_{y}($.$) that represents the smallest radius necessary for the ball$ centered at $y$ to contact a point in $\Psi$. Formally,

$$
F_{y}(r)=\mathbb{P}(\mathrm{d}(y, \Psi) \leq r)=1-\mathbb{P}(N(b(y, r))=0) .
$$

$G_{y}($.$) and F_{y}($.$) are important first order summary charac-$ teristics of a given PP [14] enabling to capture clustering or regularity in PPs. Typically, they are equal for the case of a totally random PP like the PPP, while $G>F$ for clustered PPs (Cox, Nymann-Scott, etc.), and $G<F$ for regular PPs (shifted regular lattices, hard-core, and soft-core repulsive PPs, etc.), as illustrated in Fig. 2. More discussions about PPs comparison and classification will be brought in the sequel.

3) The reduced Palm probability: We consider the typical point $y$ from a stationary PP $\Psi$ and we shift $\Psi$ such as $y$ lies at the typical fixed location $o$ (the origin). For a given set $B \subset \mathbb{R}^{d}, G_{y}($.$) can be seen as the ratio between the mean$ number of points except $y$ in the ball of radius $r$ and centered at $o$, and the mean number of points inside $B$. Formally, it is the ratio between the reduced Campbell measure expressed as $\mathbb{E}_{\mathrm{o}}^{!}(\Psi(B))=\mathbb{E}\left(\sum_{y \in \Psi \cap B} \mathbb{1}_{\mathcal{A}}\left(\Psi_{-y} \backslash\{y\}\right)\right)$, and the average number of points inside $B$ expressed as $\lambda \nu(B)$, where $\mathcal{A}$ is the event $N(b(y, r) \backslash\{y\})>0, \Psi_{-y}$ is the shifted PP $\Psi$ such as $y$ lies at $o$, and $\nu(B)$ is the Lebesgue measure or the $d$-dimensional volume of the subset $B$.

The previous interpretation of the nearest neighbor distance $G_{y}($.$) is called the reduced Palm probability measure denoted$ by $\mathbb{P}_{0}^{!}$as [1], [4], [6]-[8]

$$
\mathbb{P}_{\mathrm{o}}^{!}(\Psi \in \mathcal{A})=\frac{1}{\lambda \nu(B)} \mathbb{E}\left(\sum_{y \in \Psi \cap B} \mathbb{1}_{\mathcal{A}}\left(\Psi_{-y} \backslash\{y\}\right)\right),
$$

where the index $O$ is to mention the shifting of $\Psi$ towards $O$, the superscript ! is to refer that the typical point in the origin $o$ is not counted, i.e., $\mathbb{P}_{o}(\Psi \backslash\{y\} \in \mathcal{A})=\mathbb{P}_{o}^{!}(\Psi \in \mathcal{A})$, and $\Psi \in \mathcal{A}$ mentioning that $\Psi$ has the property $\mathcal{A}$.

4) The marked point process: A generalization of the PP $\Psi$ is the concept of marked PP where each point $x_{i} \in \Psi$ is assigned a further quantity $m_{x_{i}}$, called marks, that provides extra information on the object represented by $x_{i}$. For example, when considering a PP incorporating BSs, marks can be the coverage area of each BS $x_{i}$ [4], the fading gain between a BS $x_{i}$ and the typical UE [55], [56], or the BS tier in a multi-tier network [57].

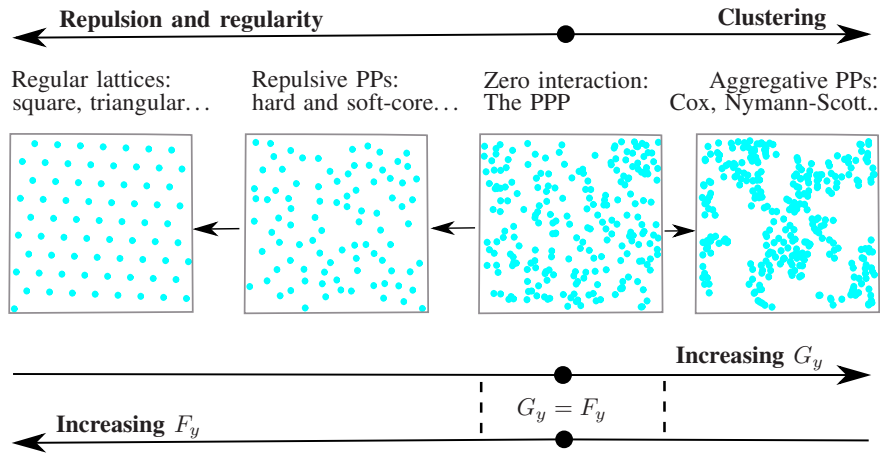

Fig. 2. The tendency towards regularity or clustering of PPs. Typically, increasing regularity reduces $G_{y}$ and increases $F_{y}$ simultaneously, while increasing clustering have a dual impact on $G_{y}$ and $F_{y}$.

\section{B. Poisson Point Process Essentials}

The PPP is considered as the most popular PP given its tractability and analytical flexibility [4]-[8]. In the following, we will discuss key properties underlying such tractability.

In general, a PPP $\Psi$ of density $\lambda($.$) and intensity measure$ $\Lambda($.$) such as for a given B \subset \mathbb{R}^{d}, \Lambda(B)=\int_{B} \lambda(x) \mathrm{d} x$, is characterized by a PMF as

$$
\mathbb{P}\{\Psi(B)=n\}=\frac{\Lambda(B)^{n}}{n !} \mathrm{e}^{-\Lambda(B)} .
$$

1) Slivnyak-Mecke theorem: For a homogeneous PPP (HPPP) $\Psi \subset \mathbb{R}^{2}$ with density $\lambda$, the number of points falling in disjoint Borel sets are independent. Hence, the points modeled by a HPPP are totally independent, that is why the HPPP is sometimes referred to as a zero-interaction PP [6]. In a more general way, the nearest neighbor distance distribution $G_{y}$ and the contact distance distribution $F_{y}$ are equivalent in the case of HPPP. Interestingly, this similarity can be seen as the equivalence between the reduced Palm probability of $\Psi$ in the typical point $y$ located at the origin $o$ and its original distribution counting $y$. In other words, the spatial averages observed at $o \notin \Psi$, are equivalent in distribution to those observed at $o$ of $\Psi \cup\{o\}$, which means that conditioning on the typical point does not affect the distribution of the PPP. This is the well-known Slivnyak-Mecke theorem [4]-[8], formally expressed as

$$
\mathbb{P}_{y}^{!}(\Psi \in .)=\mathbb{P}(\Psi \in .) .
$$

This theorem is extensively used in the literature. For instance, in a wireless network where the typical UE is located at the origin $o$, the Slivnyak-Mecke theorem can be used to derive the mean interference at $o$, conceiving that the serving BS $x_{0}$ belongs to the PP of interferers, but however, it does not contribute towards the interference [17], [18], [20], [21], [58]. Another valuable application is the transmit-receive distance distribution derived as in [4, Example 1.4.7] [16], [46], [59][61].

2) Finite Poisson point process: For a fixed number $n$ of nodes inside a given network area $W$, if $k \leq n$ nodes are located in a certain subset $B \subset W$, the remaining area $W \backslash B$ contains necessarily $n-k$ nodes, which introduces dependence between points of $W$, and hence the PPP is not so accurate to model such finite networks. Alternatively, the BPP 
is considered as the most relevant PP for such scenarios [19], [46], [62]-[64]. It is worth mentioning that according to (7), the probability that a point $x \in W$ belongs to $B$ is proportional to the number of points falling inside $B$. Equivalently,

$$
\mathbb{P}(x \in B)=\frac{\Lambda(B)}{\Lambda(W)} .
$$

In a more formal way, the conditional multivariate PDF $f\left(x_{1}, \ldots, x_{n} \mid \Psi(W)=n\right)$ defined w.r.t. the Lebesgue measure on $\left(\mathbb{R}^{d}\right)^{n}$ is expressed as

$$
f\left(x_{1}, \ldots, x_{n} \mid \Psi(W)=n\right)=\frac{\prod_{i=1}^{n} \lambda\left(x_{i}\right)}{\Lambda(W)^{k}} .
$$

Interestingly, the concept of (11) is explored to capture the structure of point patterns exhibiting inter-point interactions. That is, it is used in a more refined structure called the Papangelou conditional intensity to construct the family of Gibbs PPs [14], [65]-[67] and fitting statistical models to specific spatial point patterns via pseudo-likelihood maximization [68]. More generally, (11) is the building block in the definition of the reduced Palm distribution [8].

3) Simulation of Poisson point process: The equivalence property between a conditional PPP and a binomial distribution in a bounded window $W$, is typically used in simulation studies to generate a stationary PPP of density $\lambda$ [14], [65]. Practically, we first generate a Poisson variate $\mathcal{N}$ with parameter $\lambda \nu(W)$ and next we generate $\mathcal{N}$ independent and uniformly distributed (iud) points inside $W$. The resulted PP inside $W$ is equivalent to a PPP with density $\lambda$. Besides, (11) is considered as the key to generate an inhomogeneous PPP (IPPP). For example, we consider the realization of a 2dimensional IPPP with density $\lambda(x, y)=240\left(6 x^{5}+4 y^{3}\right)$ on the window $W=[0,1] \times[0,1]$. The PDF of a given point located in $(x, y)$ is $f(x, y)=\lambda(x, y) / 480$ bounded by 5 . Using the accept-reject method $\mathcal{N}$ times, where $\mathcal{N}$ is generated by a Poisson variate with parameter $\widetilde{\lambda}=480$, we draw uniformly $g$ on $[0,1]$ and accept $(x, y)$ such as $f(x, y) / 5 \leq g$. Fig. 3 describes the realization of the previous process in $W$. A valuable application of such technique in cellular networks modeling and analysis can be found in [69, Section VI].

4) Campbell and probability generating functional theorems: In the previous analysis, the PPP were constructed based on the PMF in (8). In the following, a PPP $\Psi$ can be constructed through probability densities on bounded subsets and generalizing the construction to the whole plane.

In fact, for any real positive function $f$ defined over $\mathbb{R}^{d}$, the probability generating functional (PGFL) of a PPP $\Psi$, named equivalently the Laplace functional, is expressed as [4]-[7]

$$
\begin{aligned}
\mathcal{L}_{\Psi}(f) & =\mathbb{E}\left(\exp \left(-\sum_{x_{i} \in \Psi} f\left(x_{i}\right)\right)\right) \\
& =\mathbb{E}\left(\exp \left(-\int_{\mathbb{R}^{d}} f(x) \Psi(\mathrm{d} x)\right)\right) \\
& \stackrel{(\mathrm{a})}{=} \exp \left(-\int_{\mathbb{R}^{d}}\left(1-\mathrm{e}^{-f(x)}\right) \Lambda(\mathrm{d} x)\right),
\end{aligned}
$$

where (a) follows by using the conditional PDF expression in (11) as in [4].

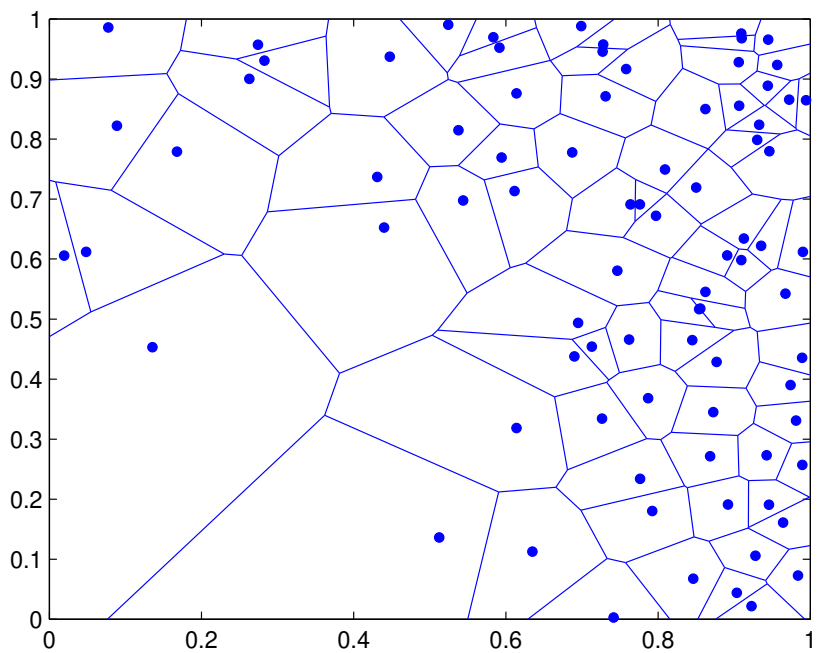

Fig. 3. Realization of a non homogeneous PPP on the window $W=[0,1] \times$ $[0,1]$ with density $\lambda(x, y)=240\left(6 x^{5}+4 y^{3}\right)$. Voronoi tessellation shows cells boundaries based on the spatially nearest points.

The expression (14) is considered to derive two fundamental results widely explored in SG-based modeling and analysis of wireless networks, namely the Campbell and PGFL theorems. In fact, by considering $t f(x) \rightarrow f(x)$ in (14) with $t \geq 0$ and differentiating w.r.t. $t$ at $t=0$, we obtain the Campbell theorem, as

$$
\mathbb{E}\left(\sum_{x_{i} \in \Psi} f\left(x_{i}\right)\right)=\int_{\mathbb{R}^{d}} f(x) \Lambda(\mathrm{d} x) .
$$

While by replacing $e^{-f(x)} \rightarrow f(x)$ in (14), we obtain the PGFL theorem for the PPP $\Psi$, as

$$
\mathbb{E}\left(\prod_{x_{i} \in \Psi} f\left(x_{i}\right)\right)=\exp \left(-\int_{\mathbb{R}^{d}}(1-f(x)) \Lambda(x)\right) .
$$

5) Preserving the Poisson law: Sometimes, it is necessary to consider some transformations on the PPP used to model node locations in order to obtain more insightful and tractable results. In the following, we consider popular operations preserving the Poisson law and extensively explored in the literature [4]-[8].

- Superposition: The union of independent PPPs $\left(\Psi_{k}\right)$ with intensities $\left(\Lambda_{k}\right)$ is a PPP $\Psi=\bigcup_{k} \Psi_{k}$ with intensity measure $\Lambda=\sum_{k} \Lambda_{k}$. As an illustration, the superposition of independent K-tier networks is investigated in [20], [21], [44], [58], [61], [70]. The superposition of two independent layers of line-of-sight (LOS) and non-lineof-sight (NLOS) BSs are considered in [71]-[73]. The superposition of independent PPPs to abstract the network of several competitive operators is considered in the context of infrastructure sharing [74], spectrum sharing [75]-[78], or both [79]-[81].

- Independent thinning: is a selection process $\Psi^{p}$ of specific points from the primary PPP $\Psi$ such that each point $x$ is randomly and independently selected with a probability $p(x)$. Accordingly, $\Psi^{p}$ yields a PPP of intensity measure equals to $\int_{\mathbb{R}^{d}} p(x) \Lambda(\mathrm{d} x)$ [4, Proposition 1.3.5.]. Typically, independent thinning is used to generate the family of Cox PP (e.g., Neymann-Scott, 
log-Gaussian) considered as a generalization of the PPP and used to capture clustered point patterns [1], [6], [82]. Also, the nodes of a given network can be thinned independently given their ability to be in LOS or NLOS transmissions with the typical UE [71]-[73], to operate in half-duplex (HD) or IBFD mode [83], or to use device-todevice (D2D) channels as in [84]. ALOHA, the popular algorithm used in the medium access control (MAC) layer to track simultaneous packet transmissions in the network, is considered in [4], [85] as an independent thinning of nodes willing to transmit data.

- Displacement: is a random transformation of points of $\Psi$ from $\mathbb{R}^{d}$ to some new location in $\Psi^{p}$ from $\mathbb{R}^{d^{\prime}}$ according to a probability $p$. The new PP inherits the Poisson law and its intensity measure is $\Lambda^{\prime}(A)=\int_{\mathbb{R}^{d}} p(x \in A) \Lambda(\mathrm{d} x)$, $A \subset \mathbb{R}^{d^{\prime}}$, as given by the displacement theorem [4, Theorem 1.3.9]. Valuable applications can be found in [4], [55], [86], [87]. In some settings, a given point $x \in \Psi$ may be moved deterministically with probability 1 into a function $f(x) \in \Psi^{f}[16]$, [61], and hence the new PP remains a PPP with intensity measure $\Lambda^{\prime}(A)=\Lambda\left(f^{-1}().\right)$. This property is also known as the mapping theorem [6]. A typical application is studied in [88], [89], where the authors considered an arbitrary path loss model and a generalized fading model, and next derived a sequence of equivalence relations between the so-called shotgun cellular system and a stochastically equivalent system, namely the canonical model.

\section{Point Processes Beyond the PPP}

Although the PPP model provides tractable results and many useful closed-form expressions, it cannot capture the geometry of real networks ${ }^{7}$ [67], [69], [90], [91], where nodes are negatively correlated, i.e., spatial inhibition and repulsion, or positively correlated, i.e., spatial aggregation and clustering. In fact, radio planning engineers are generally interested to deploy BSs on theoretical points where there will be a sufficient traffic demand and then an adequate return on investment (ROI). Hence, realistic deployments have commonly an increasing tendency towards clustering in user hotspots (e.g., events, urban area) and a tendency towards repulsion and regularity when users are equally likely scattered [92]-[94]. In this way, since the received SINR is sensitive to the interaction degree between nodes location, capturing the geometry of such nodes through an appropriate PP will directly impact the accuracy of network performance evaluation [45], [69], [92]-[96].

In the following, we will review the alternative PPs used in the literature to model the location of nodes exhibiting interaction and outline the key methods used to infer them. Also, we will discuss the relevant studies applying them in a variety of communication scenarios. Finally, we will develop a comprehensive classification of these PPs according to several

\footnotetext{
${ }^{7}$ By the end of this Section, we will discuss some recent research strands that endorse the ability of the PPP to capture the structure of real networks under some special setups (e.g., sufficiently strong log-normal shadowing, interference-limited regime, tendency of users towards clustering).
}

attributes such as the degree of interaction between points, the PP family, the ability to characterize interference at an arbitrary point when transmitters are scattered according to this PP, and the analytical tractability of such interference characterization.

\section{A. Classification of Point Processes}

A more universal way to classify PPs is by considering the interaction degree between points. In fact, point locations can interact negatively with each other to build a wellcrafted and regular structure or even an intermediate repulsive structure that can be either hard-core or soft-core. Conversely, a decrease in repulsion may be equivalent to an increase in randomness and then a tendency towards the paradigm of zero interaction PP, i.e., the PPP. Afterwards, a positive interaction between points will induce clustered points (see Fig. 2).

1) Stationary deterministic lattices: Traditionally, deterministic lattices, e.g., regular hexagonal lattice, or perfect square lattice, are often considered as a ubiquitous assumption in academia and research to model the location of nodes in a wireless network [47], [91]. Formally, a 2-dimensional stationary regular lattice can be expressed under the form

$$
\Lambda_{\text {grid }}=\left\{c G+U: c \in \mathbb{Z}^{2}\right\},
$$

where $\mathrm{G}$ is the generator matrix of the grid and $U$ is a uniformly distributed random vector over the Voronoi cell of the origin to ensure the stationarity of lattices.

However, despite the main advantage of regular lattices, where it is generally more efficient to design good channel access schemes as compared to networks where node locations are perceived as random or in motion, tractable network performance evaluation is only possible for specific user locations in the cell (cell edges, etc.), and a generalization over the entire cell requires complex and time-consuming Monte Carlo simulations [17], [18]. Also, with the proliferation of heterogeneous networks $\left(\mathrm{HetNets}^{8}\right.$ ) where cells radii vary considerably with differences in transmission power, grid models are seen as very idealized, yielding very optimistic results of performance evaluation [17], [18]. Typically, when comparing the results obtained from the PPP and lattice models with real deployments, we observe that the PPP model provides a lower bound of reality, while perfect lattices give an upper bound. An accurate PP lies then somewhere between the two extremes. It is neither perfectly periodic, nor completely independent.

2) Hard-core point processes: In such a family of PPs, there are no points at a distance smaller than a specific minimum threshold $\delta$, also known as the hard-core distance. In the following, we will discuss the key variants of hard-core PPs.

- Matérn hard-core point process (MHPP): There are generally two popular variants of MHPP used in the literature of wireless networks modeling and analysis, namely MHPP type I and MHPP type II [97]. MHPP I deletes all pairs of points with pairwise distance less than $\delta$ such that the density of the resulting PP is $\lambda=\lambda_{\mathrm{p}} \exp \left(-\pi \lambda_{\mathrm{p}} \delta^{2}\right)$,

\footnotetext{
${ }^{8}$ There is a slight abuse of meaning with the term of non homogeneous networks modeled by a PP with location-dependent density.
} 
where $\lambda_{\mathrm{p}}$ is the density of the parent PPP. In MHPP II, the process MHPP I is changed into a dynamic scheme by considering the parent PPP as marked by the uniform speed arrival times $t \in[0,1]$, which results on a density $\lambda=\left[1-\exp \left(-\lambda_{\mathrm{p}} \pi \delta^{2}\right)\right] / \pi \delta^{2}$. For $\lambda_{\mathrm{p}} \rightarrow \infty$, we note that MHPP I suggests that no point could survive after the dependent thinning process, while MHPP II predicts that the remaining points are correlated with $1 / \pi \delta^{2}$. Performance analytical evaluation of networks modeled by the MHPP is generally challenging given the reduced tractability of the contact and nearest neighbor functions, which enables to only derive approximations for the mean and the MGF of the interference. For instance, tight bounds of the mean interference in MHPP I and MHPP II wireless networks are investigated in [98], [99] and the contact distribution is evaluated in [100]. Interestingly, MHPP II is exploited in the literature to capture the minimum safe distance between vehicles in vehicular ad hoc networks (VANETs) [101]. More theoretical analysis of the MHPP can be found in [1], [97].

- Simple sequential inhibition (SSI): Another fashion to capture point patterns that exhibit inhibition, is by exploring sequential PPs, in which points are added one by one based on a given sequence. The most popular sequential process is the simple sequential inhibition (SSI) process, where each point is generated uniformly in a given window and independently from the previous points. The added point is rejected if it lies closer than the hard-core distance from the previous accepted points, and retained otherwise. Next, another point is generated and the process is ended if and only if we achieve the desired number of points inside the window or no other point can be added. A representative example is the work in [102], where the amount of regularity in MHPP I, MHPP II, and SSI is evaluated through some regularity metrics.

- The family of Gibbs point process-Poisson hard-core process (PHCP): An alternative way to capture point patterns that exhibit repulsion, is to proceed through the multivariate PDF in (2), which renders the construction and interpretation of the PP simpler, in addition to flexible simulations. Typically, the simulation of the multivariate PDF can be approximated for example by considering the equilibrium distribution of a Markov chain, also known as Markov Chain Monte Carlo (MCMC) algorithms, e.g., Métropolis-Hastings algorithms [14, Page 149]. A representative family of PPs based on this approach is the class of Gibbs PPs, also known as Markov PPs [103]. Formally, for a given finite spatial point pattern $u=$ $\left\{x_{1}, \ldots, x_{n(u)}\right\}$, the multivariate PDF of a finite Gibbs PP $\Psi$ is expressed as

$\varrho^{(n)}(u)=\exp \left(V_{0}+\sum_{i=1}^{n(u)} V_{1}\left(x_{i}\right)+\sum_{i<j \leq n(u)} V_{2}\left(x_{i}, x_{j}\right)+\ldots\right)$,

where $\exp \left(V_{0}\right)$ is a normalizing factor ensuring that $\varrho^{(n)}($.$) is a PDF, and for k \geq 1, V_{k}$ is a function reflecting the interaction order between points.
It is worth mentioning that the exponential form in (18) is not arbitrary but driven by the formulation of a maximization problem of the entropy in physics, expressed generally on the basis of logarithmic functions. Typically, statistical analysis has shown that the pairwise interaction is generally sufficient to model inter-points interaction [14]. In this way, the Gibbs PP is commonly known as pairwise interaction PP. The multivariate PDF of a stationary Gibbs PP $\Psi$, i.e., $V_{1}(x)=\log (\beta), \forall x \in u$, is simplified as

$$
\varrho^{(n)}(u)=\kappa \beta^{n(u)} \prod_{i<j \leq n(u)} h\left(\left\|x_{i}-x_{j}\right\|\right),
$$

where $\left\|x_{i}-x_{j}\right\|=\mathrm{d}\left(x_{i}, x_{j}\right), \kappa=\exp \left(V_{0}\right)$, and $h($. is a function dependent on the mutual distance between points.

The Poisson hard-core process (PHCP) is established as a special case of the Gibbs PP, such that $\forall x_{i}, x_{j} \in u$,

$$
h\left(\left\|x_{i}, x_{j}\right\|\right)= \begin{cases}1 & \text { if }\left\|x_{i}-x_{j}\right\|>\delta \\ 0 & \text { if }\left\|x_{i}-x_{j}\right\| \leq \delta .\end{cases}
$$

In wireless networks modeling and analysis, the PHCP was initially investigated via simulations in pattern recognition of deployed nodes that exhibit repulsion [67]. Some analytical investigations of the PHCP are next considered, to approximate for example performance metrics of a two-tier HetNet as in [104].

- Poisson hole process (PHP): Another way to conceptualize hard-core repulsion between points is to consider independent realizations of two HPPPs $\Psi_{1}$ and $\Psi_{2}$, with respective densities $\lambda_{1}$ and $\lambda_{2}$. Next, a PHP $\Psi$ is conceived by considering $\Psi_{2}$ as a parent PP depriving it of points located in holes (exclusion regions) of radius $\delta$ around the points of $\Psi_{1}$. The density of $\Psi$ is then expressed as [6], $\lambda=\lambda_{2} \exp \left(-\pi \lambda_{1} \delta\right)$. PHP belongs to the family of Cox PPs, i.e., doubly stochastic PPPs, where it is roughly considered as capturing clustering rather than inhibition, this is well understood since creating holes in one region forces nodes to cluster in other regions.

Several valuable applications of the PHP are reported in the literature. For instance, in cognitive networks [105], the holes are interpreted as the guard regions around primary users, where the PHP models secondary users allowed to transmit as long as they are located outside the holes, which reduces the detrimental effect of interference. In HetNets [104], [106], the PHP is explored to capture dependence between tiers, where small cells are not allowed to be deployed very close to macro cells. In [107], the authors proposed the use of the PHP to model a multi-cell D2D underlaid cellular network. Generally, despite the flexible construction of the PHP as compared to previous hard-core PPs, a complete characterization of interference and then SINR distribution is unfeasible. To overcome such limitation, two approaches are considered in the literature: i) Derive relatively tight bounds and approximations of the MGF of the interference [105], [107], [108], or ii) approximate the PHP realization with either a PPP or a tractable clustering PP [6], [106]. 
3) Soft-core repulsive point processes: A smooth way to generate inhibition between points is to increase the tendency towards repulsion and regularity without setting a deterministic restriction via hard-core distances. In the following, we will outline the key soft-core PPs used in the literature of wireless networks modeling and analysis.

- The family of Gibbs point process-Strauss point process $(S P P)$ : It is a special case of Gibbs PPs by defining for a constant $0<\gamma<1$, the function $h$ in (19), as

$$
h\left(\left\|x_{i}, x_{j}\right\|\right)= \begin{cases}1 & \text { if }\left\|x_{i}-x_{j}\right\|>\delta \\ \gamma & \text { if }\left\|x_{i}-x_{j}\right\| \leq \delta .\end{cases}
$$

The multivariate PDF in (19) is then simplified as

$$
\varrho^{(n)}(u)=\kappa \beta^{n(u)} \gamma^{s_{\delta}(u)},
$$

where $s_{\delta}(u)$ counts the number of unordered pairs of distinct points in $u$ spaced apart by less than $\delta$.

When $s(u)$ increases, the PDF in (22) is integrable and goes towards 0 , which decreases the tendency towards clustering. $\gamma$ helps then to softly adjust the repulsiveness intensity, where the SPP is typically reduced to a PPP when $\gamma=1$ and to a PHCP when $\gamma=0$. Practically, it is revealed in [66], [67] that the SPP is an optimal candidate for point patterns that exhibit a tendency towards repulsion. However, despite its easy interpretation and construction, the finite SPP is geared towards timeconsuming simulations and does not have closed-form expressions for the moments, while generalizations to infinite Gibbs PP renders the analysis even more complicated.

- Determinantal point processes (DPPs): To overcome the previous limitations of SPP, a DPP $\Psi$ acting over a given Borel $B \subseteq \mathbb{C}^{d}$ is constructed in such a way that it preserves the smart structure of Gibbs PPs in (19), but with a closed-form multivariate PDF w.r.t. the Lebesgue measure on $(\mathbb{C}, \mathcal{B}(\mathbb{C}))$ [109]-[115]. In this way, for two functions $h: B^{n} \rightarrow \mathbb{R}^{+}$and $C: B^{2} \rightarrow \mathbb{C}$, we have

$$
\begin{aligned}
& \mathbb{E}\left(\sum_{x_{1}, \ldots, x_{n} \in \Psi}^{\neq} h\left(x_{1}, \ldots, x_{n}\right)\right)= \\
& \int_{B} \ldots \int_{B} \varrho^{(n)}\left(x_{1}, \ldots, x_{n}\right) h\left(x_{1}, \ldots, x_{n}\right) \mathrm{d} x_{1} \cdots \mathrm{d} x_{n}, \\
& \text { and } \varrho^{(n)}\left(x_{1}, \ldots, x_{n}\right)=\operatorname{det}\left(C\left(x_{i}, x_{j}\right)\right)_{1 \leq i, j \leq n}, \quad
\end{aligned}
$$

where $\neq$ denotes that the finite points are pair-wise distinct, $\operatorname{det}($.$) denotes the determinant function, and the$ matrix $C$ is called the kernel of the DPP.

The repulsiveness of the DPP $\Psi$ stems from the observation that the determinant of a complex covariance matrix cannot be greater than the product of its eigenvalues [115], and then $\varrho^{(n)}\left(x_{1}, \cdots, x_{n}\right) \leq \prod_{i=1}^{n} \varrho^{(1)}\left(x_{i}\right)$, where equality holds in a PPP. Furthermore, motioninvariance of $\Psi$ implies that the kernel $C_{0}$ is real depending only on the distance between pairs of points.
$R=0.02$

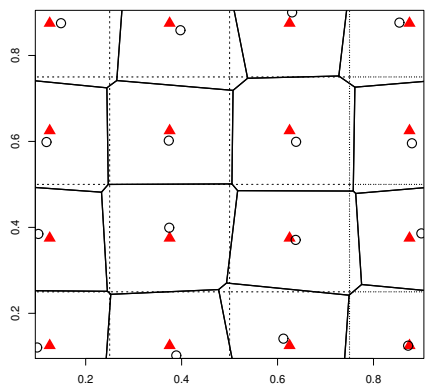

$R=0.08$

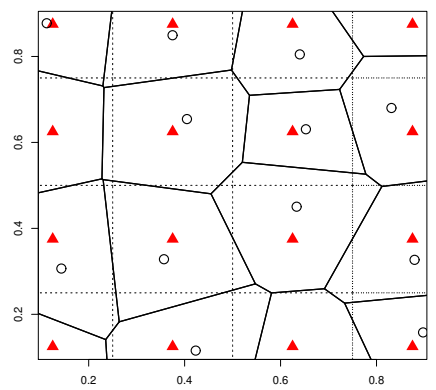

Fig. 4. Two realizations of the square lattice with uniform perturbation on the disk $\mathrm{b}(0, R)$. The dotted lines reflect borders of the Voronoi tessellation of the square lattice without perturbation (red triangles) and the solid lines that of the Voronoi tessellation of the perturbed lattice (circles).

That is, its Fourier transform, i.e., spectral density, exists and is defined as

$$
\varphi(x)=\mathcal{F}\left(C_{0}\right)(x)=\int_{B} C_{0}(y) e^{-2 \pi j x y} d y,
$$

where the existence of the associated DPP $\Psi$ to $C_{0}$ is constrained by checking $|\varphi| \leq 1$ [113, Proposition 5.1]. Depending then on the formulation of the covariance function $C_{0}$ or the spectral density $\varphi$, several versions of motion-invariant DPPs are constructed with different levels of repulsiveness and tractability [109], [110], [115]. For instance, $\Psi$ is a Gauss DPP if for every $u \in \mathbb{R}^{2}, C_{0}(u)=\lambda \exp \left(-\|u / \gamma\|^{2}\right)$, where $\lambda$ is the spatial intensity of the Gauss DPP and $\gamma$ is a parameter to adjust the repulsiveness of the DPP, such as $\pi \lambda \gamma^{2} \leq 1$ for the existence condition. The Cauchy DPP is obtained when $C_{0}(u)=\lambda /\left(1+\|u / \gamma\|^{2}\right)^{m+1}$ and an existence condition such that $\pi \lambda \gamma^{2} \leq m$, where $\lambda$ is the intensity of the process and $\alpha$ and $m$, are shape parameters to tune repulsiveness. The generalized Gamma DPP is defined with a spectral density $\varphi(u)=\lambda\left(m \gamma^{2} /(2 \pi \Gamma(2 / m))\right) \exp \left(-\|u \gamma\|^{m}\right)$, where the existence condition is $\lambda m \gamma^{2} \leq 2 \pi \Gamma(2 / m)$.

For more tractability and mathematical convenience, another form of motion-invariant DPPs is introduced, namely the scaled Ginibre PP ( $\beta-$ GPP), by considering a kernel as $C(x, y)=c \pi^{-1} \mathrm{e}^{-\frac{c}{2 \beta}\left(|x|^{2}+|y|^{2}\right)} \mathrm{e}^{\frac{c}{\beta} x \bar{y}}$, where the resulting density $\lambda$ is scaling with $c$ as $\lambda=c / \pi$ [111]-[114] and $\beta$ to seamlessly adjust the repulsion intensity. It is worth mentioning that in addition to the availability of closed-form moments of DPPs, a scaled $\beta-\operatorname{GPP} \Psi=\left(X_{i}\right)_{i \in \mathbb{N}}$ enhances mathematical tractability due to the fundamental property in which $\left(X_{i}^{2}\right)_{i \in \mathbb{N}}$ are mutually independent and follow a $\Gamma(i, \beta / c), \forall i \in \mathbb{N}$ [113, Proposition 1].

Relevant applications of the DPP have been reported in the literature. For instance, the authors of [109] investigated the goodness-of-fit of a real deployment scenario of nodes with three motion-invariant DPPs: the Gauss model, the Cauchy model, and the Generalized Gamma model, where it is revealed that the latter provides the best fit accuracy at the expense of reduced tractability due to the spectral density based construction of the model. Analytical investigation of the scaled $\beta-$ GPP is explored 
in [111]-[114], where tractable expressions of the contact distribution function and the Ripley's $K$-function (see next paragraphs) are derived; however, the distribution of SINR is yet of intractable formulation.

- Perturbed lattice (PL): At this stage, the above-discussed repulsive PPs differ in terms of their construction approaches, tractability, and capability to fit real deployment scenarios. However, one common shortcoming is their inability to softly capture point patterns that exhibit perfect regularity. Accordingly, the perturbed lattice (PL) is adopted in the literature of wireless networks modeling and analysis, such that the degree of perturbation allows to tune softly the process from a deterministic lattice (no perturbation) to highly random deployments (i.e., PPP) [55], [66], [67], [116]-[120]. Formally, the construction of a perturbed lattice $\Lambda_{\text {pert }}$ is based on (17), as

$$
\Lambda_{\text {pert }}=\Lambda_{\text {grid }}+X_{c}=\left\{c \mathrm{G}+U+X_{c}: c \in \mathbb{Z}^{2}\right\},
$$

where $X_{c}, c \in \mathbb{Z}^{2}$, is a family of i.i.d. random variables, uniformly distributed on a disk of radius $R$. In other words, $R$ is a control knob to tune the degree of perturbation (see Fig. 4). $R$ needs generally to be upper-bounded to avoid collision between nodes after perturbation, e.g., in the case of triangular lattice, $R$ needs to verify $0 \leq R<r_{s} \sqrt{3} / 2$ where $r_{s}$ is the radius of the circumscribed circle of the perfect lattice.

Given its ability to capture wide range of point patterns between the PPP and deterministic lattices, the PL is extensively investigated in the literature of wireless networks modeling and analysis. For instance, analytical bounds of the average interference and signal-tointerference ratio (SIR) distribution are studied in [116], [117]. In [66], [67], [117], [118], the PL is used to model realistic node deployments that exhibit repulsion. Interestingly and given the observation that the best SINR distribution is achievable under perfect lattices [18], the authors of [119], [120], proposed to proceed on the basis of a novel algorithm, namely the triangular lattice fit, to deactivate some empty BSs, i.e., BSs serving no UE, in such a way to render the structure of activated BSs as regular as possible, which will enable to maximize the overall performance.

Evaluating the amount of regularity in the location of transmitters and/or receivers is typically an important metric to predict the performance of a given wireless network. A review of the sparse literature shows that there are generally two judicious tools to evaluate the amount of regularity in a point pattern. i) The coefficient of variation $(\mathrm{CoV})$ metrics, introduced for the first time in [121]. They are constructed based on specific geometrical characteristics of point patterns, such as the area of Voronoi cells, the length of Delaunay triangulation edges, and the nearest neighbor function. $\mathrm{CoV}$ metrics are typically normalized by a given constant [122] such that their value in the context of the PPP equals 1 . Valuable applications can be found in [102], [118][120]. ii) The average deployment gain introduced in [67, Equation (11)] to measure the minimum horizontal gap
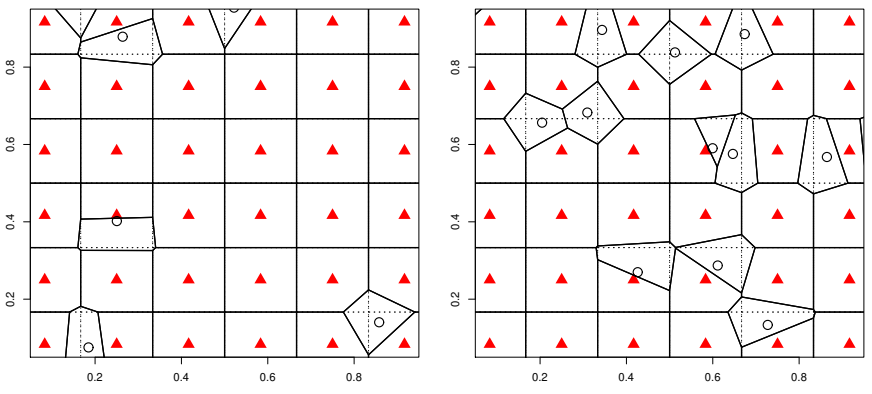

Fig. 5. Two realizations of the proposed PP constructed as a superposition of a stationary perfect lattice (red triangles) and a PPP (black circles).

in terms of the mean square deviation between the two curves of the SIR distribution under the PPP and the point pattern under investigation.

- Combination of PPP and stationary grid: Due to the observation that all the nodes of a PL are subject to random perturbation, the authors of [66] confirmed via experiments on realistic deployments that PLs cannot accurately capture spatial dependence between nodes. Alternatively, the authors of [123] proposed a new approach to capture soft repulsion between nodes as a combination of two extreme sub-structures, namely a totally random PP (i.e., PPP) and a stationary deterministic lattice. That is, the repulsiveness of the outcome PP is softly tuned based on the ratio between the densities of the PPP and the stationary grid (see Fig. 5).

4) Aggregative point processes: There are several aspects exhibiting clustering in realistic wireless networks. For instance, there will be a tendency towards clustering for indoor transmitters covering building's interior, or transmitters serving clustered users around hotspots, or even vehicles clustered due to traffic and intersections. Also, UEs of a D2D communication network need to lie in close proximity of each other, and sometimes, the clustering of nodes may be logically induced by some MAC protocols [124]-[129]. In such circumstances of geometrically and logically induced clustering, aggregative PPs are required for an accurate evaluation of networks performance. A common way to capture the clustering of nodes is by considering a further generalization of the PPP via the IPPP, where the distance-dependent density of the IPPP increases in regions of interest. However, one main shortcoming of the IPPP is its non-stationarity, which limits the use of tractable simplifications considered in the case of stationary PPs and also ceases the concept of the typical user where the performance evaluation becomes dependent on the location of the user under investigation. In the following, we review the key aggregative PPs used in the literature to overcome such limitation.

- Cox cluster point process: A further generalization of the finite ${ }^{9}$ IPPP is the stationary finite Cox process constructed by randomizing the parameters of the IPPP model. Typically, the intensity of the IPPP becomes a random variable mapped with realizations of a stationary

\footnotetext{
${ }^{9}$ Stationarity or homogeneity of a PP implies implicitly infinite point patterns. In other words, it is realistic to consider large inhomogeneous point patterns as part of a stationary PP while IPPP is generally a finite PP [14].
} 
random field with positive values (i.e., the intensity field). The Cox PP is also known as a doubly stochastic PP since its construction is tracked on two steps: i) Generate realizations of the random field $\{\Lambda(y)\}$, i.e., parent points; and next, ii) conditioned on a realization $\Lambda(y)$ of the random field, point pattern is generated with an IPPP of density $\lambda(y)=\Lambda(y)$, i.e., daughter points, where parent points are not observable and do not form part of the resulted point pattern. Depending on the construction method of the random field $\{\Lambda(y)\}$, several flexible families of the Cox PP may be established. For instance, the log-Gaussian Cox process (LGCP) is considered when the logarithm of the random field is a real-valued Gaussian process. In other words, the clustering of point patterns in LGCP may be smoothly adjusted by acting on the mean and variance of the distribution, where a zero variance is equivalent to the PPP case, and an increasing variance (with constant mean) is equivalent to an increasing tendency towards clustering. Another interesting doubly stochastic PP is the $\alpha$-stable Cox PP [130], [131], in which the random field follows the $\alpha$-stable distribution. The shot-noise Cox PP [132] is obtained by generating the random field by a general PP $\Psi_{\mathrm{p}}$, where at each parent point $x \in \Psi_{\mathrm{p}}$, the daughter points $\Psi_{\mathrm{d}}$ are generated by an IPPP with density $m_{x} \psi(y-x)$, where $m_{x}$ is the average number of points clustered around $x$, and $\psi($.$) is the$ PDF of the distance between a daughter point $y$ of the cluster and $x$. The density of the outcome Cox PP is then expressed as

$$
\lambda(y)=\sum_{x \in \Psi_{p}} m_{x} \psi(y-x), \quad \forall y \in \Psi_{\mathrm{d}} .
$$

The Cox PP is typically investigated in VANETs [82], [133], [134], where a doubly stochastic process is useful to capture the randomness of roads (modeled by a Poisson line process (PLP)) as well as that of nodes location (modeled by a 1D PPP) (see Fig. 6). Alternatively, and based on empirical data of realistic networks, the authors of [131] observed that user-centric capacity-driven behavior of modern BS deployments is accurately captured by heavy-tailed distributions of the BS density, particularly the $\alpha$-stable distribution. In [130], analytical investigation in addition to empirical data fitting is obtained by considering a generalized PPP setup with $\alpha$-stable distributed BS density. In [135], the spatial clustering degree of users (i.e., level of heterogeneity) is captured via the LGCP, where it is observed that the network performance decreases when users are clustered without being correlated to BSs location.

- Poisson cluster process (PCP): In Cox cluster PP, the number of parent points follows a general PP while that of daughter points follows a PPP. The PCP, however, is based on a reciprocal approach where the number of parent points follows a PPP while that of daughter points follows a general PP. A representative family is the Gauss-Poisson PP, in which daughter points are either no points, one, or two points, with respective probabilities $p_{0}, p_{1}$, and $p_{2}=1-p_{0}-p_{1}[6]$. Another special case

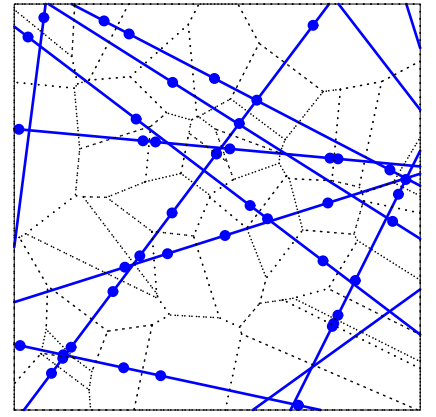

(a)

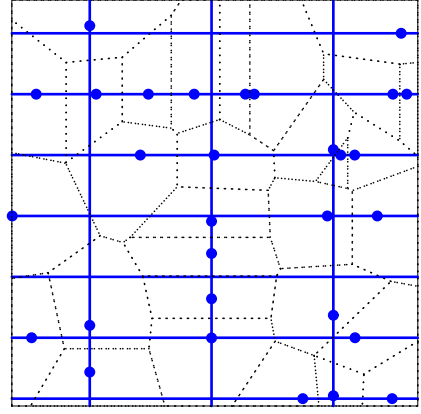

(b)
Fig. 6. Two realizations of roads (solid lines) modeled by a Poisson line process (a) and by a deterministic set of lines (b). Vehicles (dots) are modeled by a 1D PPP with similar densities in (a) and (b). Voronoi tessellation (dotted lines) reflects the association region of each vehicle.

of the PCP is when daughter points are scattered i.i.d. around the origin and their number is Poisson distributed, which yields the family of the Neyman-Scott PP, also considered as a special case of the shot-noise Cox PP. In this way, considering the parent PP as HPPP and based on the expression of $\psi($.$) in (26), two important models of$ the Neyman-Scott PP are commonly constructed, namely the Matérn cluster processes (MCP), where daughter points are i.u.d in a ball $b(x, \delta)$ centered at each parent point $x \in \mathbb{R}^{d}$, and Thomas cluster processes (TCP), where daughter points are symmetric normal distributed. Based on simulations and model fitting, relevant works in the literature compared empirical data of existing shared networks [136] and vehicular networks [137] with the accurate PP from MCP, TCP, and LGCP. Results showed that the LGCP is the most suitable PP to characterize point patterns that exhibit strong tendency towards clustering, while analytical flexibility is in favor of the others, as performed in [124]-[129] for MCP and TCP, and in [138], [139] for the Gauss-Poisson PP.

- The conditional thinning approach: A tractable generative approach to capture the tendency of nodes towards clustering (i.e., reduced homogeneity) is by considering a specific independent thinning. Typically, the authors of [140], [141] introduced a specific thinning of retention probability $p$ conditioned on the serving BS and complementarily dependent on the empty probability of other BSs [142] (i.e., the probability that a BS does not serve any user). A value of $p=1$ is equivalent to a uniform distribution of users, while a decreasing value of $p$ is equivalent to clustering of users around the serving BS.

5) Wide versatile point processes: Despite diversity of the previous discussed PPs, they are restricted to capture point patterns that exhibit either repulsion or clustering. However, in realistic deployments, we usually find a combination of repulsion and aggregation at different levels, and hence a compelling need for more general PPs. In the following, we consider a third type of PPs, namely the wide versatile PPs, that with regard to their typical construction may capture both repulsion and clustering.

- Geyer saturation point process (GSPP): The first PP is the GSPP seen as a natural generalization of the 


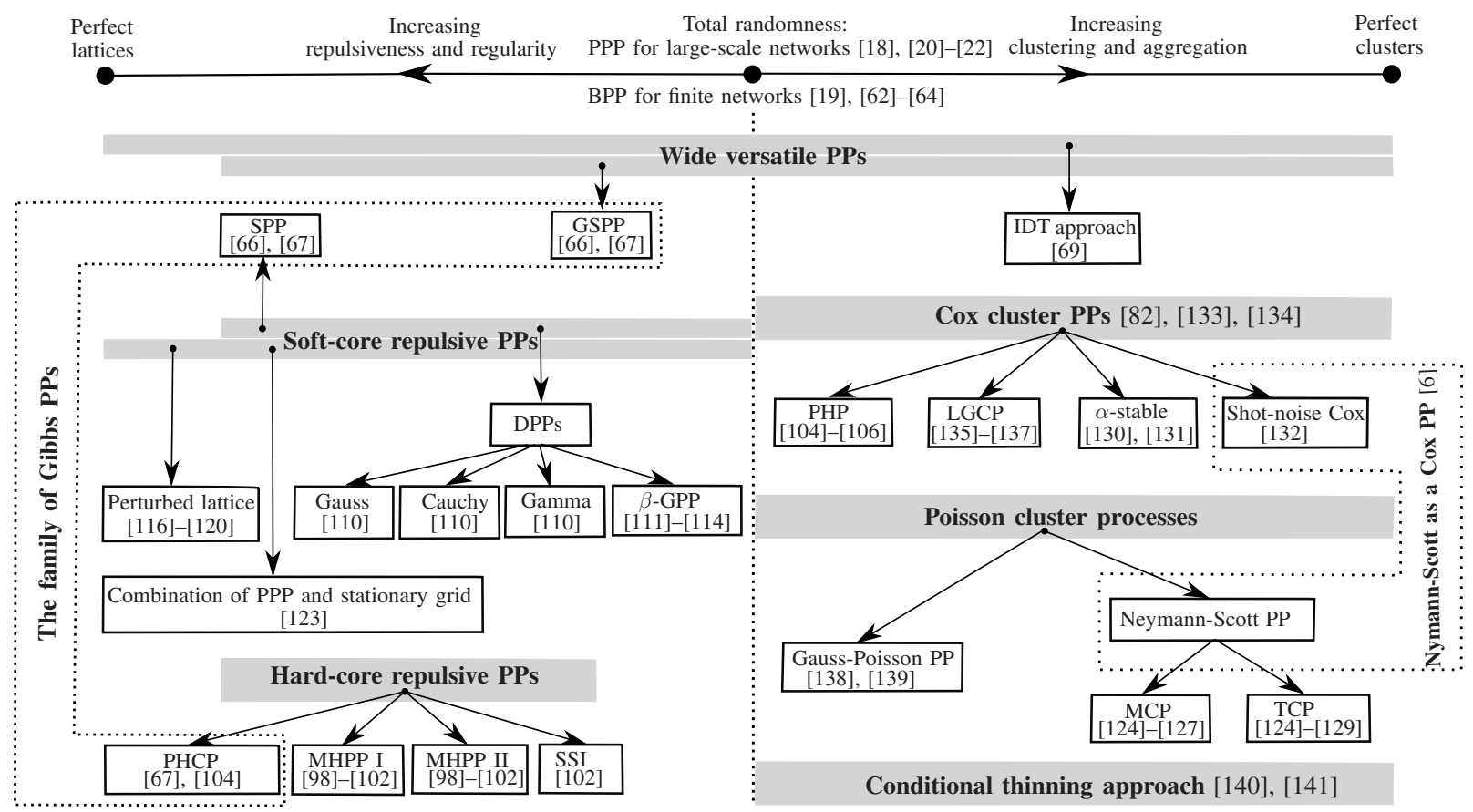

Fig. 7. Taxonomy of PPs and frameworks used to model nodes in wireless communication networks.

SPP. Actually, when $\gamma$ in (22) is below 1, the GSPP is equivalent to an SPP and then captures repulsiveness. However, in the case of clustering (i.e., $\gamma>1$ ), the multivariate PDF in (22) is not integrable for $s(u) \rightarrow \infty$. To overcome this, the GSPP is then saturated as

$$
\varrho^{(n)}(u)=\kappa \beta^{n(u)} \gamma^{\min (s(u), t)},
$$

where $t$ is a constant to bound the trend of $s(u)$. If $t$ is large enough, the GSPP can capture both repulsion and clustering depending on the fluctuation of $\gamma$. Moreover, if $t=0$ or $\gamma=1$, the GSPP is equivalent to a PPP.

- The inhomogeneous double thinning (IDT) approach: The second PP is an analytical framework, namely the inhomogeneous double thinning (IDT) approach, introduced in [69] in such a way to capture the interaction degree between points based on the superposition of two conditionally independent IPPPs. In fact, by conditioning on the serving BS, the first IPPP captures the fluctuation degree of the distance between the typical user and the serving BS (via the $F$-function), while the second IPPP captures the fluctuation degree of the distance between the typical user and interfering BSs (via Ripley's $K$ function). Interestingly, based on two triplets of parameters (i.e., one triplet for the $F$-function and the other triplet for the $K$-function), it is observed that the IDT model can accurately fit the structure of a wide range of wireless networks, where nodes location can exhibit spatial repulsion and/or clustering.

Fig. 7 illustrates a comprehensive taxonomy of the PPs used in the literature of wireless networks modeling and analysis. Typically, the gray bar reflects the range of variation in the degree of interaction of each PP family. For instance, hard-core PPs can only reflect structures with hard-core repulsion dis- tance, without being able to capture totally random structures (PPP or BPP) or perfect lattices. DPPs which are part of softcore PPs, can capture structures ranging from the PPP to some repulsive structures below perfect lattices. However, other softcore PPs such as perturbed lattice and the combination of a PPP and a stationary grid can model more point patterns ranging from PPP to perfect lattices. Interestingly, the IDT approach can model structures ranging from the two extremes.

Table III classifies the PPs, used in the SG literature for modeling and analysis of wireless networks, by modeling use cases and various degrees of tractability. An important key measure of interest is the ability of the PP to permit the derivation of the PGFL of the interference at a given arbitrary point, which in turn allows to derive various performance metrics (e.g. coverage probability, ergodic rate). Three classes of PPs are identified; those enabling to derive the interference's PGFL, those failing to derive it so that an approximation of the PGFL or the mean value of the interference is made, and those with unknown PGFL and mean value of the interference.

\section{B. The Superiority of the Poisson Point Process}

With a comprehensive exploration of all the abovementioned references related to proposals for modeling wireless networks with PPs beyond the PPP, it is almost straightforward to infer that such PPs are more accurate than the PPP for modeling emerging wireless architectures. However, they are mathematically less tractable to derive the contact distance function, the nearest neighbor function, and hence we can only approximate the interference and performance behavior. Also, modeling node locations with the previous PPs does not provide a significant change to system design insights as compared to the PPP case [6], [26], [47], [69]. 
TABLE III

TAXONOMY OF PPS IN TERMS OF THE ABILITY TO CHARACTERIZE THE INTERFERENCE AND ANALYTICAL TRACTABILITY

\begin{tabular}{|c|c|c|c|c|c|c|c|c|}
\hline & \multicolumn{4}{|c|}{ Modeling use cases } & \multirow[b]{2}{*}{ Interference characterisation } & \multirow[b]{2}{*}{ References } & \multirow[b]{2}{*}{ Analytical tractability } & \multirow[b]{2}{*}{ Comments } \\
\hline Point process & Regularity & Repulsion & Clustering & Independence & & & & \\
\hline Stationary regular lattice & - & & & & Exact mean & [47], [91] & High & $\begin{array}{l}\text { - More efficient to design good channel access schemes } \\
\text { as compared to networks where node locations are perceived } \\
\text { as random or in motion. } \\
\text { - System-level performance evaluation requires complex and } \\
\text { time-consuming Monte Carlo simulations. } \\
\text { - Do not capture the geometry of Hetnets. }\end{array}$ \\
\hline PPP & & & & - & Exact PGFL & {$[18]$} & High & $\begin{array}{l}\text { - Enhanced tractability and mathematical convenience, more } \\
\text { simplifications compared to other PPS, captures randomness } \\
\text { of network geometry. } \\
\text { - Cannot capture the geometry of realistic scenarios, where } \\
\text { nodes are highly correlated (repulsion or clustering). }\end{array}$ \\
\hline BPP & & & & - & Exact PGFL & [63] & Medium & $\begin{array}{l}\text { - The PPP version to model finite networks. } \\
\text { - Reduced tractability as compared to the PPP. }\end{array}$ \\
\hline МНСРР & & - & & & Approximate mean & [98], [99] & Low & $\begin{array}{l}\text { - Capture the mutual repulsion between nodes. } \\
\text { - Underestimates the density of transmitters in high density } \\
\text { of the parant PPP points, which affects the interference } \\
\text { estimation. }\end{array}$ \\
\hline SSI & & & & & Unknown & {$[102]$} & Unknown & $\begin{array}{l}\text { Overcomes the limitations of MHCPP in terms of } \\
\text { underestimating the density of transmitters in high } \\
\text { density of the parent PPP points. }\end{array}$ \\
\hline PHCP & & - & & & Exact PGFL & {$[104]$} & Low & \multirow{3}{*}{$\begin{array}{l}\text { - Easy interpretation of the model and flexible simulations. } \\
\text { - GSPPP: Suitable for a wide range of PPs ranging from the } \\
\text { aggregative family to the repulsion one. } \\
\text {-No closed form expressions for the moments. } \\
\text { - Can resort to time consuming simulations. }\end{array}$} \\
\hline SPP & & - & & & Unknown & [66], [67] & Unknown & \\
\hline GSPP & & $\bullet$ & - & - & Unknown & [66] & Unknown & \\
\hline PHP & & & - & & Approximate PGFL & {$[105],[106],[108]$} & Medium & $\begin{array}{l}\text { Enables to conceptualize hard-core repulsion between points } \\
\text { based on the tractability of two independent HPPSs. }\end{array}$ \\
\hline DPP & & - & & & Exact PGFL, Exact mean & {$[109]-[114]$} & Low & $\begin{array}{l}\text { - The moments are known as they are described by certain } \\
\text { determinants of matrices } \\
\text { - Involving analysis of the contact distribution function and } \\
\text { the SINR's distribution }\end{array}$ \\
\hline Perturbed lattice & - & - & & - & Approximate mean & {$[116],[117]$} & Medium & $\begin{array}{l}\text { - Enables to softly capture point patterns that exhibit perfect } \\
\text { regularity. } \\
\text { - All nodes of a PL are subject to random perturbation, which } \\
\text { reduces the accuracy to capture spatial dependence between } \\
\text { nodes in realistic deployments. }\end{array}$ \\
\hline $\begin{array}{l}\text { Superposition of PPP } \\
\text { and Shiffed lattice }\end{array}$ & - & - & & - & Approximate PGFL & [123] & Medium & $\begin{array}{l}\text { - Overcomes the limitations of the perturbed PL. } \\
\text { - Involving analysis of the SINR's distribution. }\end{array}$ \\
\hline Alpha-stable Cox & & & - & & Exact PGFL & [130] & Low & $\begin{array}{l}\text { - Superior accuracy to statistically model the varying BS } \\
\text { density in different areas. } \\
\text { - Involving analysis of the SINR's distribution inducing } \\
\text { reduced computational efficiency. }\end{array}$ \\
\hline LGCP & & & - & - & Unknown & {$[135]-[137]$} & Unknown & $\begin{array}{l}\text { - Its construction is based on elegant simplicity as the random } \\
\text { field is a real-valued Gaussian process. } \\
\text { - Can serve as a universal model to fit realistic multi-network } \\
\text { empirical data }\end{array}$ \\
\hline РСР & & & - & & Exact PGFL & [125]-[128], [138], [139] & Medium & $\begin{array}{l}\text { Enables to capture spatial coupling between user and BS } \\
\text { locations, which is in line with the } 3 \mathrm{GPP} \text { simulation models. }\end{array}$ \\
\hline $\begin{array}{l}\text { Conditional thinning } \\
\text { approach }\end{array}$ & & & • & - & Exact PGFL & [140], [141] & High & $\begin{array}{l}\text { Captures the tendency of users towards clustering } \\
\text { (i.e., reduced homogeneity) around the serving BS. }\end{array}$ \\
\hline IDT approach & - & - & - & - & Exact PGFL & [69] & Medium & $\begin{array}{l}\text { Captures the interaction degree between points based on the } \\
\text { superposition of two conditionally independent IPPPs }\end{array}$ \\
\hline
\end{tabular}

Accordingly, in some analytical contexts, it is generally more appropriate to favor mathematical tractability with physically meaningful insights on system design, rather than increasing modeling accuracy but with a huge loss on tractability and mathematical flexibility. In other words, the tractability of the PPP sometimes justifies its possible inaccuracy. Subsequently, we consider four recent results that endorse even the accuracy of the PPP relatively to other beyond-PPPs:

- In [55, Theorem 3], the authors support analytically the assumption that modeling node locations through PPP is a realistic hypothesis since a given general model for a large structure of node locations can be seen under the effect of sufficiently strong log-normal shadowing, i.e., greater than approximately $10 \mathrm{~dB}$, as equivalent to the PPP model. In other words, instead of modeling node locations with a given general PP under log-normal shadowing, we can equivalently consider a perturbation of node locations, which may lead to a totally random structure depending on the intensity of shadowing.

- In [143]-[145], the authors showed that the slope of the SIR distribution is the same for almost all motion- invariant PPs, i.e., the SIR distribution of a given network model is a shifted version of the other network models. For example, the horizontal gap between the PPP and the triangular lattice is approximately a constant of $3.4 \mathrm{~dB}$ for a wide range of SIR regimes. Interestingly, instead of modeling point patterns of a given network by a lesstractable but more accurate PP, one can use the PPP, i.e., the reference network model, endowed with its enhanced tractability and add some weight to the outcome network performance being evaluated under the PPP assumption.

- In [140], [141], the authors explored the PPP under a tractable generative model, namely the conditional thinning approach, which allows capturing smoothly a wide range of clustered UEs ordered from a totally random structure to a very clustered one. Consequently, the PPP can be harnessed in a meaningful way to create new PPs that are endowed with similar tractability as the PPP but can also capture inter-points interactions.

- In [69], and since the IPPP is the most tractable alternative to the PPP, the authors introduced the IDT approach that can be used as the most tractable version of the PPP, and fully able to capture a wide range of network models 


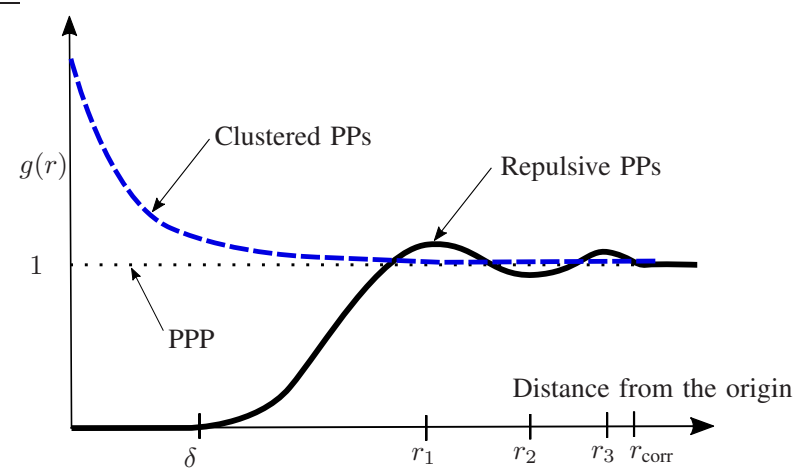

Fig. 8. Typical shape of pair correlation function for the three classes of PPs.

from clustered PPs to stationary deterministic lattices.

Since SG is also endowed with an important statistical theory [14], the PPP is typically leveraged as a reference PP to build statistical tools that enable to characterize the class of a given PP (totally random, clustered, or repulsive) or even to compare between PPs. Subsequently, we will review the key statistical methods used in the literature of wireless networks modeling and analysis to characterize PPs or fit them to realistic empirical data.

\section{SG STATISTICAL ANALYSIS}

Several tools are used in the theory of PPs and spatial statistics to detect deviations from the PPP and characterize the interaction between points, particularly in terms of type, strength, and range [1], [6], [14]. These tools are also used as fitting methodologies to identify an appropriate PP model for some empirical data, [66], [67], [90], [113].

\section{A. Comparison between Point Processes}

Using the observation that the contact function and the nearest neighbor function are identical in the PPP, the $J$ function is introduced in spatial statistics for $r>0$ as

$$
J_{y}(r)=\frac{1-G_{y}(r)}{1-F_{y}(r)} \text {. }
$$

That is, $J(r)=1$ in the case of a PPP. In clustered PPs, an arbitrary point of the plane is likely to be farther away from a given point of the PP rather than in the context of the PPP, whereas in the other direction, clustered points tend to lie closer to their nearest neighbors, hence $F_{y}(r)<G_{y}(r)$ and then $J_{y}(r)<1$. Similarly, $J_{y}(r)$ is greater than 1 in the case of repulsive PPs. However, it is possible to construct sometimes a non-Poisson PP that checks $J(r)=1$, which reduces the accuracy of the $J$-function in characterizing PPs [14].

An alternative approach is by considering second order summary characteristics such as the pair correlation function [123], defined for a PP $\Psi \subset \mathbb{R}^{d}, \forall x, y \in \Psi$ as

$$
g(x, y)=\frac{\varrho^{(2)}(x, y)}{\varrho^{(1)}(x) \varrho^{(1)}(y)},
$$

where $\varrho^{(1)}($.$) and \varrho^{(2)}($.$) are, respectively, the first and second$ moment densities of $\Psi$.

When $\Psi$ is isotropic, $g(x, y)$ is only dependent on the distance $r$ between points $x$ and $y$ but not on their locations.
That is, for a completely random PP (i.e., the PPP), $x$ and $y$ are totally independent and then $g(r)=1$. In clustered PPs, $\varrho^{(2)}(x, y)$ is likely to overcome $\varrho^{(1)}(x) \varrho^{(1)}(y)$, then $g(r)>1$ for small $r$ and converges to 1 as $r$ increases. For hard-core repulsive PPs, where inter-points distance is almost greater than a certain barrier distance $\delta$, the pair correlation function equals to 0 when $r<\delta$. As $r$ becomes greater than $\delta, g(r)$ can exceed 1 and fluctuates around it with increasing $r$.

Fig. 8 describes the overall trend of $g(r)$ as a function of the PP class. Typically, $r_{\text {corr }}$ is the distance describing the approximate size of clusters. $r_{1}$ is the distance to the closest neighbors with most frequent short inter-point distance. $r_{2}$ is the distance at which $g(r)$ contacts its first minimum after $r_{1}$, and can be interpreted as the distance to regions with a small number of points beyond the nearest neighbors. $r_{3}$ is the second maximum of $g(r)$, interpreted as the distance to the regions with further neighbors [14].

Other popular second order summary statistics used to capture inter-points correlation are Ripley's $K$ and $L$-functions defined for a stationary PP of density $\lambda$ and $r \geq 0$ as

$$
K(r)=\frac{\mathbb{E}_{\mathrm{o}}^{!}(\Psi(b(o, r)))}{\lambda} \text { and } L(r)=\sqrt{\frac{K(r)}{\pi}} .
$$

We note that $K(r)=\pi r^{2}$ and $L(r)=r$ in the case of a PPP. Repulsive and clustered PPs are however, respectively, characterized by smaller and larger $K$ and $L$-functions as compared to the PPP. More discussions about PPs statistics and nodes real deployment characterization can be found in [14], [45], [66], [67], [69], [90], [95], [96], [113].

Characterizing PPs based on their summary statistics is generally not sufficient to study the impact of inter-point interaction on macroscopic properties [14], [66], [67]. Interestingly, the authors of [146], [147] developed PP ordering based on the directionally convex (dcx) order, where for two given realvalued PPs $\Psi$ and $\Phi$ of the same dimension, $\Psi$ is said to be less than $\Phi$ in dcx, if and only if for all directionally convex ${ }^{10}$ function $f$ on $\mathbb{R}^{d}, \mathbb{E}(f(\Psi)) \leq \mathbb{E}(f(\Phi))<\infty$ and we denote $\Psi(.) \leq_{\mathrm{dcx}} \Phi($.$) . Typically, it has been shown in [146,$ Proposition 3.4 and Corollary 3.1] that the dcx order cover PPs comparison based on the pair-correlation and $K$-functions, where the largest PP in terms of dcx order is generally the one with the greatest pair-correlation and $K$-function, assuming the same mean number of points in the observation window. That is, the PPP is also taken as the reference PP on dcxbased comparison, where repulsive and clustered PPs are, respectively, smaller and larger in dex order as compared to the PPP. They are then referred to as sub and super-Poisson, respectively.

In general, sub- and super-poissonianity can occur simultaneously but at different spatial scales, e.g., clustering at large scales and regularity at small scales. As an illustration, using the spatstat package in the $\mathbf{R}$ language, Fig. 9 shows the estimation of summary statistics $J(r), g(r)$, and $K(r)$ from a homogeneous PPP generated in the window $W=[0,10] \times[0,10]$. We can see in particular the fluctuation of summary statistics with the range of observation.

\footnotetext{
${ }^{10}$ For any $x, y, z$ and $u \in \mathbb{R}^{d}$, such as $x \leq y, z \leq u$ and $y+z=x+u$, we have $f(y)+f(z) \leq f(x)+f(u)$.
} 
Two HPPP realizations with $\lambda=0.3$ and 0.7
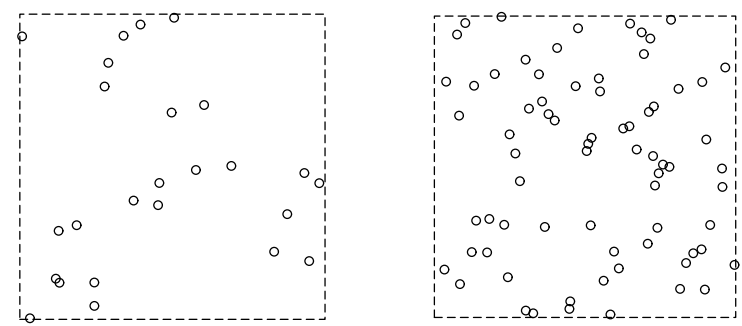

The kernel density estimate of the two PPP's realizations
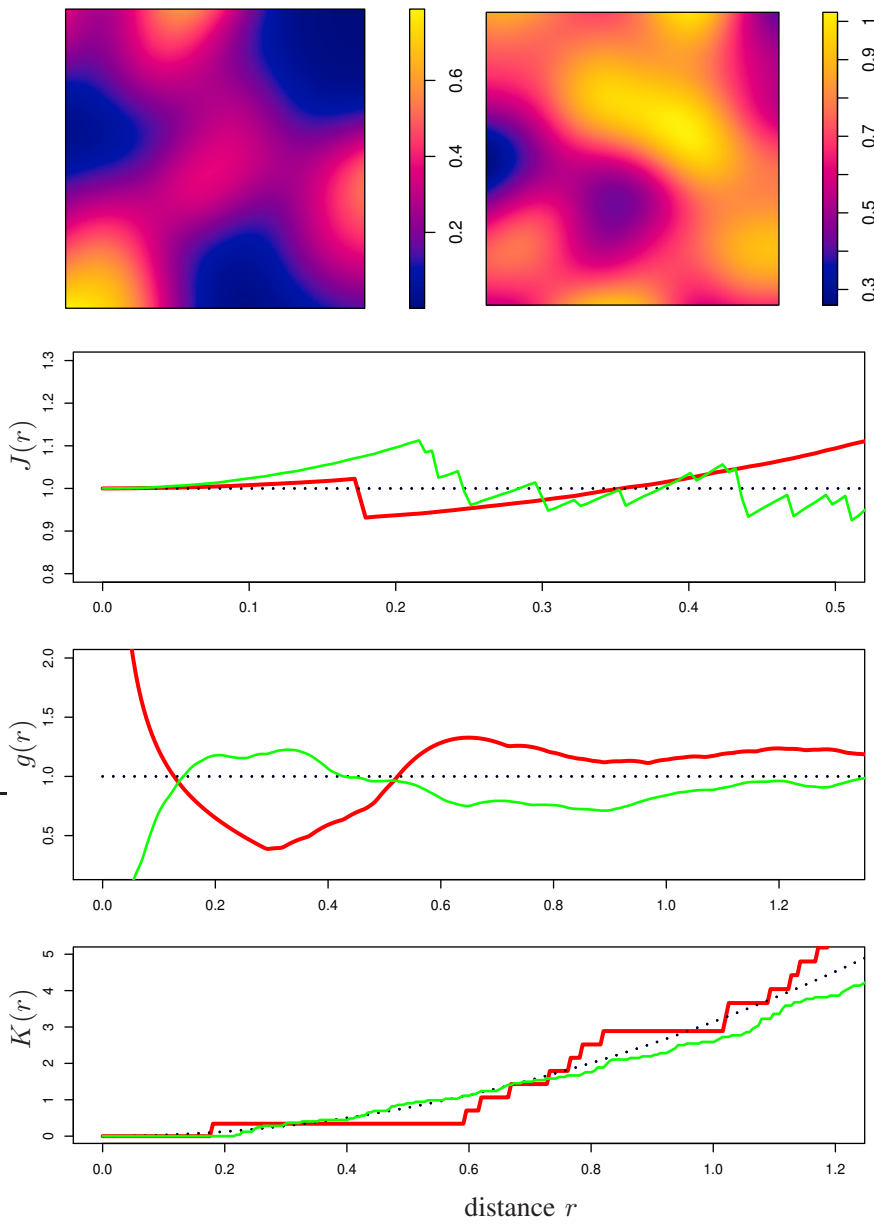

Fig. 9. Estimation of summary statistics $J(r), g(r)$, and $K(r)$ from two HPPP in $W=[0,10] \times[0,10]$. The red bold line is for HPPP density $\lambda=0.3$, the green thin line is for $\lambda=0.7$, and the dotted lines are the theoretical values of the summary statistics assuming an infinite expansion of the window $W$

\section{B. Modeling Real Nodes Deployment}

In the following, we will review the statistical methods used to fit several PP candidates to empirical data of realistic networks. Next, we will investigate the various metrics explored in the literature to select the best fitted PP model, i.e., the goodness-of-fit.

1) Fitting the structure of nodes: In classical statistics, the likelihood function describes the probability of observing data samples given some model parameter $\theta$ [14], [68]. Similarly, in the context of SG, the parameters of the PP model are approximated from existing point pattern $\mathbf{x}=\left\{x_{1}, \ldots, x_{n}\right\}$, where the likelihood function is maximized, yielding to param- eter estimation that best fits the data samples, e.g., the ratio between the number of point patterns and the window area is a natural estimator for the parameter density of an HPPP, the hard-core distance in inhibitive PPs is simply estimated by the minimum inter-point distance in the empirical data, etc.. The general formulation for the likelihood function of three classes of representative PPs, namely the HPPP, the IPPP, and finite Gibbs PP, can be found in [68]. However, due to the lack of closed-form expressions for the normalizing function rendering the likelihood function a PDF, the maximization problem for the likelihood function when considering several PPs beyond the PPP, is generally intractable [14], [68]. To overcome such limitation, the pseudo-likelihood function of a given PP is defined in terms of the conditional intensity at a given point of the sample pattern [68, Equations (6), (7)]. Also, when the conditional intensity is not available or parameter estimators are of reduced accuracy, which is typically the case for aggregative PPs, the minimum contrast method is proposed. In this way, the key idea is to define PP parameters that minimize the gap between the summary statistic of this PP and the estimated one from empirical data. This gap, as a function of the PP model parameters $\theta$, is typically expressed as

$$
\Delta(\theta)=\int_{s_{1}}^{s_{2}}\left|\widehat{S}^{m}(r)-S_{\theta}^{m}(r)\right|^{n} \mathrm{~d} r
$$

where $\widehat{S}(r)$ is the estimated summary statistic from empirical data over a range radius $s_{1} \leq r \leq s_{2}$ and $m, n>0$ are parameters in the method.

2) Metrics for the goodness-of-fit: After the fitting procedure of several PP candidate models to the empirical data, comes the goodness-of-fit phase where the best fitted PP to empirical data is selected. In the following, we outline the key techniques used in the literature for the goodness-of-fit procedure:

- Summary statistics simulated envelope test (3SET): The most common approach for hypothesis testing is by evaluating the gap between summary statistics curves of the empirical data and the fitted PP model. In fact, by simulating the summary statistics of the fitted PP model, we end up getting the lower and upper envelopes that reflect the confidence interval. Next, the fitted PP model is considered as a good model if the curve of the estimated summary statistic of the empirical data, falls into the envelope with increased probability. Otherwise, the PP model may be rejected based on the 3SET method. Typically, the $K$ and $L$-functions are the most popular summary statistics considered for the 3SET method [66], [67], [110], [113]. However, other summary statistics such as $G, F$, and $J$-functions are investigated in [110], [113], [148]. Besides, if the curve of the estimated summary statistic of the empirical data lies within the envelope of several fitted PP models, a specific quantity may be considered to select the most suitable PP model, namely the root mean square deviation (RMSD), defined 
from (31) as

$$
\mathrm{RMSD}=\sqrt{\frac{1}{N} \sum_{k=1}^{N}\left(\widehat{S}\left(r_{i}\right)-S_{\theta}\left(r_{i}\right)\right)^{2}},
$$

where $N$ is the number of samples.

- SINR distribution: Since the SINR distribution is tightly related to the network geometry (i.e., inter-points interaction), it is used as an evaluation metric to select the most suitable PP model for empirical data. Assuming that the reference user $y$ is located at the origin of the point pattern $\Psi$ and connected to the nearest point $x_{0} \in \Psi$, the downlink SINR is defined as

$$
\operatorname{SINR}\left(x_{0} ; y\right)=\frac{P_{\mathrm{tx}} h_{x_{0}} \ell\left(\left\|x_{0}\right\|\right)}{\sigma^{2}+\sum_{x_{i} \in \Phi \backslash\left\{x_{0}\right\}} P_{\mathrm{tx}} h_{x_{i}} \ell\left(\left\|x_{i}\right\|\right)},
$$

where $P_{\mathrm{tx}}$ is the BS transmit power, $\ell($.$) is the path loss$ function, $h_{x}$ is a random variable that captures multipath fading and/or shadowing between user $y$ and BS $x$, and $\sigma^{2}$ is the variance of noise power.

Based on simulations, the SINR distribution of the empirical data and the fitted PP models are evaluated w.r.t. a threshold in $\mathrm{dB}$, then the suitable PP model is selected using envelope matching and eventually the RMSD method [66], [67], [117], [149].

- Geometry-based evaluation metrics (GBEM): Two main geometry characteristics are considered in the literature as higher-order properties in PP model selection, namely the Voronoi area distribution (VAD) [2], [66], [122], [150] and the Delaunay triangulation edges length distribution (DTELD) [102], [118]-[120]. The use of VAD and its dual DTELD is actually justified given the observation that coverage regions of BSs in a cellular network generally converge to Voronoi cells [4, Proposition 5.5.11], where the VAD is commonly approximated in the case of a PPP by a generalized gamma function [122].

\section{StOchastic GEOMETRY FOR MODELING AND ANALYSIS OF WIRELESS NETWORKS}

Modeling wireless networks is commonly considered as a set of conceptual choices to study a real or an imaginary communication scenario. Such model preferences are typically related to i) network elements, e.g., location model (deterministic, random, or mobile), node type (transmitter, receiver, or both), ii) their attributes, e.g, transmit power and antenna types, iii) the environment characteristics in which they operate, e.g, propagation effects, and iv) the interplay properties between nodes, e.g., association policy, coordination, and spatial interaction. In some cases, we can also include analytical and experimental tools used in the study, in addition to the considered key performance metrics. In the following, we will review the plethora of modeling choices made in the literature of SG-based modeling and analysis.

\section{A. Modeling Network Elements}

Based on the SG approach, elements of a wireless network are deemed to be hierarchically modeled in such a way that subscribers are 0-level stations, BSs are 1-level stations directly connected to 0-level stations, switching centers are 2level stations directly connected to BSs, and so on [2], [142]. Besides, depending on the system model being considered, network elements can be partially or entirely distributed according to particular PPs and receiver/transmitter locations can be correlated ${ }^{11}$ or not, e.g., coupling of users and BSs location in a user-centric capacity-driven cell deployment. Table IV summarizes the state-of-the-art main configurations used for modeling the location of users and BSs.

\section{B. Modeling Propagation Effects}

In a wireless network composed of many spatially dispersed nodes, communication is typically impaired by various deficiencies like wireless propagation effects introduced by i) the attenuation of radiated signals with blockages (shadowing), ii) receiving multiple copies of the same transmitted signal (multipath fading), and iii) signal losses with distance (path loss). In general, the received power at the typical receiver located at a distance $r$ from the transmitter, is expressed as

$$
P_{\mathrm{rx}}=P_{\mathrm{tx}} \ell(r) \prod_{k} Z_{k}
$$

where $P_{\mathrm{tx}}$ is the reference transmitted power, $\ell($.$) is the path$ loss function, and $\left\{Z_{k}\right\}$ are independent random variables accounting for propagation effects.

It is worth mentioning that the effect of shadowing is generally captured via log-normal distributed random variables where key parameters are fitted from field measurements. However, in view of the analytical intractability of such distribution PDF, a common approach in SG-based frameworks is to absorb shadowing model into the intensity function of a new PPP by means of the displacement theorem. Representative examples can be found in [16], [26], [55], [58], [86], [88], [89], [164], [165]. Also, modeling shadowing via random variables fails to reflect the distance-dependence of blockage effects given that shadowing intensity needs to naturally grow with increased transmit-receive distance, particularly in higher-frequency bands where signals are more vulnerable to blockages. That is, the authors of [166] proposed to capture blockages effect via the product $\prod_{i=1}^{M} \gamma_{i}$, where $0 \leq \gamma_{i} \leq 1$ is the ratio of power loss due to the $i$ th blockage, and $M$ is the random number of blockages intersecting the transmitreceive link. Using tools from random shape theory [166], $M$ is shown to follow a Poisson distribution with parameter dependent on the blockages density, the link distance, and the average dimensions of blockages.

Regarding path loss functions, Table V summarizes the key models used in the literature of SG-based modeling and analysis. Typically, the great majority of works consider the simplistic single slope unbounded path loss model (UPM) (model \#1 in Table V) given its ability to derive reliable and tractable results especially for sparse networks wherein the

\footnotetext{
${ }^{11}$ This term should not be confused with temporal and spatial correlation in BS activity factors where the former is induced by the mobility of receivers across neighboring transmitters, while the latter is induced by correlation through interference and load traffic between neighboring transmitters [151].
} 
TABLE IV

TYPICAL CONFIGURATION OF BSS AND USERS IN A STOCHASTIC GEOMETRY BASED MODELING

\begin{tabular}{|c|c|c|c|c|c|}
\hline \multirow{2}{*}{ Configuration of BSs } & \multirow{2}{*}{ Configuration of UEs } & \multirow{2}{*}{ UE-BS coupling? } & \multicolumn{2}{|c|}{ Adopted PP } & \multirow{2}{*}{ Comments } \\
\hline & & & For BSs & For UEs & \\
\hline Uniformly random & Uniformly random & No & PPP & PPP & $\begin{array}{l}\text { Total independence between UEs and BSs } \\
{[18],[20],[21] .}\end{array}$ \\
\hline Uniformly random & Uniformly random & Yes & PPP & PPP & $\begin{array}{l}\text { UE-BS coupling can be captured through } \\
\text { i) a specific dependent thinning as in [140] } \\
\text { [141], or via ii) a power control scheme } \\
\text { in uplink networks as in [22], [152]-[159]. }\end{array}$ \\
\hline Uniformly random & Uniformly random & Yes & BPP & BPP & $\begin{array}{l}\text { Correlation between UEs and BSs is } \\
\text { introduced via the finite number of BSs } \\
\text { and UEs in a given area [19], [62]-[64]. }\end{array}$ \\
\hline Uniformly random & Clustered & Yes & PPP & $\mathrm{MCP}, \mathrm{TCP}$ & $\begin{array}{l}\text { UEs are clustered around transmitters, } \\
\text { seen as the parent points [126]-[128]. }\end{array}$ \\
\hline Uniformly random & Clustered & No & PPP & MCP, ТCP & $\begin{array}{l}\text { The analysis is focused on the clustering } \\
\text { aspect of UEs [160]-[162]. }\end{array}$ \\
\hline Clustered & Clustered & Yes & $\mathrm{TCP}$ & TCP & $\begin{array}{l}\text { UEs and BSs are clustered around the } \\
\text { same hotspots [129]. }\end{array}$ \\
\hline Uniformly random & Repulsive & Yes & PPP & PHP & $\begin{array}{l}\text { A typical application is when some UEs } \\
\text { are allowed to transmit only if they are } \\
\text { outside exclusion regions around specific } \\
\text { UEs or BSs [105], [107]. }\end{array}$ \\
\hline Repulsive & Uniformly random & No & $\begin{array}{l}\text { Regular lattice, } \\
\text { Soft- and hard-core } \\
\text { PPs. }\end{array}$ & PPP & $\begin{array}{l}\text { Typical configuration in rural areas where } \\
\text { repulsion is required between BSs without } \\
\text { necessarily coupling with UE locations } \\
\text { [91], [98], [113], [123], [163]. }\end{array}$ \\
\hline
\end{tabular}

average separation distance between nodes is greater enough to ignore the effect of singularity at the model (when $r=0$ ). However, this effect cannot be ignored in environments with higher path loss exponent [167] or networks with very high infrastructure density [36], [168], [169], where the single slope UPM is deemed as inaccurate. In fact, the SINR-invariance property obtained under the single slope UPM [18], such that the overall SINR is independent from infrastructure density in the interference-limited regime, has reopened the discussion on the reliability of UPM since it is not conceivable that splitting cells indefinitely through the addition of new BSs, will maintain the same SINR distribution. A key aspect to overcome this limitation is to revisit the single slope UPM. The authors of [36], [168] introduced the multi-slope UPM (model \#2.1 in Table V) enabling to ascertain that the SINR-invariance property is no longer valid when the near-field path loss exponent is surprisingly under the dimension of the network, which turns out to near-universal outage as network density increases. A similar effect is assessed when considering the bounded path loss model (BPM) in [170], the single slope UPM accounting for BSs antenna elevation in [73], and the stretched exponential path loss model in [171].

Furthermore, based on extensive field measurements, it has been reported in [172] and the references therein that mmWave signals are very sensitive to blockages as compared to sub-6 GHz. Hence, considering LOS and NLOS paths in such environment is of great importance. That is, the authors of [71], [164] considered a revisited path loss model that incorporates LOS and NLOS transmissions, as

$$
\ell(r)= \begin{cases}\ell_{\text {los }}(r) & \text { with probability } p_{\text {los }} \\ \ell_{\text {nlos }}(r) & \text { with probability } p_{\text {nlos }}=1-p_{\text {los }}\end{cases}
$$

In [86], [165], the authors included an outage state in addition to LOS and NLOS states to accurately capture the sensitivity of mmWave communications to blockages. Generally, the model in (35) is a building block for other sophisticated models depending on the approximation of the LOS probability $p_{\text {los }}$ and the preferences for $\ell_{\text {los }}$ and $\ell_{\text {nlos }}$. For instance, the authors of [72] introduced a composite model of (35) and the multi-slope UPM wherein the LOS probability is mapped with representative models adopted by the 3rd generation partnership project (3GPP). The LOS probability in [173] is approximated with a modified sigmoid function to characterize the air-to-ground (AtG) channel in UAV-aided communication networks. A comprehensive survey of channel modeling for UAV communications can be found in [174].

\section{Various Cell Association Strategies}

In microwave ( $\mu$ Wave, i.e., sub-6GHz) multi-tier wireless networks, various layers of BSs are deployed, where BSs of the $i$ th tier $\Psi_{i}(i=1, \ldots, K)$ transmit data with a given transmit power $p_{i}$. In such a context, shadowing is a slowly varying effect and the typical UE located at $y$ commonly selects the 
TABLE V

KEY PATH LOSS MODELS USED IN STOCHASTIC GEOMETRY ABSTRACTION

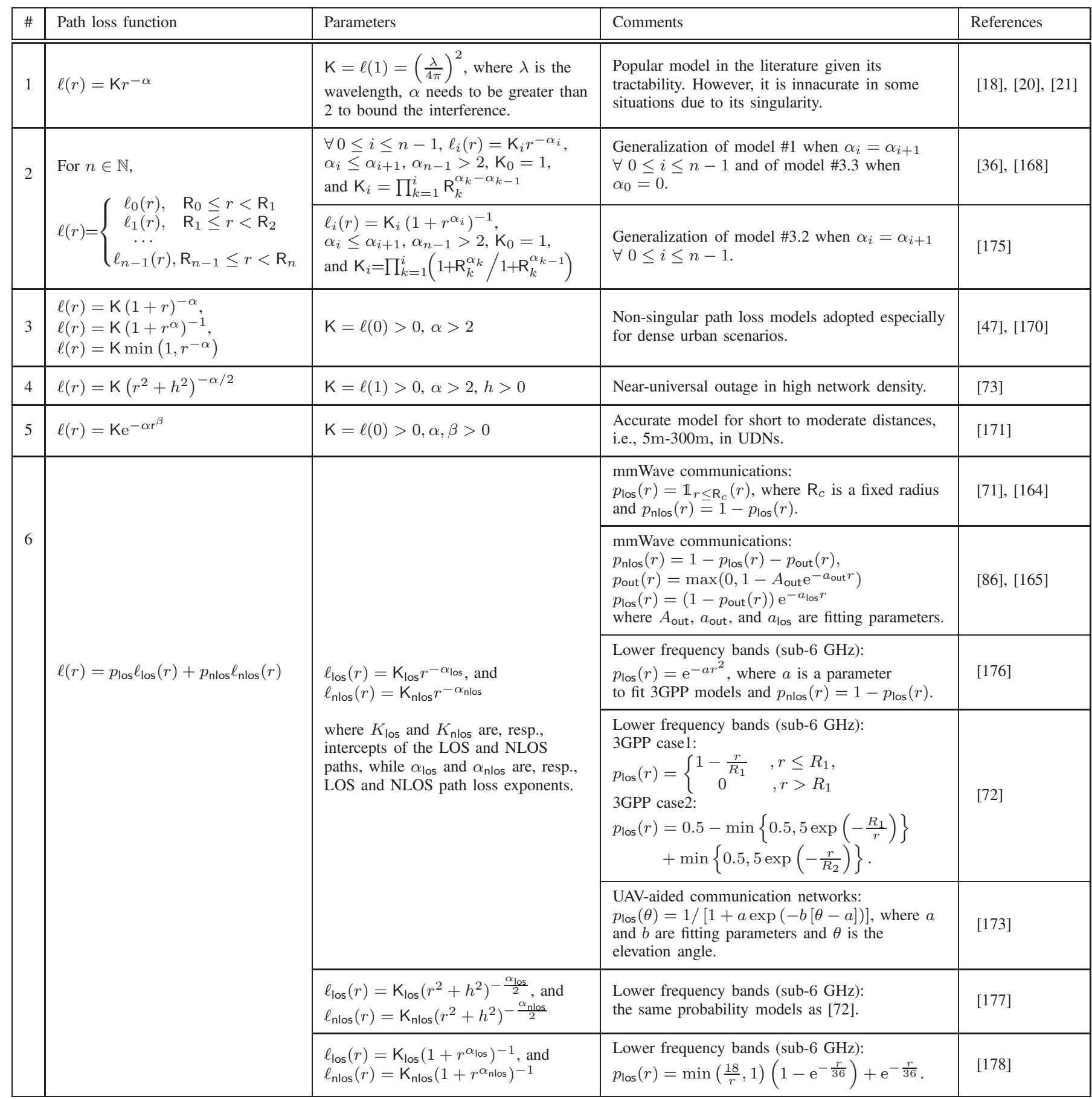

serving BS $x_{0}$ based on the strongest average received power strategy (without fading) [26], [61], as

$$
x_{0}=\arg \max _{x \in \Psi_{i}, \forall i=1, \ldots, K} p_{i} \ell(\|x-y\|),
$$

where $\ell($.$) is the path loss function (see Table V).$

Expression (36) induces then an exclusion region of radius $\delta_{0}$ around $y$ wherein no interfering $\mathrm{BS}$ to $x_{0}$ exists. That is, $\delta_{0}$ is expressed as

$$
\delta_{0}=\min _{x_{0} \in \Psi_{i}, \forall j=1, \ldots, K}\left\{\ell^{-1}\left(\frac{p_{i}}{p_{j}} \ell\left(\left\|x_{0}-y\right\|\right)\right)\right\} .
$$

However, the association criterion in (36) may sometimes lead to heavily loaded BSs especially those transmitting with the highest power, i.e., macro BSs, which reduces the average achievable rate and the efficiency of deploying small cells. An alternative way is then to associate users with BSs providing the highest data rate [44], [179], which can be captured via a measure of BSs load. Accordingly, the authors of [21] endowed BSs of each tier $i$ by some adjustable bias $B_{i}$, where the typical UE $y$ selects the serving BS as that providing the maximum average power weighted by its bias, namely the 
biased cell association. (36) becomes

$$
x_{0}=\arg \max _{x \in \Psi_{i}, \forall i=1, \ldots, K} p_{i} B_{i} \ell(\|x-y\|) .
$$

Bias $B_{i}$ can then improve the capacity of HetNets by offloading users from overloaded cells to lighter ones, namely, load balancing, which is similar to cell breathing through cell range expansion (CRE) [180], [181].

In the single-tier case where BSs send data with the same transmit power, using the association strategy in (36) turns into associating users with their spatially closest BS, which is equivalently named the nearest-neighbor cell association [18], [61], [73], [140], or extended to the nth nearest serving BS policy as [16], [19], [46], [59]-[61], [63]. Furthermore, considering system models incorporating various propagation groups, with various path loss exponents (model \#6 in Table $\mathrm{V}$ ), the association policy in (36) is equivalently referred to as the smallest path loss cell association [71], [72], [165].

Last but not least, in environments where shadowing is expected to be less slowly-varying, e.g., sensitive transmissions to blockages, interferers may be closer to the typical UE than the serving BS and then no exclusion region in (37) is considered. That is, we need to consider the shadowing effect $Z_{x}$, which renders that the typical UE connects to the strongest BS instantaneously, namely the maximum instantaneous powerbased cell selection, or equivalently the max-SINR association policy [20], [26], [58], [141], such that

$$
x_{0}=\arg \max _{x \in \Psi_{i}, \forall i=1, \ldots, K} p_{i} Z_{x} \ell(\|x-y\|) .
$$

It is worth mentioning that SG-based modeling and analysis of wireless networks under the previous association policy has taken two directions: i) the first by resorting to the Campbell theorem (15) as in [20], [26], [58], [88], [89], [141], ii) the second by absorbing the shadowing effect into the intensity of a new PPP as in [55], [58], [86], [88], [164], [165], and hence (39) will be consistent with (36).

\section{Transmitter-Receiver Direction of Analysis}

In downlink wireless networks, the analysis is generally focused on the received SINR at the level of the typical UE served by one or more BSs [18], [20], [21], [58]. However, with the growing interest in symmetric traffic applications, e.g., cloud-storage, the uplink performance analysis is becoming increasingly crucial [22], [152]-[159]. Typically, analytical evaluation of uplink wireless networks is generally involved as compared to the downlink, due to the following fundamental changes in the system model: First, the use of locationdependent power control, where each UE smoothly adjusts its transmit power to partially/totally invert the effect of path loss [22], [152]-[154] and/or log-normal shadowing [155], which mitigates the uplink interference and reduces the UE battery consumption. Second, the dependency in the location of concurrent uplink UEs. These uplink modeling aspects render the approximation of the users PP less accurate.

Interestingly, the authors of [156]-[158], considered the paradigm of decoupled uplink-downlink access (DUDA), where different association policies are considered for uplink and downlink inducing that the typical UE will not necessarily be prompted to access the same BS for both directions. The DUDA capability is particularly relevant in the scenario of emerging HetNets [20], [21], [26], [44], [58], wherein users quality of experience (QoE) is affected by non-uniformity in transmit powers and traffic loads in both downlink and uplink. In such a context, DUDA enables to reduce the transmit power of edge users, which obviously helps to reduce the average uplink interference by about $2-3 \mathrm{~dB}$ (see [158] and references therein).

Last but not least, the works in [53], [182] considered the IBFD capability enabling to transmit and receive data simultaneously over the same frequency band, which offers the opportunity to double the spectral efficiency at the expense of extra self interference. More discussions about the use of SG for modeling and analysis of IBFD approach as a potential enabler for 5G/B5G networks will be presented in Section V.

\section{E. Modeling Nodes' Mobility}

The mobility of transmitters and receivers is a crucial component in the design and performance evaluation of modern wireless networks since it can impinge on traffic load per cell, signaling protocols, handoff algorithms, and location update mechanisms. However, modeling human mobility is generally challenging given its very complex temporal and spatial correlation [183]. A comprehensive survey of user mobility models can be found in [184].

SG as a powerful mathematical tool has been explored in user mobility-aware performance analysis of wireless networks. Based on the formalization of the handoff rate, there are typically two directions of analysis adopted in the literature: i) the trajectory-based handoff, in which the handoff event occurs as well as the mobile UE crosses a cell border, and then the handoff rate is defined as the average number of crossing cell boundaries of different cells by a moving UE. Hence, the accuracy of such a concept is biased by the efficiency of quantifying the statistical distribution of cells boundary, which is generally consistent with the Buffon's needle problem (see Section I). The work in [3] is the first to consider this approach in an SG-based framework, where BSs are modeled as a 2D PPP, the road system as a PLP, and the location of users as a 1D PPP on the road layout (see Fig. 6). In [185], the authors considered a tractable model for user's mobility, namely the random waypoint $(R W P)$, where a detailed description can be found in [184]. Next, the authors derived the distribution of UE location during one movement period, the handover rate, and the average time of being served by a given BS, also known as the sojourn time. Other representative works using the same approach in the context of HetNets can be found in [186]-[189]. ii) The second direction is based on the association-based handoff where the handoff event occurs as long as another BS verifies the association criterion better than the current serving BS. In this way, the handoff rate is defined as the probability of inducing a handoff for a UE served by a given BS and moving a random distance in a unit time [190]-[192]. A comprehensive tutorial of mobilityaware performance analysis considering spatially random and deterministic grid-based topologies is given in [193]. 
In future wireless networks, BSs can also be enabled to move seamlessly in order to provide enhanced service to UEs. In this way, two important applications are identified in the literature. First, given their agility and flexible deployment, UAVs can be designed as airborne BSs to support coverage and capacity for various UEs. In particular, it has been demonstrated by aerodynamic theory that there is an operational UAV speed that provides the optimal energy consumption [194]. Thus, a network of moving aerial BSs can outperform a static hovering one in terms of energy consumption. Also, mobile aerial BSs have the potential to reduce the average time that the typical UE experiences blockages, namely the average fade duration. However, uniformly distributed static aerial BSs can provide fairly uniform coverage over the entire service area. In this way, the authors of [194] considered tools from SG to develop a family of trajectory processes (i.e., spiral and oval) for mobile aerial BSs to remain BPP-distributed, thus ensuring uniform coverage over the service area as in the static case [195]. In [196], the ergodic rate received by the typical UE is evaluated under a setup where four canonical mobility models are adopted for interfering aerial BSs and two service models for the serving aerial BS. Using the displacement theorem to capture the new PP resulting after running each service and mobility model, the results showed that the tractable straightline mobility model inspired from the 3GPP simulation models can serve as a lower bound for system-level performance evaluation with more sophisticated mobility models.

Second, given the growing number of users using wireless connectivity when moving via public transportation vehicles (i.e. buses, tramways, or high-speed trains), a dedicated moving relay node (RN) is envisioned to ensure seamless connectivity of UEs in public transport. In particular, mobile RNs can create their own cell inside a vehicle, thus eliminating a high vehicular penetration loss (which can reach $25 \mathrm{~dB}$ at $2.4 \mathrm{GHz}$ ) for onboard UE service [197]. This improves the power consumption of such UEs and reduces the handoff signalling between them (i.e, grouped handoff). In addition, multiple backhaul antennas can be installed on the outer side of the mobile RN, allowing to establish a cooperative system with multiple static backhaul-enabled BSs, which improves propagation conditions and backhaul reliability for moving RNs. Using SG, the authors of [198] conceived a system model in which outdoor antennas of moving RNs can assist the coverage of static UEs located outside vehicles. Based on the correlation between the received power at the typical UE when the power is transmitted by the nearest BS or the nearest moving RN, a bias-based CoMP association criterion is considered to improve the coverage of UEs served by moving RNs. Considering the effect of blockages and handoffs is, however, an important extension of this analysis.

From previous discussions, it is clear that the statistical characteristics of the wireless cell boundaries are crucial in the design of handover schemes. Typically, the wireless cell boundaries resulting from the Delaunay triangulation method or from the received power at edge UEs, divide the service area into several irregular polygons representing different wireless cellular coverage areas. Hence, for simplicity, the received power is assumed to be identical at all points with equal distances to the BS, which leads to a smooth wireless cellular boundary at small scales. This assumption does not take into account the anisotropy of path loss fading in real urban wireless propagation environments due to the irregular distribution of blockages and the effect of interference and load in different directions from the BS. Importantly, field measurements have shown that wireless coverage boundaries are not smooth at small scales and exhibit statistical fractal characteristics at angular scales [199]. An alpha-stable distribution with the heavy tail feature is thus used to fit the PDF of the wireless cell coverage boundary. Based on SG, the authors of [200] proposed a multi-directional path loss model for fractal small cell networks, where the path loss exponent is modeled by i.i.d. random variables depending on the direction of signal propagation. Also, the association-based handoff abstraction is considered to derive the handoff rate under anisotropic path loss, which confirmed the importance of accurate wireless cell boundaries in the design of handoff parameters.

\section{F. Spatio-temporal Traffic Modeling}

Emerging new data-intensive applications, such as multiparty video conferencing or multiplayer online games, along with latency-critical applications such as smart manufacturing or autonomous driving, suggest that the integration of spatiotemporal traffic dynamics in the analysis of 5G/B5G wireless networks will play an increasingly crucial role in their design and deployment. Previous efforts have typically considered one aspect of traffic: i) abstraction based on queuing theory, which primarily evaluates scheduling algorithms and ignores the interaction of traffic with SINR statistics and hence with network geometry; ii) SG-based analysis, which usually does not consider the temporal arrival process of packets and focuses on reliability or throughput in fully buffered networks, i.e. each link always has a packet to send. Interestingly, traffic has recently been abstracted by spatio-temporal modeling that combines tools from SG and queuing theories. In particular, the spatial domain of traffic is captured by modeling nodes via an appropriate PP, while the temporal variation of traffic is captured by the temporal arrival of packets grasped tractably by independent Bernoulli processes [201], [202].

A review of the literature shows that spatio-temporal traffic modeling has been particularly exploited to evaluate two important metrics of interest. First, delay that refers to the end-to-end duration from packet initiation at the transmitter to successful decoding at the receiver [203]. This includes the delay in generating a packet, the delay in queuing it, and then the time it takes for the packet to be successfully transmitted within the wireless access network and backhaul links (including the delay incurred in the retransmission mechanism). Delay analysis through spatio-temporal modeling is generally challenging due to the following major issues: i) The delay is dependent on the system throughput determined by the SINR, which in turn relies on the network geometry and the complex channel fluctuations in large-scale networks. ii) The delay is a long-term measure in which the topology of nodes remains static but random for a fairly long time, which induces a coupling of interference across various time slots. iii) As 
part of the MAC, a scheduling policy is performed on many queuing nodes in a distributed manner. So, if a queue is idle, the related transmitter does not interfere with the other links; as a result, the service throughput of those links increases allowing their queues to drain faster. Such coupling between each queue and the state of all the other queues renders delay analysis less tractable. To make the analysis feasible, most of the literature focuses on the queuing delay based on the scheduling scheme, and the transmission delay related to the number of transmission trials required until a packet is successfully decoded, while the delay in backhaul links is generally omitted. A comprehensive example of backhaul link delay analysis can be found in [204]. Next, the mean delay is evaluated conditioning on a given realization of the PP, which resorts to a formulation based on the meta-distribution metric that can be derived by applying the Gil-Pelaez theorem or the $k$-moment inversion. More discussions about the metadistribution and the Gil-Pelaez technique are brought in the sequel.

Second, the emerging metric of age of information (AoI) that measures freshness (timely updating) of the sensed data measurements of the IoT devices at the destination nodes. It is typically defined as the time expired since the previous successfully received update packet at the destination was generated at the source [205]. In this way, the requirement for timely updating actually reflects a small average age of status update, i.e., minimizing time-average AoI, which can help in the efficient design of freshness-aware IoT systems. It is worth mentioning that minimizing AoI does not really correspond to maximizing the system throughput, nor guaranteeing a minimum delay in receiving IoT measurements. Intuitively, throughput can be maximized by making sensors send updates as quickly as possible, which can result in higher AoI because the status messages will be pending/backlogged in the communication system. In such a context, reducing the system throughput may improve the AoI. It is worth mentioning that the optimal loading usually requires a perfect balance between overloading the queue and keeping it idle.

Since characterizing the distribution of AoI based on spatiotemporal traffic modeling is known to be notoriously difficult, efforts are mainly devoted to studying some of its easyconstructed variants. For instance, i) work with a lower bound on the average AoI obtained by ignoring the processing time at the source, which mean that a new update packet is instantaneously generated by the source node [206], or ii) consider the peak AoI that quantifies the maximum value of the AoI immediately before an update packet is delivered to the destination node, thus yielding insights into the pessimistic values of the AoI [207], [208]. A comprehensive tutorial about SG analysis of spatio-temporal performance in wireless networks can be found in [209].

\section{G. Key Performance Metrics}

Since Shannon's work [210], the received SINR has been considered as the first-order predictor of link reliability and users QoE, where almost all performance metrics conceived to date are closely related to the SINR. For instance, the bit error

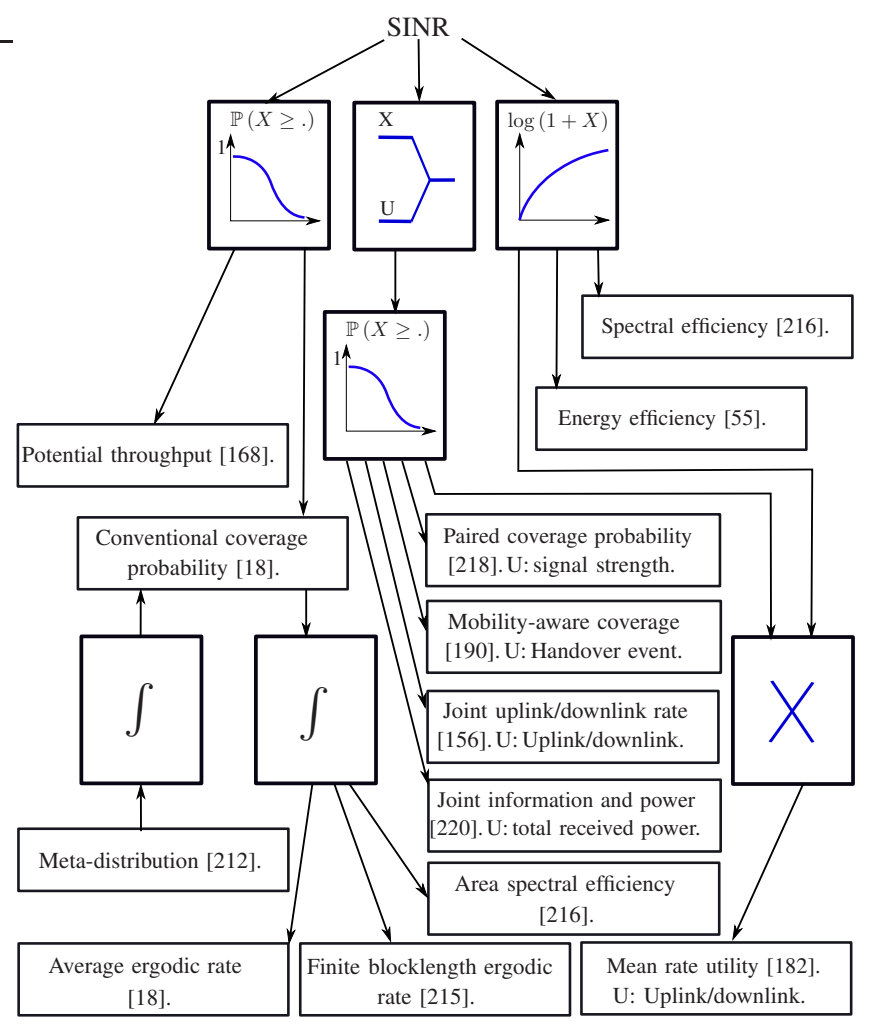

Fig. 10. Main performance metrics used in the SG abstraction.

rate depends on $\mathcal{Q}(a \sqrt{\operatorname{SINR}})$ [5, Claim 24.3.4] and the data rate follows $\log (1+\operatorname{SINR})$ [5, Definition 16.2.5], where $\mathcal{Q}($. is the $\mathcal{Q}$-function and $a$ is a constant depending on modulation and detection.

A review of the largely fragmented literature of SG-based studies for modeling and analysis of wireless networks reveals that almost all the adopted performance metrics are typically based on six key operations of SINR, as illustrated in Fig. 10. In the following, we will consider the definition of key representative performance metrics.

1) Spectral efficiency: The spectral efficiency $S_{e}$ is conceived as the maximum information rate that can be transmitted over a given bandwidth B. In the simplest case of AWGN and optimal theoretical link performance, the Shannon-Hartley theorem defines $S_{e}$ in units of [nats/s] as

$$
\mathrm{S}_{\mathrm{e}} \triangleq \mathrm{B} \log (1+\mathrm{SINR}) \text {. }
$$

2) Energy efficiency (EE): The EE $\mathcal{E}$ evaluates the number of bits that can be successfully transmitted with unit energy. It is generally expressed under the form [55], [142], [211]

$$
\mathcal{E}=\frac{\mathrm{S}_{\mathrm{e}}}{\varpi P_{\mathrm{tx}}+\alpha},
$$

where $P_{\mathrm{tx}}$ is the BS transmit power and $\varpi, \alpha$ are some positive constants depending on the power consumption model.

3) Mean rate utility: It is particularly defined in the context of a generic IBFD link [182], as

$$
\mathcal{R}_{\mathrm{u}}\left(\mathrm{T}_{\mathrm{DL}}, \mathrm{T}_{\mathrm{UL}}\right)=\mathbb{P}\left(\mathrm{S}_{\mathrm{e}}^{\mathrm{DL}} \geq \mathrm{T}_{\mathrm{DL}}\right) \mathrm{S}_{\mathrm{e}}^{\mathrm{DL}}+\mathbb{P}\left(\mathrm{S}_{\mathrm{e}}^{\mathrm{UL}} \geq \mathrm{T}_{\mathrm{UL}}\right) \mathrm{S}_{\mathrm{e}}^{\mathrm{UL}},
$$

where $T_{D L}$ and $T_{U L}$ are, respectively, the required spectral efficiency thresholds in the downlink and the uplink. 
4) Conventional coverage probability: The coverage probability $P_{c}$, as opposed to outage probability $P_{o}$, is defined as the probability that the typical user can reach a target SINR, $\mathrm{T}$ [18]. It is expressed as

$$
\mathrm{P}_{c}(\mathrm{~T})=1-\mathrm{P}_{\mathrm{o}}(\mathrm{T})=\mathbb{P}(\operatorname{SINR} \geq \mathrm{T}),
$$

which also can be interpreted as the success probability of the typical transmission/link averaged over all spatial links [7], [47], [109]. Formally, we first condition on the BS process and the typical UE located at the origin $x_{0}$ of the PP $\Psi$, and next average over all the spatial links, as

$$
\mathrm{P}_{c}(\mathrm{~T})=\mathbb{E}\left(\mathbb{P}^{o}(\operatorname{SINR} \geq \mathrm{T} \mid \Psi)\right) .
$$

5) Meta-distribution: Expression (44) can be rephrased as the reduced Palm expectation over the PP realization, which does not provide insights about how concentrated are the well covered areas or what are the link success probabilities. The meta-distribution concept is introduced in [212] to obtain finegrained information about the performance, as

$$
\bar{F}(\mathrm{~T}, u)=\mathbb{P}\left(\mathbb{P}^{o}(\operatorname{SINR} \geq \mathrm{T} \mid \Psi)>u\right), u \in[0,1] .
$$

The coverage probability in (44) becomes then

$$
\mathrm{P}_{\mathrm{c}}(\mathrm{T})=\int_{0}^{1} \bar{F}(\mathrm{~T}, u) \mathrm{d} u=\lim _{u \rightarrow 1} \underbrace{\int_{0}^{u} \bar{F}(\mathrm{~T}, x) \mathrm{d} x}_{\mathrm{P}_{\mathrm{c}}(\mathrm{T}, u)} .
$$

Hence, the meta distribution concept can be seen as based on some philosophy of derivation from the distribution of SINR, i.e., $\bar{F}(\mathrm{~T}, u)=\partial \mathrm{P}_{\mathrm{c}}(\mathrm{T}, u) / \partial u$. The derivation construct actually aims to capture variability at particular points on a curve or any other geometric shape. Similarly, meta-distribution aims to evaluate fine-grained information on the distribution of the SINR.

6) Average ergodic rate: Another quantity of interest is the average ergodic rate $\tau$, also known as the Shannon throughput, accounting for the mean data rate achievable over a cell. It is obtained in units of [nats/s/Hz] as [18], [20], [21]

$$
\tau \triangleq \mathbb{E}(\log (1+\operatorname{SINR}))
$$

The average ergodic rate in (47) may actually require the preliminary calculation of $P_{c}$ [17], [18], [70], since

$$
\begin{aligned}
\tau & =\int_{t>0} \mathbb{P}(\log (1+\operatorname{SINR})>t) \mathrm{d} t \\
& =\int_{t>0} \mathbb{P}\left(\operatorname{SINR}>\mathrm{e}^{t}-1\right) \mathrm{d} t \\
& =\int_{x>0} \frac{\mathrm{P}_{\mathrm{c}}(x)}{x+1} \mathrm{~d} x .
\end{aligned}
$$

7) Finite blocklength ergodic rate: From an informationtheoretic angle, (47) is a reasonable performance metric for modern wireless networks supporting enhanced mobile broadband (eMBB) services where codewords length is sufficiently large to maximize throughput and induce very small packet error probability. However, in the context of ultra-reliable and low latency communications (URLLC), the throughput is not a key requirement of the system, and the tradeoff between low latency and ultra-high reliability requires generally the use of short packets [213] [214]. In such a context, the ergodic rate of communication is approximated as [215, Equation 296]

$$
\tau^{*}(n, \epsilon) \approx \mathbb{E}\left(\mathrm{C}-\sqrt{\frac{V}{n}} \mathcal{Q}^{-1}(\epsilon)+\frac{1}{2 n} \log (n)\right),
$$

where $n$ is the blocklength, $\epsilon$ is the error probability, $\mathrm{C}=$ $\log (1+$ SINR $)$ is the capacity of an AWGN channel, and $V$ is the channel dispersion approximated as a function of SINR in [215, Equation 293].

8) Area spectral efficiency: The concept of area spectral efficiency (ASE) has been introduced for the first time in [216, Equation (65)] to measure, for a partially loaded system, the maximum average data rate per unit area per unit bandwidth supported by a cell. Formally,

$$
\mathrm{ASE}=\frac{1}{|\mathcal{A}|} \sum_{k=1}^{N_{\mathrm{s}}} \mathbb{E}\left(\log \left(1+\operatorname{SINR}_{k}\right)\right),
$$

where $|\mathcal{A}|$ is the area of interest, $N_{\mathrm{s}}$ is the total number of active UEs inside $|\mathcal{A}|$, and $\mathbb{E}\left[\ln \left(1+\mathrm{SINR}_{k}\right)\right]$ is the ergodic rate of the $k$ th $\mathrm{UE}$.

Under the SG abstraction, ASE in (50) can be simplified as

$$
\mathrm{ASE}=\lambda \mathbb{E}(\log (1+\operatorname{SINR})),
$$

where the expectation averages over different network and fading realizations, and $\lambda$ is the density of active BSs [217].

In realistic scenarios, a minimum SINR constraint $\gamma_{0}$ is required for the system operational regime [72], which induces a constrained variant of the area spectral efficiency as

$$
\mathrm{ASE}_{\mathrm{c}}=\lambda \mathbb{E}\left(\log (1+\operatorname{SINR}) \mathbb{1}\left(\operatorname{SINR} \geq \gamma_{0}\right)\right) .
$$

9) Potential throughput: The potential throughput considered in [86], [168], [171], is another variant of the area spectral efficiency. It is defined in units of $\left[\mathrm{bps} / \mathrm{Hz} / \mathrm{m}^{2}\right]$ as

$$
\mathrm{P}_{\mathrm{th}}(\mathrm{T})=\lambda \log (1+\mathrm{T}) \mathbb{P}\{\mathrm{SINR} \geq \mathrm{T}\} .
$$

Interestingly, it has been demonstrated in [37] that (51), (52), and (53), are ordered as follows

$$
\mathrm{P}_{\mathrm{th}} \leq \mathrm{ASE}_{\mathrm{c}} \leq \mathrm{ASE}
$$

10) Paired coverage probability: A new definition of coverage probability is considered in [218], [219], such that the typical UE is in coverage if, i) it receives a sufficiently good signal strength, i.e., the short-term average signal-tonoise ratio $\overline{\mathrm{SNR}}$ is greater than a certain threshold $\mathrm{T}_{\mathrm{s}}$, and ii) it receives a good signal quality, i.e., the SIR is greater than another threshold $\mathrm{T}_{\mathrm{q}}$. Formally,

$$
\mathrm{P}_{\mathrm{c}}\left(\mathrm{T}_{\mathrm{s}}, \mathrm{T}_{\mathrm{q}}\right)=\mathbb{P}\left(\overline{\mathrm{SNR}} \geq \mathrm{T}_{\mathrm{s}}, \mathrm{SIR} \geq \mathrm{T}_{\mathrm{q}}\right)
$$

(54) is shown to capture more system-level parameters than (43), and enables deriving tractable closed-form expressions. 
11) Mobility-aware coverage probability: In [190], [191], the authors introduced a mobility-aware coverage probability, where handoffs may cost service delays or drops. (43) is updated as

$$
\mathrm{P}_{\mathrm{c}}(\mathrm{T})=\mathbb{P}(\mathrm{SINR} \geq \mathrm{T}, \overline{\mathrm{H}})+(1-\beta) \mathbb{P}(\mathrm{SINR} \geq \mathrm{T}, \mathrm{H}),
$$

where $\mathrm{H}$ is the handoff event, and $\beta \in[0,1]$ reflects system sensitivity to QoS impairment when handoff occurs.

12) Joint uplink and downlink rate coverage: It is defined as the fraction of users with sufficient spectral efficiency (or SINR) in the uplink and downlink simultaneously [156]. It is expressed as

$$
\mathcal{R}\left(\mathrm{T}_{\mathrm{DL}}, \mathrm{T}_{\mathrm{UL}}\right)=\mathbb{P}\left(\mathrm{S}_{\mathrm{e}}^{\mathrm{DL}} \geq \mathrm{T}_{\mathrm{DL}}, \mathrm{S}_{\mathrm{e}}^{\mathrm{UL}} \geq \mathrm{T}_{\mathrm{UL}}\right) .
$$

13) Joint information and power coverage: The joint information and power coverage $\mathcal{P}$, is introduced in [220], [221] to evaluate the performance of simultaneous wireless information and power transfer (SWIPT). It is is expressed as

$$
\mathcal{P}\left(\mathrm{T}_{\mathrm{i}}, \mathrm{T}_{\mathrm{e}}\right)=\mathbb{P}\left(\mathrm{S}_{\mathrm{e}} \geq \mathrm{T}_{\mathrm{i}}, \mathrm{E} \geq \mathrm{T}_{\mathrm{e}}\right),
$$

where $E$ is the total received power at the energy harvester.

\section{H. Analytical Techniques}

As discussed before, using non-PPPs helps to accurately capture the system behavior but reduces tractability and mathematical flexibility, which requires resorting to efficient numerical integration (e.g., quasi Monte-Carlo integration method [110]), or even intractable approximations with limited impact on design insights [110, Equation (25)], [113, Equation (42)], [123, Equation (22)]. Subsequently, we will focus on key approaches considered under the PPP seen as the reference PP. We will also consider its finite version, the BPP. To the best of authors knowledge, eleven techniques are reported in the literature, offering varying degrees of tractability, accuracy, and mathematical flexibility.

To illustrate the key generative sequence steps of each technique, we consider the general common definition of the received SINR at the level of the typical user located in $y \in \mathbb{R}^{d}$ from a serving $\mathrm{BS} x_{0}$, as

$$
\operatorname{SINR}\left(x_{0} ; y\right)=\frac{h / \mathrm{L}\left(R_{0}\right)}{I+W},
$$

where $\ell()=.1 / \mathrm{L}($.$) is the path loss function (see Table \mathrm{V}$ ). I is the power of the other-cell interference normalized by the BS transmit power $P_{\mathrm{tx}}$, and can be expressed as

$$
I=\sum_{k \in \Psi \backslash\left\{x_{0}\right\}} g_{k} / \mathrm{L}\left(R_{k}\right),
$$

where $\left(x_{k}\right)$ are BSs location modeled by a HPPP $\Psi$ of density $\lambda, x_{0}$ is the serving BS under a given association strategy, $R_{k}=\left\|x_{k}-y\right\|$ is the Euclidean distance between the BS $x_{k}$ and the typical user $y, h$ and $\left\{g_{k}\right\}_{k}$ are, respectively, fading coefficients of the serving BS and interferers, and $W$ is the noise power normalized by $P_{\mathrm{tx}}$.
1) The baseline two-step approach: This is the most popular technique used in the literature to derive coverage probability in (43). In fact, assuming Rayleigh ${ }^{12}$ fading for the desired link, the approach consists on first computing the coverage probability by conditioning on $R_{0}$ and next averaging w.r.t it. Accordingly, for $h \sim \exp (1)$, the coverage probability in (43) is simplified as

$$
\begin{aligned}
\mathrm{P}_{\mathrm{c}}(\mathrm{T}) & =\mathbb{P}\left(h \geq \mathrm{TL}\left(R_{0}\right)(I+W)\right) \\
& =\mathbb{E}_{R_{0}}\left(\mathbb{P}\left(h \geq \mathrm{TL}\left(R_{0}\right)(I+W) \mid R_{0}\right)\right) \\
& \stackrel{(\mathrm{a})}{=} \mathbb{E}_{R_{0}}\left(\mathcal{L}_{W}\left(\operatorname{TL}\left(R_{0}\right)\right) \mathcal{L}_{I}\left(\operatorname{TL}\left(R_{0}\right)\right)\right),
\end{aligned}
$$

where (a) follows from the Laplace transform definition and the independence between $W$ and $I$.

The expectation in (62) is generally expressed under the form $\mathbb{E}_{R_{0}}\left(\varphi\left(R_{0}\right)\right)=\int_{0}^{\infty} \varphi(x) f_{R_{0}}(x) \mathrm{d} x$, where the function $f_{R_{0}}($.$) reflects a unified framework of the BS association$ scheme [73]. Typically, when considering the nearest-neighbor cell association [18], [21], [61], [73], [140], $f_{R_{0}}($.$) is the PDF$ of the random variable $R_{0}$, as $f_{R_{0}}(\xi)=2 \pi \xi \mathrm{e}^{-\pi \lambda \xi^{2}}$. However, if the max-SINR association is considered [20], [26], [58], [141], $f_{R_{0}}(\xi)=2 \pi \lambda \xi$. Besides, the Laplace transform of the interference can be expressed via the PGFL theorem (16) as [18], [73]

$$
\begin{aligned}
& \mathcal{L}_{I}\left(\operatorname{TL}\left(R_{0}\right)\right)= \\
& \exp \left(-\pi \lambda \mathbb{E}_{g}\left(\int_{\vartheta\left(R_{0}\right)}^{\infty}\left(1-\exp \left(-g \frac{\mathrm{TL}\left(R_{0}\right)}{\mathrm{L}(u)}\right)\right) u \mathrm{~d} u\right)\right),
\end{aligned}
$$

where $\vartheta($.$) captures the exclusion region of interferers. Typ-$ ically, $\vartheta(x)=x$ in the nearest-neighbor cell association, where interferers cannot be closer to the typical UE than the serving BS. In the max-SINR association, no exclusion region is considered for interferers and $\vartheta(x)=0$.

Interestingly, (63) can be further simplified using variable changes as in [70, Equation (34)]. If interference signals are also experiencing Rayleigh fading, (63) will be simplified as

$$
\mathcal{L}_{I}\left(\mathrm{TL}\left(R_{0}\right)\right)=\exp \left(-\pi \lambda \int_{\vartheta\left(R_{0}^{2}\right)}^{\infty} \frac{\mathrm{d} x}{1+\frac{\mathrm{L}(\sqrt{x})}{\mathrm{TL}\left(R_{0}\right)}}\right) .
$$

Despite the Rayleigh assumption on the intended signal and interferers, coverage probability in (62) is generally expressed under an improper integral requiring a two-fold numerical integration [18, Theorem 1]. Some efforts are made in the literature to derive closed-form expressions or approximations of the coverage probability. For instance, tractable expressions are obtained in [18] by assuming the interference-limited regime or a path loss exponent equals to 4. In [87], a more generalized closed-form expression is proposed assuming an integer value of the path loss exponent. In [222], four approximation techniques are proposed based on the network

\footnotetext{
${ }^{12}$ The Rayleigh assumption is generally supported by i) its better tractability and mathematical flexibility incorporated into analysis [18], [20], [21], [58], and ii) its ability to give a pessimistic version of the SINR distribution as compared to more realistic fading models, e.g., Nakagami fading [147].
} 
operational regime. It has particularly shown that the optimal approximation is achieved by combining the four techniques in accordance with their convergence properties.

2) Coverage probability new abstraction: To develop a closed-form expression of the coverage probability in (62), it has been proposed in [218], [219] to bound the upper endpoint of the integral in (62) by introducing the concept of the paired coverage probability as in (54). That is, (54) is simplified as

$$
\begin{aligned}
\mathrm{P}_{\mathrm{c}}\left(\mathrm{T}_{\mathrm{s}}, \mathrm{T}_{\mathrm{q}}\right) & =\mathbb{P}\left(\frac{h \mathbb{1}\left\{\mathrm{L}\left(R_{0}\right) \leq 1 /\left(W \mathrm{~T}_{\mathrm{s}}\right)\right\}}{I \mathrm{~L}\left(R_{0}\right)} \geq \mathrm{T}_{\mathrm{q}}\right) \\
& =\int_{0}^{\mathrm{L}^{-1}\left(1 /\left(W \mathrm{~T}_{\mathrm{s}}\right)\right)} \mathcal{L}_{I}\left(\mathrm{~T}_{\mathrm{q}} \mathrm{L}(\xi)\right) f_{R_{0}}(\xi) \mathrm{d} \xi,
\end{aligned}
$$

which can be further simplified by considering common combinations of i) the standard path loss function of path loss exponent $\alpha$, ii) additive and constant thermal noise, and iii) the nearest-neighbor cell association, as

$$
\mathrm{P}_{\mathrm{c}}\left(\mathrm{T}_{\mathrm{s}}, \mathrm{T}_{\mathrm{q}}\right)=\frac{1-\exp \left(-\pi \lambda\left(\frac{W}{\mathrm{~T}_{\mathrm{s}}}\right)^{\frac{2}{\alpha}} F_{-\frac{2}{\alpha}}\left(\mathrm{T}_{\mathrm{q}}\right)\right)}{F_{-\frac{2}{\alpha}}\left(\mathrm{T}_{\mathrm{q}}\right)} .
$$

In [223], this technique has been considered to benchmark the performance of UDNs under three representative scheduling schemes in terms of fairness and implementation complexity. Assuming elevated BSs, closed-form expressions are obtained allowing to assess the network performance in a more tractable and meaningful fashion as compared to the conventional definition of coverage probability in (43).

3) The relative distance process (RDP) based approach: Based on the assumptions of: i) the standard path loss model with path loss exponent $\alpha$, ii) Rayleigh fading, iii) the nearest BS association policy, and iv) the interference-limited regime, a new way is considered to derive the coverage probability in (60) via the $\operatorname{RDP} \Psi^{\mathcal{R}}$ of the PPP $\Psi$, defined as [212]

$$
\Psi^{\mathcal{R}}=\left\{\frac{R_{0}}{R_{k}} \mid x_{k} \in \Psi \backslash\left\{x_{0}\right\}\right\} \subset[0,1],
$$

where its intensity measure is derived based on (11), as [145]

$$
\Lambda(\mathrm{d} r)=2 r^{-3} \mathrm{~d} r
$$

When $\Psi^{\mathcal{R}}$ is an IPPP, the PGFL can be expressed as

$$
\mathcal{G}_{\Psi^{\mathcal{R}}}^{\mathrm{IPPP}}\{f\}=\exp \left(-2 \int_{0}^{1} \frac{1-f(r)}{r^{3}} \mathrm{~d} r\right) .
$$

In the general case, $\Psi^{\mathcal{R}}$ is not an IPPP, and then (60) is derived based on $\mathcal{G}_{\Psi \mathcal{R}}^{\mathrm{IPPP}}$, as [145, Lemma 1]

$$
\begin{aligned}
\mathrm{P}_{\mathrm{c}}(\mathrm{T}) & =\mathbb{E}(\mathbb{P}(\operatorname{SIR}>\mathrm{T} \mid \Psi)) \\
& =\mathbb{E}\left(\prod_{y \in \Psi^{\mathcal{R}}} f(y)\right)=\frac{1}{1-\log \left(\mathcal{G}_{\Psi^{\mathcal{R}}}^{\mathrm{IPPP}}\{f\}\right)},
\end{aligned}
$$

where $f(y)=1 /\left(1+\mathrm{T} y^{\alpha}\right)$ due to the assumptions i)-iv) considered in this technique.
(70) is actually a special case of the $k$-th moment, since

$$
\begin{aligned}
M_{k}(\mathrm{~T}) & =\mathbb{E}\left((\mathbb{P}(\mathrm{SIR}>\mathrm{T} \mid \Psi))^{k}\right) \\
& \stackrel{(\mathrm{a})}{=} \int_{0}^{1} k u^{k-1} \bar{F}(\mathrm{~T}, u) \mathrm{d} u,
\end{aligned}
$$

where (a) comes from the meta-distribution expression in (45).

It is worth mentioning that $M_{1}(\mathrm{~T})$ is the coverage probability defined in (43). Moreover, using similar steps from (70) to (71), the expression of the $k$-th moment $M_{k}(\mathrm{~T})$ in (73) can generally be expressed in a closed-form expression. However, reshaping the meta-distribution from $M_{k}(\mathrm{~T})$, is an instance of the Hausdorff moment problem, which is to derive the inverse $k$-moment. For instance, two techniques have been considered in [212]. The first one is by inverting the $j \omega$-moment via the Gil-Pelaez theorem [247], which resulted in less tractable expressions of the meta-distribution requiring efficient numerical integration. The second technique is based on approximating the meta-distribution with a beta distribution, where the design parameters are fitted from the first and second moments. This approach has showed an impressive accuracy, but remains relevant only for the measurements considered in study and may diverge for more general setups. In [224], the authors proposed to reconstruct the PDF of the entire meta-distribution defined over the interval $[0,1]$, by means of shifted Jacobi polynomials via Fourier-Jacobi expansion. The obtained series expansion is infinite, where the coefficients are mapped with the moments of $\mathbb{P}(\operatorname{SIR}>\mathrm{T} \mid \Psi)$ via the binomial expansion. The approach is promising, but needs more investigation on the convergence conditions. In [225], the authors explored binomial mixtures properties to obtain an approximation of the meta-distribution as a function of a finite double sum of the moment sequence. That is, the accuracy of the approach increases with the length of the moment sequence, which requires however efficient numerical computation.

In [226], the meta-distribution analysis is generalized to PPs beyond the PPP, where the $k$-th moment of the conditional success probability for a stationary PP can be inferred from that of the PPP by using the same horizontal shift technique introduced in [143]-[145]. Further extensions of the metadistribution to other system setups may be found in [202], [227]-[233].

4) Finite networks assumption: Following a review of the existing state-of-the-art works on modeling and analysis of finite wireless networks, we can generally identify three streams of thoughts ordered in decreasing tractability and mathematical flexibility. The first, considers a typical setup where the reference receiver is located randomly in a compact $C \subset \mathbb{R}^{2}$, while BSs are uniformly randomly distributed in a disc [19] or a L-sided polygon [62] centered at the reference receiver. The second, extends the first model by considering an arbitrarily-located reference receiver in the disc [195], [234] or L-sided polygon of BSs [63]. The third setup considers an arbitrarily-located reference receiver in an arbitrarily-shaped area that contains finite BSs [64].

As an illustration of the generative analytical background, we consider a typical scenario, in which the reference receiver is arbitrarily located in a disk-shaped finite wireless network, 

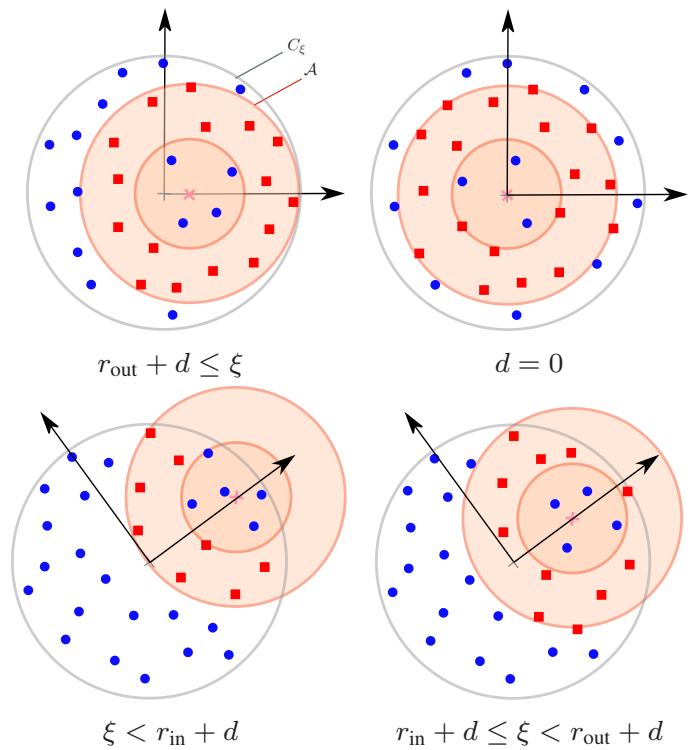

Fig. 11. The typical realizations of $C_{\xi}$ and $\mathcal{A}$ where the square red points are interferers.

wherein $N$ transmitting BSs are uniformly randomly distributed in a disc $C_{\xi}$ of radius $\xi$, i.e., $\Psi$ is a BPP. The reference receiver is located at a distance $0 \leq d \leq \xi$ from the origin of $C_{\xi}$ and interfering BSs are assumed to be exclusively located in an annular region $\mathcal{A}$ of inner radius $r_{\text {in }}$ and outer radius $r_{\text {out }}$ from the reference receiver, such as $0 \leq r_{\text {in }}<r_{\text {out }}<\xi$. Fig. 11 illustrates the typical realizations of $C_{\xi}$ and $\mathcal{A}$.

The density of the BPP is $\lambda=N / \pi \xi^{2}$, while the probability of having $k \leq N$ interferers inside $\mathcal{A}$ is

$\mathbb{P}(\Psi(\mathcal{A})=k)=\left(\begin{array}{c}N \\ k\end{array}\right) p^{k}(1-p)^{N-k}$, where $p=\frac{\left|\mathcal{A} \cap C_{\xi}\right|}{\pi \xi^{2}}$.

Given Rayleigh fading on the desired link, the coverage probability under such setup is expressed as in (62), where $R_{0}$ can be selected uniformly at random from the transmitting BSs [19], [235] (blue points in Fig. 11), or based on the $n$th nearest serving BS policy [19], [63]. The MGF of the interference can be expressed using similar sequence steps in [4, Page 9], as

$$
\mathcal{L}_{I}(s)=\left(1-p+p \int_{r_{\text {in }}}^{r_{\text {out }}} f_{R}(u) \mathbb{E}_{g}\left(\mathrm{e}^{-s g / \mathrm{L}(u)}\right) \mathrm{d} u\right)^{N},
$$

where $s=\mathrm{TL}\left(R_{0}\right)$ and $f_{R}($.$) is the PDF of the distance from$ the reference receiver to interferers, expressed as [235]

$$
f_{R}(u)=\left\{\begin{array}{lr}
\frac{2 \pi u}{\left|\mathcal{A} \cap C_{\xi}\right|}, & r_{\text {in }} \leq u \leq r_{\max } \\
\frac{2 u}{\left|\mathcal{A} \cap C_{\xi}\right|} \cos ^{-1}\left(\frac{u^{2}+d^{2}-\xi^{2}}{2 d u}\right), & r_{\max } \leq u \leq r_{\mathrm{out}}
\end{array}\right.
$$

where $r_{\max }=\max \left(r_{\text {in }}, \xi-d\right)$.

5) Nakagami fading on the desired signal: Capturing small-scale fading with Rayleigh distribution is particularly justified in NLOS propagation environments. However, in the context of UDNs, where the transmitter-receiver distance is reduced, the likelihood of specular LOS paths increases, and the Rayleigh assumption is no longer realistic. Similar observation is considered in the context of higher-frequency bands where signal propagation is generally sensitive to LOS and NLOS paths [165], [172]. In such scenarios, Rician fading is commonly accepted to capture fading in LOS propagation [236], [237], where it can be well approximated by means of a more tractable Nakagami- $m$ distribution. Also, the Nakagami assumption can actually be seen as a gamma distribution since $X^{2}$ is gamma distributed when $X$ is Nakagami distributed, which improves analytical convenience.

We now assume that the desired link $h$ follows a gamma distribution with shape parameter $m$ and scale parameter $\theta$, (60) simplifies then as

$$
\begin{aligned}
& \mathrm{P}_{\mathrm{c}}(\mathrm{T})=\mathbb{E}_{R_{0}, I, W}\left(\frac{\Gamma\left(m, \frac{\mathrm{T}}{\theta} \mathrm{L}\left(R_{0}\right)(I+W)\right)}{\Gamma(m)}\right) \\
& \stackrel{(\mathrm{a})}{=} \mathbb{E}_{R_{0}}\left(\sum_{k=0}^{m-1} \frac{(-1)^{k}}{k !}\left[s^{k} \frac{\mathrm{d}^{k} \mathcal{L}_{I+W}(s)}{\mathrm{d} s^{k}}\right]_{s=\frac{\mathrm{T}}{\theta} \mathrm{L}\left(R_{0}\right)}\right),
\end{aligned}
$$

where (a) follows from the expansion of the upper incomplete gamma function as $\Gamma(m, x)=\Gamma(m) e^{-x} \sum_{k=0}^{m-1} \frac{x^{k}}{k !}$.

The computation of coverage probability in (76) requires then a prior evaluation of the $k$ th derivative of $\mathcal{L}_{I+W}(s)$. Assuming the interference-limited regime, i.e., $\mathcal{L}_{I+W}(s) \simeq$ $\mathcal{L}_{I}(s)$, several frameworks have been proposed in the literature to derive or approximate the $k$ th derivative of the Laplace transform of the interference. For instance, an approximation via Taylor expansions is considered in [238]. In [239]-[241], The authors proposed the use of the Faà di Bruno's formula [242], where an alternative formulation under the Bell polynomials is used in [73]. Also, a recursive-technique is proposed in [241], [243], where the expression of the $k$ th derivative is transformed to a lower triangular Toeplitz matrix with positive entries. However, reducing analysis to the interference-limited regime can be seen as less efficient in scenarios where thermal noise is a key player in the network performance, e.g., higherfrequency bands. Interestingly, an alternative framework based on the Alzer's lemma is suggested in [71], [162], which enables to derive a relatively tight approximation of coverage probability, as

$$
\mathrm{P}_{\mathrm{c}}(\mathrm{T}) \approx \mathbb{E}_{R_{0}}\left(\sum_{k=1}^{m}(-1)^{k+1}\left(\begin{array}{c}
m \\
k
\end{array}\right) \mathcal{L}_{I}(k s) \mathcal{L}_{W}(k s)\right),
$$

where $s=\frac{\beta \mathrm{T}}{\theta} \mathrm{L}\left(R_{0}\right)$ and $\beta=\Gamma(1+m)^{\frac{-1}{m}}$.

It should be noted that this framework is generally suitable whenever the desired link is experiencing a fading model of the form [238, Theorem 1], $\mathbb{P}(h>u)=$ $\sum_{n \in \mathbb{N}} \mathrm{e}^{-n u} \sum_{k \in \mathbb{N}} a_{n k} u^{k}$.

6) The factorial moment based approach: Considering the max-SINR association policy, the authors of [56], [244][246], conceived the coverage probability experienced by the typical UE $y$ w.r.t. all BSs $x \in \Psi$, as the probability that the $k$ th smallest BS in terms of SINR meets the required target $\mathrm{T}$. In other words, the typical user is in coverage if at least $k$ BSs meet the required SINR target. Formally,

$$
\mathrm{P}_{\mathrm{c}}^{(k)}(\mathrm{T})=\mathbb{P}\left(\left[\sum_{x \in \Psi} \mathbb{1}(\operatorname{SINR}(x ; y)>\mathrm{T})\right] \geq k\right) .
$$

Let's denote by $n \geq 1$, the number of BSs with SINR greater than the required threshold. Next, the authors introduced a 
key quantity of interest, namely the factorial moment measure $S_{n}(\mathrm{~T})$ of the SINR process, defined as the average number of ways that the typical UE can be associated to $n$ different BSs. Formally, it can be expressed as

$$
S_{n}(\mathrm{~T})=\mathbb{E}\left(\sum_{x_{1}, \ldots, x_{n} \in \Psi}^{\neq} \mathbb{1}\left[\bigcap_{i=1}^{n} \operatorname{SINR}\left(x_{i} ; y\right)>\mathrm{T} \mid x_{i} \in \Psi\right]\right) \text {. }
$$

Interestingly, (77) can be simplified via the famous inclusionexclusion principle [56], [244]-[246], as

$$
\mathrm{P}_{\mathrm{c}}^{(k)}(\mathrm{T})=\sum_{n=k}^{\infty}(-1)^{n-k}\left(\begin{array}{l}
n-1 \\
k-1
\end{array}\right) S_{n}(\mathrm{~T}) \text {. }
$$

The sum in (79) is actually finite since $n \mathrm{~T} /(1+\mathrm{T})$ needs to be lowered by 1 as demonstrated in [4, Proposition 6.2]. That is, (79) is simplified as

$$
\mathrm{P}_{\mathrm{c}}^{(k)}(\mathrm{T})=\sum_{n=k}^{\lceil 1 / \mathrm{T}\rceil}(-1)^{n-k}\left(\begin{array}{l}
n-1 \\
k-1
\end{array}\right) S_{n}(\mathrm{~T}) .
$$

The computation of the $k$-coverage probability in (80) requires then a prior evaluation of $S_{n}(\mathrm{~T})$ for $n \geq k$, which can be derived via higher order Campbell's theorem as in [56, Theorem 6] [246, Theorem 7]. It is worth mentioning that despite the analytical relevance of technique $\# 6$ and its ability to reflect several connectivity scenarios of the typical UE, it provides however less-tractable expressions of coverage probability and requires generally a thorough indepth knowledge of the factorial moment measure and its higher order Campbell's theorem.

7) The Plancherel-Parseval approach: For the sake of further generalization, so that the performance evaluation would not be limited to a particular fading distribution that is only valid in some operational regimes, the authors of [4], [5], [17], [18] considered the Plancherel-Parseval theorem [4, Lemma 12.2.1] to derive an exact expression of coverage probability regardless of the fading model. That is, assuming a generalized fading distribution on both the desired link and interferers, the coverage probability in (61) becomes,

$$
\begin{aligned}
\mathrm{P}_{\mathrm{c}}(\mathrm{T})=\mathbb{E}_{R_{0}}\left(\int_{-\infty}^{\infty} \mathcal{L}_{I}(2 j \pi \mathrm{L}\right. & \left.\left(R_{0}\right) \mathrm{T} s\right) \mathcal{L}_{W}\left(2 j \pi \mathrm{L}\left(R_{0}\right) \mathrm{T} s\right) \\
& \left.\times \frac{\mathcal{L}_{h}(-2 j \pi s)-1}{2 j \pi s} \mathrm{~d} s\right) .
\end{aligned}
$$

8) The Gil-Pelaez inversion approach: An alternative way to incorporate generalized fading, is by using the GilPelaez inversion theorem [247], in which the cumulative distribution function (CDF) $F_{X}$ of a random variable $X$ can be expressed based on the characteristic function $\Phi_{X}(\omega)$, as

$$
F_{X}(x)=\frac{1}{2}-\frac{1}{\pi} \int_{0}^{\infty} \frac{\operatorname{Im}\left(e^{-j \omega x} \Phi_{X}(\omega)\right)}{\omega} \mathrm{d} \omega .
$$

Relevant applications can be found in [86], [165], [212], [248], where the coverage probability in (60) is reformulated as

$$
\begin{aligned}
& \mathrm{P}_{\mathrm{c}}(\mathrm{T})=\mathbb{E}_{R_{0}, h, W}\left(\mathbb{P}_{I}\left(I \leq \frac{h}{\mathrm{TL}\left(R_{0}\right)}-W \mid R_{0}, h, W\right)\right) \\
& =\frac{1}{2}-\frac{1}{\pi} \mathbb{E}_{R_{0}}\left(\int_{0}^{\infty} \operatorname{Im}\left(\Phi_{h}\left(\frac{-\omega}{\mathrm{TL}\left(R_{0}\right)}\right) \Phi_{W}(\omega) \Phi_{I}(\omega)\right) \frac{\mathrm{d} \omega}{\omega}\right)
\end{aligned}
$$

where the characteristic function is obtained as

$$
\Phi_{I}(\omega)=\exp \left(-2 \pi \lambda \int_{\vartheta\left(R_{0}\right)}^{\infty}\left[1-\mathbb{E}_{g}\left(\exp \left(\frac{j \omega g}{\mathrm{~L}(u)}\right)\right)\right] u \mathrm{~d} u\right),
$$

such that $\vartheta($.$) is the function considered in technique \# 1$.

Interestingly, the Gil-Pelaez technique can also be leveraged to invert the $j w$-moment of the conditional success probability in order to derive important metrics of interest under the spatio-temporal modeling of traffic such as mean delay and peak AoI [201], [202], [208].

9) The Laplace transform inversion approach: Another inversion technique to derive the PDF of a random variable $X$, is by considering the Fourier inversion theorem, also known as the Laplace transform inversion [19], [55], [250], [288], the characteristic function inversion [89], [251], or even the MGF inversion [237]. Generally, the PDF $f_{X}($.$) of X$ is obtained via the Bromwich contour inversion integral, as

$$
f_{X}(y)=\mathcal{L}^{-1}\left\{\mathcal{L}_{X}(s)\right\}=\frac{1}{2 \pi j} \int_{\gamma-j \infty}^{\gamma+j \infty} \mathcal{L}_{X}(s) \mathrm{e}^{y s} \mathrm{~d} s,
$$

where $\gamma$ is a real constant such as the contour of integration runs from $\gamma-j \infty$ to $\gamma+j \infty$ along a straight line and lies to the right of all the singularities of $\mathcal{L}_{X}($.$) .$

As for the CDF of $X$, it can be derived equivalently as

$$
F_{X}(x)=\int_{0}^{x} f_{X}(y) \mathrm{d} y=\mathcal{L}^{-1}\left\{\frac{\mathcal{L}_{X}(s)}{s}\right\}(x) .
$$

That is , the coverage probability in (83), simplifies then as

$$
\mathrm{P}_{\mathrm{c}}(\mathrm{T})=\mathbb{E}_{R_{0}, h, W}\left(\mathcal{L}^{-1}\left\{\frac{\mathcal{L}_{I}(s)}{s}\right\}\left(\frac{h}{\mathrm{TL}\left(R_{0}\right)}-W\right)\right) .
$$

Similarly to previous inversion techniques, this approach derives exact expressions of coverage probability under generalized fading distribution, but requires involved analysis with limited design insights [246]. A more flexible version is to resort to the characteristic function inversion enabling to avoid contour integration as illustrated in [89], [251].

10) The interference approximation approach: An alternative way to derive the PDF of the interference without resorting to previous less tractable inversion techniques, is to approximate the interference behavior [25]. Two main approaches are considered in the literature. The first one, is by considering the interference contribution from only some specific transmitters based on the adopted association policy. For example, the $k$ dominant interferers in terms of the received power are considered when assuming max-SINR association criterion [48], while the $k$-nearest interferers are considered in the case of the nearest [178] or the $n$th nearest neighbor association policy [195]. This approach enables actually to derive an upper bound of coverage probability, where bound accuracy increases with increasing path loss exponent to justify ignoring the contribution of distant interferers [25]. The second approach is by approximating the distribution of the other-cell interference via well-known distributions with parameters fitting the essential physical parameters that affect interference. Typically, the authors of [252] showed that the interference behavior can be captured by the family of $\alpha$ stable distributions, while in [253], interference distribution is approximated via gamma distribution. 
TABLE VI

KEY ANALYTICAL TECHNIQUES USED IN WIRELESS NETWORKS PERFORMANCE EVALUATION UNDER THE PPP/BPP ABSTRACTION

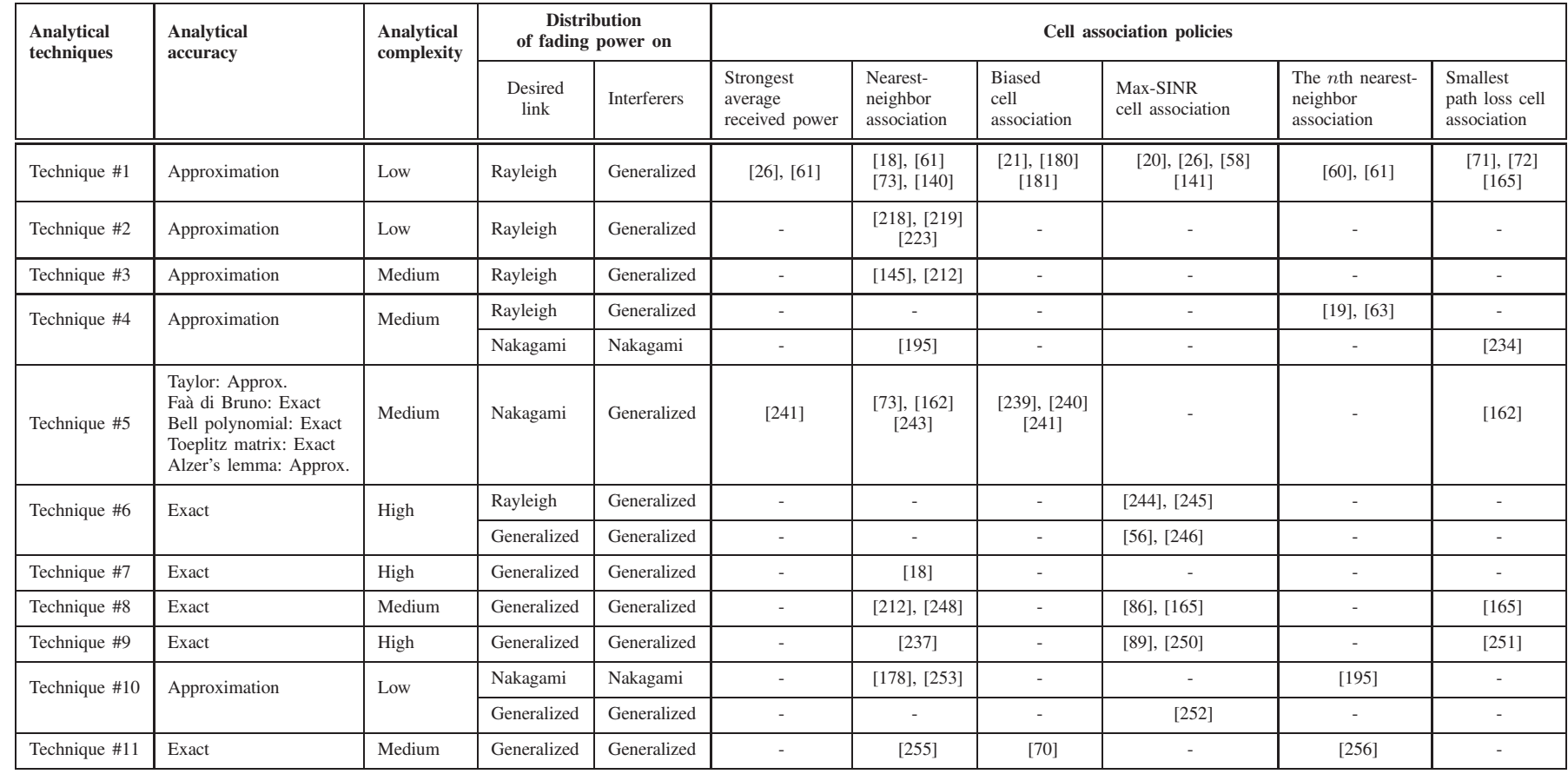

11) MGF-based average rate: As has been discussed in (48), average ergodic rate is commonly mapped to coverage probability via integration over the positive real axis (Fig. 10). Such an approach reduces the use of time-consuming simulations, but requires however the computation of multi-fold numerical integral. An alternative approach is proposed in [254] to derive the average ergodic rate by considering general fading distributions and without necessarily going through the coverage probability expression. A qualitative and quantitative comparisons of the MGF-based framework with the coveragebased conventional approach can be found in [70]. Typically, using [254, Lemma 1], the average ergodic rate in (47) can be simplified as

$$
\begin{aligned}
& \tau=\mathbb{E}_{R_{0}, W}\left(\mathbb{E}\left(\log \left(1+\frac{\frac{h}{W \mathrm{~L}\left(R_{0}\right)}}{1+\frac{I}{W}}\right) \mid R_{0}, W\right)\right) \\
& =\mathbb{E}_{R_{0}}\left(\int_{0}^{\infty} \mathcal{L}_{W}(s) \frac{\mathcal{L}_{I}(s)\left(1-\mathcal{L}_{h}\left(\frac{s}{\mathrm{~L}\left(R_{0}\right)}\right)\right)}{s} \mathrm{~d} s\right) .
\end{aligned}
$$

Next, the framework was widely adopted in the literature. For instance, the work in [255] evaluated the uplink average ergodic rate when considering representative scheduling schemes in terms of performance and implementation complexity. In [256], the downlink average ergodic rate is investigated under the scenario of multiple cell association in UDNs environment.

Table VI summarizes the eleven techniques commonly used in the literature to evaluate main performance metrics of wireless networks under the PPP/BPP abstraction. Also, we examined their mapping with various association schemes considered in the SG literature, as well as illustrated the required fading model for each technique.

\section{Stochastic Geometry and Promising RAN ARCHITECTURES FOR 5G/B5G}

In this section, we will review key aspects and challenges of emerging RAN architectures for $5 \mathrm{G} / \mathrm{B} 5 \mathrm{G}$, and subsequently outline major state-of-the-art contributions which, based on $\mathrm{SG}$, can evaluate the key properties of these promising RAN architectures.

\section{A. Terrestrial Heterogeneous Networks}

Adding new macro BSs in a homogeneous and regular fashion is typically constrained by increased deployment costs, in addition to heterogeneity in site location availability and users demand. An alternative strategy is to consider the deployment of HetNets, where several classes of low-power and low-cost nodes are deployed in poorly covered small areas or traffic hotspots, overlaid within macro BSs.

The key aspects of a generative SG model consist of $K$ overlaid tiers of BSs, where the BSs of each tier are characterized by some distinctive marks (e.g., transmit power, connectivity threshold, BS density, backhaul type) and the locations of each tier nodes are modeled with a specific PP. Typically, a regular or repulsive PP for macro BSs and clustering PPs for lowpower cells [66], [67]. For instance, the downlink SINR of HetNets is evaluated under various BS association policies and fading models in [20], [21], [26], [58], [245], [256]. The uplink analysis of HetNets is studied in [152]-[154]. Also, given the heterogeneous attributes of BS transmit power in HetNets, the typical UE may be associated to different BSs in uplink and downlink, i.e., DUDA. Further analysis of DUDA in the context of HD HetNets can be found in [156]-[158], while DUDA in the context of IBFD HetNets is given in [182] and the references therein. One important use case of small cells 
in HetNets is to support overloaded macro cells. That is, the impact of CRE on the performance of HetNets is assessed in [141], [180], [181]. Last but not least, the question of mobility is also crucial in HetNets where it is generally governed by several tradeoffs. For example, a mobile UE in HetNets will suffer from frequent handovers, which may increase call drop rate and service delays. However, adding more low-power cells increases the average number of lightly loaded BSs and then reduces interference [257], which can reduce handover failure rate. In all cases, incorporating mobility is very challenging given the complex behavior of users movement [183], [184], [193]. Further analysis of mobility-aware HetNets can be found in [186], [187], [189]-[191].

\section{B. Non-Terrestrial Networks}

A promising frontier for terrestrial HetNets is by extending their deployment to the sky via UAVs as aerial BSs [34], [258]. In fact, given their distinctive features, UAVs can be quickly deployed to support coverage in isolated regions and capacity of terrestrial HetNets during flash crowded events. However, despite the benefits of UAVs as flying nodes, several new challenges are introduced. Among them, the AtG propagation model, which is no longer similar to popular terrestrial models due to UAVs operational altitude and 3D mobility. Typically, the widely used AtG model is the one proposed in [173] based on a modified sigmoid function. A comprehensive survey about channel modeling for UAVassisted communications can be found in [174]. Also, given their technical constraints combined with ground UEs QoS requirements, optimal placement of UAVs is another challenging task, which may include UAVs trajectory optimization [259], altitude optimization [173], [260], flight time optimization [259], [261], and UAVs density optimization [262].

With the introduction of a new research area based on SG, spatial locations of nodes in UAV-aided wireless networks are totally or partially modeled as randomly distributed according to a PP. This approach is followed in [259], where a UAV flies sequentially over several finite fields of PPP terrestrial sensors to collect IoT big data. The results indicate that the mean square error of the mission duration can be minimized by carefully adjusting the number of sensor fields, the area of each field, the hover locations, the hover time at each location, and the flight path between hover locations. In [262], the authors investigated the performance of a vertical HetNet composed of two PPP layers of ground and aerial BSs, where the rationale is to capture the impact of UAVs altitude and density on coverage enhancement and on-demand capacity boost of ground UEs. The authors of [263] evaluate how the altitude of a single UAV operating as BS, can impact the coverage and rate of two types of ground UEs: downlink UEs and D2D UEs. Next, it has been found that in order to provide coverage for all downlink UEs, UAV need to move around the overall area of interest in such a way that the number of stopping points increases monotonically with the density of downlink UEs. In such a context, handover needs actually to be taken into careful consideration since UAV mobility can improve the coverage of one UE but it is likely to affect the coverage of another. In [195], a finite network of aerial BSs whose locations are modeled as a uniform BPP is considered to support coverage at a reference ground UE. Assuming independent Nakagami-m fading for all wireless links, a general expression of the downlink coverage probability is derived by using the analytical techniques \#4 and \#5 and approximations were next made using the dominant interferer-based approach in technique \#10.

In [264], UAVs are aimed to assist public safety networks, where the location of ground BSs surviving after a natural disaster are modeled as an independent thinned PPP, while UAVs form a PCP around the locations of destroyed BSs to replace them. A Key outcome suggest that efficient coverage by the emergency network can be achieved by adjusting the number of UAVs per cluster, the flight altitude, and the transmission power ratio between aerial and ground BSs. Sometimes and due to their limited storage and processing capabilities, UAVs are required to operate as mobile aerial RNs for ground nodes. In particular, the authors of [265] considered a setup where a thinned part from PPP distributed UAVs are used as airborne DF relays to support mmWave communication of a terrestrial legitimate UE, and the other part of the UAVs are used to jam the channel quality of PPP distributed terrestrial malicious eavesdroppers. In particular, it has been shown that more secure communications can be achieved by making a convenient tradeoff between system-level parameters of relaying and jamming UAVs, which actually corresponds to tuning the thinning probability.

In [261], the authors adopted a similar abstraction of vertical HetNet, as in [262], to address the question of the limited energy resources when using UAVs as a BS, as this leads to restricted flight times, and therefore forces UAVs to regularly interrupt their operations to recharge or swap their batteries. One key outcome is that battery quality (quantified by the charging speed) and the density of charging stations play a supplementary role in minimizing UAV interruption time and then in achieving the required QoS for terrestrial UEs. Alternatively, the authors of [266] considered tethered UAVs to alleviate the limitations of conventional untethered UAVs in terms of battery autonomy and backhaul capacity between aerial and ground BSs. Accordingly, since untethered UAVs are limited by the need to remain sufficiently close to their backhaul-BS, which limits their ability to serve larger areas, the study's results show that tethered UAVs can outperform their untethered counterparts in terms of the end-to-end coverage probability, defined as the joint probability that the SNR of out-of-band backhaul and access link are greater than a predefined threshold. However, in realistic scenarios, tethered UAVs are also limited by the length of the tether and the likelihood that the ground backhaul-BS will be located away from UEs hotspot, which can significantly reduce the performance of tethered UAVs.

In [267], UAVs are abstracted as aerial UEs. The authors have derived the uplink EE coverage under a setup where two classes of PPP terrestrial and aerial UEs are served by ground BSs. It has been shown that an aggregation of systemlevel parameters of aerial UEs (through the aerial priority bias) needs to meet some transcendental constraint, based on 
TABLE VII

KEY MODELING CHOICES IN SG-BASED MODELING AND ANALYSIS OF UAV-ASSISTED WIRELESS NETWORKS

\begin{tabular}{|l|l|l|l|l|l|l|l|}
\hline Reference & Terrestrial nodes & Aerial nodes & UAV function & Performance metrics & Association policy & Analytical techniques & Optimization task \\
\hline \hline$[259]$ & BPP & One single UAV & Data aggregator & Success probability & Smallest path loss & Techniques \#4 and \#5 & UAV trajectory and hovering time \\
\hline$[262]$ & PPP & PPP & Airborne BS & $\begin{array}{l}\text { Coverage probability, } \\
\text { ergodic rate }\end{array}$ & Smallest path loss & Technique \#5 \\
\hline$[263]$ & BPP, PPP & One single UAV & Airborne BS & Coverage probability & Smallest path loss & Technique \#4 \\
\hline$[195]$ & One single UE & BPP & Airborne BS & Coverage probability & Smallest path loss & Techniques \#4, \#5, and \#10 & UAV density and altitude \\
\hline$[264]$ & PPP & PCP & $\begin{array}{l}\text { Emergency } \\
\text { Airborne BS }\end{array}$ & Coverage probability & Smallest path loss & Technique \#1 \\
\hline$[265]$ & One single BS & PPP & $\begin{array}{l}\text { Airborne } \\
\text { DF Relay }\end{array}$ & Secrecy outage & Smallest path loss & Technique \#5 \\
\hline$[261]$ & PPP, PCP & PPP & Airborne BS & Coverage probability & Smallest path loss & Technique \#5 \\
\hline$[266]$ & BPP & One single UAV & $\begin{array}{l}\text { Tethered } \\
\text { airborne BS }\end{array}$ & Coverage probability & Smallest path loss & Technique \#4 \\
\hline$[267]$ & PPP & PPP & Airborne UE & Uplink energy efficiency & Biased cell association & Technique \#1 \\
\hline
\end{tabular}

the principal branch of the Lambert $\mathrm{W}$ function, to mitigate interference from aerial UEs and enhance the uplink EE coverage of ground UEs. It is worth highlighting that despite the expected advantages of UAVs when exploited as aerial UEs in communication networks. They can however adversely affect the performance of terrestrial UEs, which are usually assigned more mission-critical roles than UAVs (e.g. monetary transactions, health care services). As an illustration, we plot in Fig. 12 the fluctuations of the uplink terrestrial coverage EE, i.e., the probability that the uplink EE of terrestrial UEs is greater than a predefined threshold, as a function of aerial UEs parameters [267].

Table VII summarizes the key modeling choices made in previous relevant research papers on the applications of SG in the modeling and analysis of UAV-assisted wireless networks. In particular, it can be observed that since UAVs can offer an extremely agile deployment allowing LOS transmissions to be established with ground stations (e.g. UEs, BS, sensors), Nakagami- $m$ fading is considered to capture such AtG links and then the association policy based on the smallest path loss and the analytical technique \#5 are generally the most widely adopted modeling choices in the SG-based literature.

\section{UDNs via Infrastructure Densification}

Infrastructure densification is envisioned as the workhorse for ubiquitous coverage and capacity improvement in 5G/B5G networks [268]-[270]. It can generally be realized by adding new transmitters in the area of interest, which may be in the form of new BSs or distributed antennas from the existing ones. Many experts also consider some spatial diversity technologies such as relays, and D2D communications, as a form of densification since they allow decentralized opportunistic short-range communication [268]. In the following, we will discuss the main technologies competing in terms of infrastructure densification in HetNets, as well as the key state-ofthe-art contributions based on SG.

1) Small cells: Adding small cells is a common way to emerge from HetNets to UDNs. Several challenges are, however, brought into analysis. For instance, the question of association policy is a key concern, where UEs can access

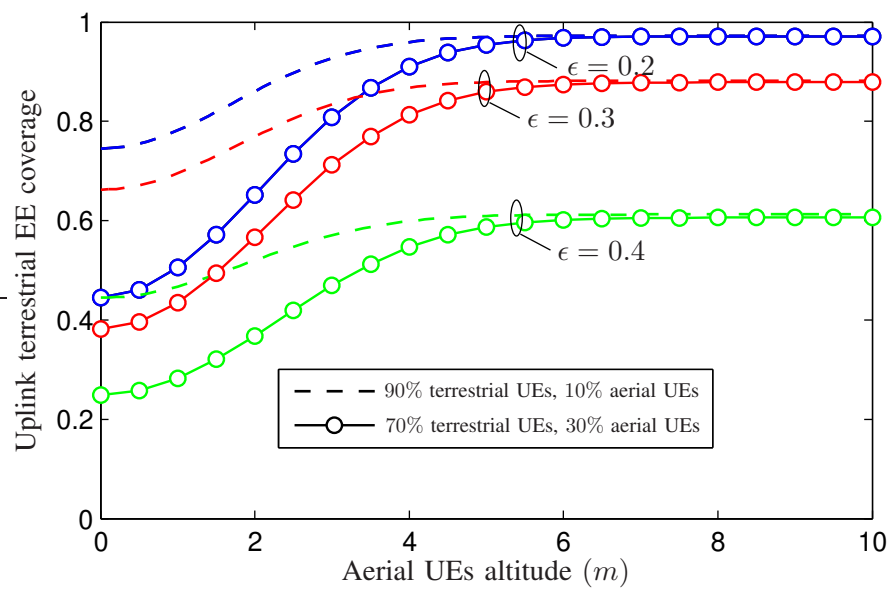

Fig. 12. Mean load approximation of the uplink terrestrial EE coverage for the threshold $10 \mathrm{bps} / \mathrm{W}$ as a function of aerial UEs altitude, the power control coefficient $\epsilon$, and the average density of terrestrial/aerial UEs per BS. Numerical parameters: frequency $2.1 \mathrm{GHz}$, path loss exponent 4 , and the total effective uplink bandwidth $5 \mathrm{MHz}$.

small cells without any logical restriction, namely open access, and hence the need to judiciously adjust cells load via CRE [180], [181] and interference via intercell interference coordination (ICIC) [271]. Also, access to small cells can exclusively be given to some specific UEs belonging to a closed subscriber group (CSG), namly closed access, or consider an hybrid access scheme, in which some additional UEs not registered in the CSG can also access the cell along with registered UEs [274]. Typical challenges may also include, the scaling law of network performance with infrastructure density [36], [72], [73], [168], [170], [171], [177], [269] and qualitative and quantitative comparison between adding new cells, using multi-antenna transmissions, or increasing macro cells storage capacity [272], [273]. More discussions about other representative challenges are given in [268]-[270].

2) Infrastructure sharing: A new viable business model for infrastructure densification is by allowing concurrent operators share their mutual infrastructure [275]. This paradigm is expected to lower the time to market of each operator and reduce costs by an average of up to $40 \%$ and $15 \%$ in terms of capital expenditure (CAPEX) and operational expenditure 
(OPEX) costs, respectively [276]. Questions related to the optimal sharing strategy between operators, are actually the main concern of research studies. Typically, two extreme variants of infrastructure sharing are considered: Passive sharing, wherein operators can only share site location and common operation costs, while each operator installs and maintains its own equipment, and active sharing wherein operators share their network physical infrastructure and have access to it on the basis of an agreed resource allocation strategy.

Based on the tractability of SG, several mathematical frameworks and PP models have been investigated to quantify the benefits of infrastructure sharing. For instance, the authors of [136] evaluated the goodness-of-fit of some realistic sharedinfrastructure networks with various aggregative PP models, where they revealed that LGCP can serve as a universal model to fit realistic multi-network empirical data. In [74], infrastructure sharing is evaluated under a setup of one buyer operator and multiple seller operators, where the aim of the study is to define an optimal buying strategy to meet a target QoS requirement with reduced costs. In [75], the authors derived the potential SE required per tenant and formulated an optimization problem to identify the optimal transmit power and spectrum to guarantee the minimum potential SE required by each tenant. In [76]-[78], spectrum sharing between several operators is studied, particularly at mmWave frequencies where antenna beamforming, transmissions sensitivity to blockages, and operators cooperation, can help to mitigate inter-operator interference [277]. In [79]-[81] both spectrum and infrastructure sharing are investigated.

3) Multi-hop relays: One practical limitation of densifying HetNets via fully-functioning new cells is the complexity of provisioning all new cells with a dedicated wired backhaul connection. One proposed solution is to consider the deployment of RNs between BSs and cell-edge UEs [283]. That is, several relaying protocols have been investigated by the research community. For instance, amplify-and-forward (AF) $\mathrm{RN}$, decode-and-forward (DF) RN, also known as L2 relay, and L3 RN, envisioned to support almost similar capabilities as small cells but without the need of a wired backhaul connection. Several use cases of RNs are assessed in the literature. For instance, RN with IBFD capability is surveyed in [284]. Cooperative RNs to create spatial diversity are investigated in [285]. In [286], cooperative RNs can be equipped with buffers to store received packets and resend them when optimal connectivity conditions are met.

Using methods from SG, several research works have considered analytical performance evaluation of relay-aided wireless networks. For instance, a novel analytical framework for the analysis of outage probability in the regime of high SNR and low BS density is proposed in [287]. The paradigm of SWIPT with cooperative relaying is investigated in [288], [289]. Quantifying the performance gain achieved when using RNs in HetNets is studied in [290]. In [291], a flexible cell association scheme is proposed, where some bias coefficients are introduced to prioritize the association of the typical UE with single- or multi-hop links, and then optimize the overall end-to-end coverage and rate. Interestingly, the performance of IBFD relay-aided cellular networks where BSs and RNs are equipped with MIMO antennas, is investigated in [292].

4) Device-to-device communications: Cooperative communications via fixed terminal relaying can bring substantial improvements in wireless networks [285]. However, with the drastic growth in UE's density and their unpredictable complex movement [183], the paradigm of cooperative communications via D2D communications is considered. Comprehensive surveys about D2D communications underlaying cellular networks can be found in [293], [294].

SG has been extensively explored in modeling and analysis of D2D communications. For instance, the crucial question of spectrum sharing in D2D communications is investigated in [295], [296]. Also, since neighbor D2D UEs are more likely to be in prominent LOS transmissions, the authors of [297] evaluated network performance under Rician small-scale fading. Due to many technical challenges when deploying D2D communications in licensed bands, traffic offloading via D2D in unlicensed bands is considered in [298]. The tendency of D2D UEs towards clustering is captured via PCP in [160], [161], where coverage probability and ASE are next derived under several content availability scenarios. Last but not least, uplink analysis of D2D communications is provided in [299], the benefits of combining IBFD with the merit of underlaid D2D communications are evaluated in [300], the impact of massive MIMO on reducing D2D-to-cellular interference is studied in [282], and the performance of D2D communications when considering aerial access points is available in [263].

\section{Cloud Radio Access Networks}

Albeit the expected benefits of infrastructure densification, there are still many notable limitations. Among them, the increasing CAPEX and OPEX costs with densification [50]. Also, interference is expected to be more critical, which demands an aggressive frequency reuse. The question of mobility is also crucial due to reduced cells size and heterogeneity in neighboring cells, which requires proper load balancing and smooth handover schemes. An innovative paradigm to address such challenges, is to consider C-RAN architecture, in which the baseband units (BBUs), responsible of scheduling and data processing, are separated from radio units, and pooled farther away in a centralized data center equipped with the potential of cloud computing. Remote radio heads (RRHs) are connected to BBUs via a dedicated high-speed and low-latency links, e.g., radio over fiber, namely the fronthaul link. C-RAN can therefore manage simultaneously the BBU processing of a large geographical zone, which reduces costs (e.g., about $15 \%$ in CAPEX and 50\% in OPEX [50]), facilitates load balancing, and enables the use of ICIC techniques and coordinated multipoint (CoMP) transmission and reception, considered as potential solutions in C-RAN [51]. One important variant of C-RAN is distributed antenna system (DAS), in which BS antennas and inherent radio frequency (RF) components are deployed far away from the BS to form a distributed antenna array, while the central intelligence is kept at the BS level. A typical application of DAS is the reinforcement of indoor coverage as aimed by the baseline work in [278].

Using tools from SG, the dynamics on the location of nodes in a C-RAN architecture, are captured for analytical evaluation 
of network performance. For instance, a model of 4-layers of nodes (UEs, RRHs, backhaul nodes, and data centers), modeled by independent PPPs is considered in [301], where by assuming various representative costs (cost of nodes, processing, and backhaul technology), the authors demonstrated that C-RAN based architectures can reduce costs by at least $10 \%$. In [302], the performance of a C-RAN with multiple antenna RRHs is evaluated when assuming three degrees of collaboration between RRHs and their associated BS. In [303], the authors considered MHPP II to capture the repulsion behavior in RRHs location. Interestingly, the contributions of some emerging techniques in the context of C-RAN is also studied. Representative works can be found in [304] for CRAN with ICIC techniques, in [305] for C-RAN as an enabler for CoMP protocols, in [306] for IBFD transmissions, and in [307] for NOMA-based communications. As for the DAS variant of C-RAN, outage probability is investigated in [279], while spectral efficiency is studied in [280], [281].

\section{E. Virtualized Radio Access Networks}

Increased network densification, as well as the use of CoMP and ICIC techniques in a C-RAN architecture, are expected to boost the overall network performance. However, they can amplify the signaling and control overhead, which is expected to generate a critical burden at the fronthaul level. One promising approach to alleviate the fronthaul bottleneck is by splitting the control plane (C-plane) and the user plane (Uplane) of the radio link via $R A N$ virtualization. This capability is particularly supported by the software-defined networking (SDN) [308] where U-plane (message forwarding) is deployed in a decentralized fashion, while C-plane (control and radio resource management) is centralized in a controller. Typically, the C-plane can be provided by high-power nodes operating at sub-6 GHz bands to guarantee large coverage and efficient mobility schemes, while the U-plane can be provided by low-power nodes, namely phantom cells, operating at higherfrequency bands [309], [310]. Such low/high-frequency bands operation helps actually to pave the way for joint URLLC and eMBB communications [311], which generally requires a prior combining of the C-RAN paradigm with HetNets, also known as H-CRAN [312].

Based on the tractability of SG, several recent works have investigated the performance gain under the setup of $\mathrm{C}$ plane/U-plane split architecture. For instance, the authors of [313] evaluated EE improvements under the phantom cell paradigm as compared to a macro-only deployment. In [314], the authors studied offloading of the macrocellular layer through small cells CoMP transmissions in a virtualized RAN architecture. In [315], a tractable mobility-aware model is considered to quantify the expected performance gain with C-plane/U-plane split. Further extensions of the analysis are considered in [316] by taking into account mmWave sensitivity to LOS and NLOS transmissions, and in [317] by considering UAV-aided cellular networks.

\section{F. Fog Radio Access Networks}

Another promising paradigm to alleviate the fronthaul burden in H-CRAN is F-RAN architecture, in which a consider- able fraction of the cloud is deployed in close proximity to UEs, which can be done through endowing edge terminals or third-party entities (e.g., parks, shopping centers) with computing and storage capabilities [318]. There are generally two typical applications of such promising paradigm. i) Storing and computing capabilities, wherein computation-intensive tasks are processed at the level of nearby fog servers and the result will be forwarded back to end UEs, which enables using the released memory space at UEs to process other services, and then enhance users QoE. ii) Content delivery and caching, wherein close fog servers, also known in this context as helper nodes, are endowed with high capacity caches in order to proactively cache popular internet content requested by end UEs [35].

Using tools from SG, most literature works on the analysis of F-RAN architectures, are typically focused on quantifying the benefits of the caching capability of helper nodes when assuming a network with limited backhaul [273] or fronthaul link capacity [319]. Typically, the SG generative setup is to consider the location of helper nodes as modeled according to some PP (e.g., PPP [273], $\beta$-GPP [319], MHPP II [320], PCP [161]), each node has a finite cache capacity, wherein files are placed according to some popularity distribution function (e.g., Zipf), and each cached file requires a minimum bitrate to meet the requirements of users QoE. That is, the performance of a cache-enabled network is quantified by the average delivery rate, defined as the probability that the typical UE can receive a downlink rate greater than the file bitrate threshold, and also the requested file can be found in the local cache of the tagged helper node. Otherwise, the file will be requested from the core network, and the average delivery rate of files will be constrained by the backhaul/fronthaul link capacity.

Accordingly, two lines of research are adopted in the literature. The first, is related to the content placement strategy, in which the problem can be stated as, how should we place the files in the helper caches to optimize some performance metrics (e.g., the hit probability defined as the probability that the typical UE may find the requested file at the tagged helper node)? Representative works can be found in [321] for singletier cellular networks, in [320] for D2D communications, and in [322] for content placement policy in large-scale HetNets. The second direction of research is related to the optimal delivery strategy, wherein the analysis is focused on how to deliver the cache content at the user request in order to boost some performance metrics (e.g., the average delivery rate). For instance, the average delivery rate is considered in [273] to evaluate the question of adding more BSs or increasing the caching capability of already deployed BSs. In [323], the content delivery protocol is studied in a HetNet scenario where the typical UE can request content from the nearest BS, $\mathrm{RN}$, or cache-enabled UE. Interestingly, the joint analysis of placement and delivery techniques is brought in [324], [325].

\section{Stochastic Geometry And Key 5G/B5G EMERGING TECHNOLOGIES}

In this section, we will review modeling challenges to capture the properties of 5G/B5G key emerging technologies, 
and highlight the penetration degree of SG in modeling and analysis of their fundamental attributes.

\section{A. Stochastic Geometry for Communications in Higher Fre- quency Bands}

With the severe spectrum scarcity in commercial wireless networks running generally at sub- $6 \mathrm{GHz}$ frequency bands, researchers are steering new opportunities in higher frequency bands to conceive a sufficiently higher bandwidth and hence meet the increased data rate requirements for eMBB services [269]. In the following, we will outline the key aspects and challenges of using SG to evaluate communications in some key higher frequency bands.

1) mmWave communications: With wavelengths from 1 to $10 \mathrm{~mm}$ and frequency range from 30 to $300 \mathrm{GHz}, \mathrm{mmWave}$ combined with advances in integrated circuit technologies enable to concentrate tens of miniaturized and high gain antennas in small areas, which permits directional beamforming alignment and enables to compensate for the excessively high propagation loss in higher frequencies [29], [71], [172]. In this way, several changes need to be considered w.r.t. conventional mathematical SG frameworks available for modeling and analysis of $\mu$ Wave wireless networks. Typically, i) nodes are equipped with directional antennas such that the antenna gain is maximized when the steering angle is inside a given main lobe width [164], [165], ii) the vulnerability of mmWaves to blockages is captured by considering LOS and NLOS transmissions in addition eventually to an outage state, in which the path loss is approximately infinite [86], [165], [326], and iii) the primacy of the thermal noise w.r.t. the interference in mmWave communications [326], is captured by assuming the noise-limited regime, i.e., SINR $\simeq$ SNR. This could, for example, be omitted in the case of UDNs where the high density of transmitters is likely to justify the prevalence of interference over thermal noise [71].

In addition to the previous considerations to be taken into account in the analysis of mmWave wireless networks, most of the existing literature based on SG and its inherent PP theory requires the inclusion of specific assumptions to ensure tractability. For instance, i) the distribution of random shapes incorporating blockages is assumed to be motion-invariant. This is well captured by the Boolean blockages scheme in which the planar centers of the blockages are distributed according to the PPP, while the measures of length, width, height, and orientation are i. i. d. according to a given PDF [166]. ii) mmWave is very sensitive to blockages such that large-scale reflections are generally ignored for mathematical convenience. iii) Another key assumption is that the blockages occur on each transmit-receive link independently, implying that the number of blockages on different transmit-receive links is independent. This assumption enables estimating that a particular area around the typical UE contains only LOS BSs with a decreasing exponential probability such that its parameters are fitted from the propagation environment, e.g., frequency, density, and blockages dimensions [71], [166], [172].

Based on SG, several representative research works are considered in the literature. For instance, a generative analytical framework is suggested in [71] to derive SIR distribution and average rate in a single-tier mmWave UDN (that justifies the SIR regime). The analysis is next simplified by capturing blockages effect via an approximate LOS step function (see Table V), which enhances the computation speed but at the cost of SINR distribution errors. In [165], the previous framework is further generalized by considering an outage state of the blockage model, with emphasis on the noiselimited regime. Based on the displacement theorem to absorb the shadowing effect, results reveal that expanding the outage state can reduce coverage, but at the same time it will reduce interference from outage cells. On the other hand, it has been shown that if the average cell radius is no more than $50 \mathrm{~m}$, the probability of LOS will be greater than $80 \%$, which will not only improve the throughput of mmWave over $\mu$ Wave, but also the coverage. The obtained theoretical results are also extended by taking into account beamforming alignment errors [43] and a multi-tier mmWave cellular deployment.

As noted in the previous works, densification with small cells is essential for mmWave networks to achieve acceptable coverage and throughput. However, this presents a major challenge for the backhaul network, given the complexity of bringing the backhaul to every new cell in urban areas and also given the enormous rates resulting from mmWave bandwidths of the order of GHz. In this way, the authors of [164], [327] propose to exploit the interference isolation provided by the narrow directional mmWave beams so that a wired link can provide the backhaul for some new cells via mmWave beams. In particular, [164] evaluated self-backhauling concept wherein BSs are PPP-distributed into three classes: $\mu$ Wave BSs, mmWave BSs with wired backhaul, and mmWave BSs with wireless backhaul to those BSs with wired backhaul. The displacement theorem and Laplace inversion technique are next leveraged to derive the SNR/SINR and rate coverage assuming association in access and backhaul links. For mathematical convenience, an approximate blockage model is adopted such that all adjacent BSs to a given UE were treated as LOS and BSs beyond a given distance were neglected. In [327], the authors considered a C-RAN abstraction and examined the feasibility of bringing wireless fronthaul to distributed aerial units by means of mmWave beams. The outage probability is next derived using technique \#5 that allows an upper bound based on Alzer's lemma. Results show that UAVs need to tune their altitude according to the considered frequency (mmWave or $\mu$ Wave) to ensure an acceptable coverage.

Furthermore, low-power mmWave small cells can be coupled with high-power BSs at sub- $6 \mathrm{GHz}$ such that the former provide increased data throughput, while the latter ensure wide coverage and efficient mobility systems. In such a context, [328] approximated the average spectral efficiency for a DUDA scheme under UDN configuration such that $\mu$ Wave macrocells are superimposed by mmWave small cells. In particular, power control is ignored and Rayleigh fading is adopted for enhanced tractability. Also, blockages are supposed to be impenetrable so that the receiver (in uplink or downlink) must be in LOS with transmitters to detect any signal. A key outcome of this work is an optimization 
TABLE VIII

KEY MODELING CHOICES OF SG-BASED MODELING AND ANALYSIS OF MMWAVE WIRELESS NETWORKS

\begin{tabular}{|c|c|c|c|c|c|c|c|}
\hline References & Technologies & Small-scale fading & Shadowing & Blockages model & Association policy & Analytical technique & Performance Metrics \\
\hline [71] & mmWave, UDNs & Nakagami & Ignored & $\begin{array}{l}\text { Decreasing exponential function, } \\
\text { estimated by a step function }\end{array}$ & The smallest path loss & Technique \#5 & SIR distribution and ergodic rate \\
\hline [165] & $\begin{array}{l}\text { mmWave, single-tier, and multi-tier } \\
\text { networks }\end{array}$ & Ignored & Log-Normal & $\begin{array}{l}\text { Decreasing exponential function, } \\
\text { estimated by a step function }\end{array}$ & $\begin{array}{l}\text { The smallest path loss } \\
\& \text { the maximum } \\
\text { instantaneous power }\end{array}$ & $\begin{array}{l}\text { Technique \#1 after recurrent } \\
\text { use of the displacement theorem }\end{array}$ & $\begin{array}{l}\text { SNR/SINR distribution and ergodic } \\
\text { rate }\end{array}$ \\
\hline [164] & $\mu$ Wave, mmWave, and backhauling & Ignored & Log-Normal & $\begin{array}{l}\text { LOS ball under the setup } \\
\text { of impenetrable blockages }\end{array}$ & The smallest path loss & $\begin{array}{l}\text { Technique \#1 and \#9 after recurrent } \\
\text { use of the displacement theorem }\end{array}$ & SNR/SINR and rate distribution \\
\hline [327] & mmWave, fronthauling, and UAV & Nakagami & Ignored & Decreasing exponential function & The smallest path loss & Technique \#5 & SINR distribution \\
\hline [328] & $\mu$ Wave, mmWave, UDNs, and DUDA & Rayleigh & Ignored & $\begin{array}{l}\text { LOS ball under the setup } \\
\text { of impenetrable blockages }\end{array}$ & $\begin{array}{l}\text { The Strongest average } \\
\text { received power }\end{array}$ & Technique \#1 & Average spectral efficiency \\
\hline [329] & $\mu$ Wave, mmWave, and DUDA & Rayleigh & Ignored & $\begin{array}{l}\text { LOS ball under the setup } \\
\text { of impenetrable blockages }\end{array}$ & $\begin{array}{l}\text { The Biased cell } \\
\text { association }\end{array}$ & $\begin{array}{l}\text { Technique \#1 after recurrent } \\
\text { use of the displacement theorem }\end{array}$ & SNR/SINR and rate distribution \\
\hline
\end{tabular}

problem enabling to maximize the downlink spectral efficiency under the constraint of a minimum required uplink data rate as a function of the $\mu$ Wave/mmWave bandwidth and BS/UE transmit power and density. In [329], a similar system model is adopted under the biased cell association scheme. With the diverse propagation patterns between $\mu$ Wave and mmWave and the wide imbalance in their bandwidths, the primary challenge of the study was to identify the optimal association bias allowing to maximize coverage and rate for the DUDA scheme.

Table VIII summarizes the key modeling choices of some relevant studies in SG-based modeling and analysis of mmWave cellular networks.

2) Terahertz communications: Compared to mmWaves, directional beamforming alignment in terahertz frequencies (from $300 \mathrm{GHz}$ to $3 \mathrm{THz}$ and wavelengths from $100 \mu \mathrm{m}$ to 1 $\mathrm{mm}$ ) is considerably more feasible due to shorter wavelength, which suggests roughly the same system model changes as those previously discussed in mmWave. However, additional key changes to the system model need to be considered in the case of $\mathrm{THz}$ communications [30]: First, since $\mathrm{THz}$ has narrower beams as well as being more sensitive to blockages (objects, human bodies), fast fading is generally not as pronounced as for $\mu$ Wave and mmWave, and hence, it is assumed that any effect of fast fading is essentially ignored in the analysis of $\mathrm{THz}$ transmissions. Second, $\mathrm{THz}$ frequencies incur high propagation loss due to severe sensitivity to rain and resonant absorption in water molecules. Accordingly, the path loss function in the case of a $\mathrm{THz}$ propagation is generally revisited by frequency-dependent molecular absorption effect with an emphasis on the LOS link as [330],

$$
\ell(r)=\left(\frac{c}{4 \pi f}\right)^{2} r^{-\alpha_{\mathrm{LOS}}} \mathrm{e}^{-k(f) r},
$$

where $k(f)$ is the medium absorption coefficient at frequency $f, \alpha_{\mathrm{LOS}}$ is the path loss exponent in the LOS region, and $c$ is the speed of light. Third, besides its effect on path loss, molecular absorption from $\mathrm{THz}$ EM rays in the medium is reemitted out of phase at the same frequencies it was initially absorbed, resulting in a source of noise dependent on operating frequencies, also known as molecular noise.

Using tools from SG, couple of works are developed in the literature for modeling and analysis of systems operating in $\mathrm{THz}$ frequencies [331]-[335]. In particular, due to the previous distinctive features of $\mathrm{THz}$ bands, deriving the Laplace functional of interference is not feasible and evaluating system performance is quite challenging. In this way, some simplifying assumptions are usually included to enhance mathematical convenience. These include treating only the effect of thermal noise, while molecular noise is assumed to be very weak and ignored. Also, instead of resorting to the Laplace functional of the interference, the authors of [331] propose to use the Campbell theorem to infer the mean of the interference in a PPP distributed THz-only network. Furthermore, Taylor expansions were adopted to approximate the mean and variance of SIR/SINR. In [332], the SIR distribution is derived for a dense Poisson THz-only network where the interference is approximated by a logistic distribution in which the parameters are fitted from the theoretical moments obtained from the Campbell's and PGFL's theorems.

In [333], coverage probability and ergodic rate are approximated for a network setup where BSs are expected to provide $\mu$ Wave or THz links to their intended UEs based on the transmission distance and the ability to establish a LOS link. In this way, when the typical UE is attached to a $\mathrm{THz} \mathrm{BS}$, interference is ignored, as well as path loss and noise generated by molecular absorption, which enable to derive performance metrics based on the PPP void probability, offering then increased computational efficiency. In [334], downlink coverage probability has been derived in a twotier cellular network composed of PPP distributed $\mu$ Wave and THz BSs such that the typical UE can select the serving BS based on the biased cell association policy. Interestingly, the lack of fading in $\mathrm{THz}$ transmissions preventing the use of the flexible Laplace transform for interference evaluation, has been tackled by judiciously using the Gil-Pelaez inversion theorem (i.e., technique \#8). The analysis provides accurate results for coverage probability but may be extremely tedious to compute given the use of several infinite sums and products.

SG is used in [335] as a powerful analytical tool to quantify the connection uncertainty when using the potential of $\mathrm{THz}$ frequencies to meet high-rate and high-reliability low latency communications requirements for wireless virtual reality users. In particular, the probability of a LOS link is first abstracted from a combination of self-blockage in which the user's body blocks a fraction of BSs due to its uniform orientation in $[0,2 \pi]$, and dynamic blockers moving in a random direction with a given velocity where the distribution of the blocked interval is obtained as the busy period distribution of a queuing system. Next, the instantaneous rate is quantified by 
TABLE IX

KEY MODELING CHOICES OF SG-BASED MODELING AND ANALYSIS OF THZ WIRELESS NETWORKS

\begin{tabular}{|c|c|c|c|c|c|c|c|c|c|c|}
\hline References & Technologies & Fading & Molecular noise & PP of nodes & Blockages model & Association policy & Analytical techniques & \begin{tabular}{|l} 
Performance Metrics \\
\end{tabular} & \begin{tabular}{|l|} 
Analytical accuracy \\
\end{tabular} & Analytical complexity \\
\hline [331] & THz-only networks & Ignored & Adopted & PPP & Decreasing exponential function & $\begin{array}{l}\text { The desired receiver is located } \\
\text { at a fixed location }\end{array}$ & $\begin{array}{l}\text { The Campbell theorem to estimate } \\
\text { the mean interference and Taylor } \\
\text { expansion to estimate mean and } \\
\text { variance of SIR/SINR }\end{array}$ & SIR/SINR mean and variance. & Approx. & Low \\
\hline [332] & Dense THz-only networks & Ignored & Ignored & PPP & Ignored & $\begin{array}{l}\text { The desired receiver is located at } \\
\text { a fixed location }\end{array}$ & $\begin{array}{l}\text { Interference is estimated by a logistic } \\
\text { distribution in which the parameters } \\
\text { are fitted from the theoretical moments } \\
\text { obtained from the Campbell's and } \\
\text { PGFL's theorems. }\end{array}$ & Success probability & Approx. & Medium \\
\hline [333] & Hybrid $\mu$ Wave/THz networks & Ignored & Ignored & PPP & Decreasing exponential function & The nearest neighbor association & Poisson void probability & \begin{tabular}{|l|l}
$\begin{array}{l}\text { SIR distribution and ergodic } \\
\text { rate }\end{array}$ \\
\end{tabular} & Approx. & Low \\
\hline [334] & Hybrid $\mu$ Wave/THz networks & Ignored & Ignored & PPP & Ignored & The Biased cell association & \begin{tabular}{|l} 
The Gil-Pelaez inversion \\
theorem (Technique \#8)
\end{tabular} & Rate distribution & Exact & Medium \\
\hline [335] & $\begin{array}{l}\text { Dense THz networks for wireless } \\
\text { virtual reality }\end{array}$ & Ignored & Adopted & MHPP & $\begin{array}{l}\text { Decreasing exponential function based } \\
\text { on a combinition of self-blockage and } \\
\text { dynamic blockers }\end{array}$ & $\begin{array}{l}\text { The desired receiver is located } \\
\text { at a fixed location }\end{array}$ & $\begin{array}{l}\text { Estimating interference by } \\
\text { a normal distribution }\end{array}$ & Mean delay & Approx. & Medium \\
\hline
\end{tabular}

approximating the interference by a normal distribution with mean and variance parameters obtained from the Campbell theorem. Finally, the mean delay is derived assuming a spatiotemporal modeling of the traffic as a function of the mean rate, the LOS probability, and the required traffic size.

Table IX outlines some of the key studies developed in the literature for the modeling and analysis of SG-based THz wireless networks, ordered in terms of their analytical accuracy and complexity.

3) Visible light communication: Using light-emitting diodes (LEDs) in license-free visible light spectrum from 400 to $800 \mathrm{THz}$ and wavelengths from 375 to $780 \mathrm{~nm}$, VLC can offer simultaneous high brightness illumination and high indoor data rate [31]. That is, information bits are modulated onto the intensity of the emitted light, where the path loss function is expressed under the form \#4 in Table V, such that the path loss exponent is mapped to the Lambertian emission order of the LED light [336, Equation 11]. Also, given the reduced VLC wavelength combined with the vicinity of receivers detection area, multipath fading is generally ignored in VLC networks.

Based on SG frameworks, we can discern three typical lines of research in the literature. i) Performance evaluation of multiuser VLC networks where the SINR statistics are evaluated under the setup of a VLC-only system. For instance, the authors of [336] evaluated the downlink performance of a VLC network under two extreme deployments of LED APs in the ceiling, namely PPP and regular lattice. The analytical framework is promising but remains intractable since the SINR distribution is expressed as a function of the Gram-Charlier series and Laguerre polynomials requiring efficient numerical computation. In [337], a novel SG framework is developed by considering a 3D model and idle mode capability at VLC APs. Coverage probability is next derived based on successive statistical equivalences of SINR, but the approach requires fundamental revisions in the way to address the lack of a fading term in VLC networks. ii) Optimizing hybrid VLC/RF networks such that a joint operation of both technologies is evaluated. For instance, the authors of [338] considered a setup of several configurations of coexisting RF/VLC networks to derive coverage probability based on techniques \#5 and \#8 in Tab VI. In [339], the outage probability is first derived in a VLC/RF system by approximating the interference as a sum of gamma distributions (technique \#10 in Tab VI), and an optimization problem is next formulated to optimize the density of VLC/RF nodes enabling higher EE under an outage probability constraint. Furthermore, due to the inherent broadcast nature of VLC networks, data transfer may be subject to fraudulent eavesdropping. Several papers have considered iii) secrecy enhancement in VLC networks, where the physical layer (PHY) is exploited to prevent the information-theoretic security from interception [340]-[342].

4) Free-space optical communications: Using signals with wavelength range in 785-1550 nm, free-space optical (FSO) communication is a laser beam for high data rate transmissions in a point-to-point free space setup, where it can serve as a promising backhaul solution to avoid expensive or not feasible deployments of wired connections [42], [343].

Despite the potential benefits of FSO communications, SG as a powerful analytical tool has not been sufficiently used in the evaluation of FSO networks due to several major modeling challenges. To the authors' knowledge, it is only recently that the first PPP abstraction model has been leveraged in performance evaluation of FSO networks [344], where a scenario of SWIPT through laser beams emitted from the ground to UAV-mounted BSs is considered. In fact, the use of SG in modeling FSO communications is generally challenging due to the following aspects: i) FSO narrow beams require a perfect alignment of the LOS path, which can be problematic due to building sway generated by some environmental factors. Such feature needs to be captured by a random process to be introduced into performance analysis [42]. ii) Urban FSO is very sensitive to weather conditions ${ }^{13}$, which can be typically captured by an attenuation function dependent on distance, rain, and snowfall rate [345], [346]. iii) The FSO signal is also attenuated by atmosphere molecular absorption dependent on the wavelength of the transmitted signal. iv) Such signal can also be constrained by fluctuations in temperature and humidity gradients over time, also known as scintillation or turbulence-induced fading. The universal model to capture such turbulence conditions is to consider doubly stochastic fading models [42], [343], [347]. Last but not least v) the FSO link is also subject to undesirable ambient noise caused by photons radiations of sunlight [348].

\footnotetext{
${ }^{13}$ Several techniques are considered to overcome such impairements, for example using a mmWave backup link to supplement the FSO main link during adverse weather conditions [345], or using relay-assisted transmissions where the overall FSO path is splitted into small paths with reduced losses [346].
} 


\section{B. Stochastic Geometry for Cognitive Wireless Networks}

Cognitive radio (CR) is a promising technology to address the scarcity of the licensed spectrum. CR techniques ensure actually an opportunistic allocation of the available spectrum where secondary users, also known as cognitive users, can scan and access the unused spectrum portions at specific time or place without impairing existing primary users [349]. The literature is rich in contributions dealing with the use of SG to evaluate the benefits of various spectrum sharing schemes. The fundamental challenge actually is how to use SG tools to capture the availability of unused licensed spectrum portions. One key approach is to consider geographical regions where cognitive users are less likely to impair the performance of primary users.

The analysis of the literature shows that there are generally three generative ways to capture such event: i) The guard zone approach [105], [350], in which the secondary user is allowed to transmit as long as it is outside an exclusion region around primary users. The locations of active cognitive users can be modeled for example by a PHP [105], or a MHP [350]. Such coupling in the locations of active cognitive and primary users via exclusion regions renders the analysis of interference very challenging as no tractable expression of the PGFL is available, and then only some estimates of the aggregate interference are obtained. ii) The max-received power approach [351], in which the process of active cognitive users is derived as an independent thinning based on the probability that the maximum instantaneous signal power at the level of a random secondary user and sent by active primary users is below a certain threshold. iii) The outage probability approach [352], in which the location of active cognitive users follow a PPP with a specific density in such a way to guarantee that the induced outage probability at the level of the primary network will not exceed a predefined threshold.

Furthermore, CR capability can be used beyond the conventional primary/secondary users setup, typically as a promising technique for distributed interference mitigation in co-channel deployments of HetNets [353]. That is, Femto BSs equipped with $\mathrm{CR}$ abilities, can sense the spectrum usage in intra-tier and cross-tier layers and hence select the appropriate spectrum sharing policy to avoid severe interference.

\section{Multiple-Input Multiple-Output Systems}

In conjunction with adding new cells and using higher frequency bands, MIMO technology is considered as a key component in the race towards higher data rates in 5G/B5G networks [268]-[270]. MIMO is usually used to increase spatial diversity and combat channel fading, which enhances the reliability of the reception. Alternatively, fading can be seen as a source of increasing the degrees of freedom in MIMO systems. That is, the receiving antenna array can retrieve independent information streams with sufficiently different spatial signatures, which helps improve data rate. This technique is referred to as spatial multiplexing. The third popular use case of MIMO is precoding or multi-flow beamforming, wherein the same information symbol is sent by each of the transmitting antennas with appropriate phase and gain weighting, so that the signal power is maximized at the receiver by constructively adding signals emitted by different antennas. Accordingly, channel estimation and symbol detection is a key challenge in MIMO systems.

Most research works evaluating the performance of MIMO systems has considered the one cell scenario where interference from other cells is neglected. The performance analysis of a multi-cell network is however much more challenging due to geometrical properties between cells and the resulting intercell interference. In such a context, SG provides a set of powerful tools for performance modeling and analysis of several MIMO techniques. A first premise that worth be considered in SGbased models for MIMO systems is that a mapping between the number of user antennas and the number of BS antennas under Rayleigh fading channels, i.e. exponentially distributed channel power gains, can tractably define the implemented MIMO technique. It is then possible to formulate the SINR within a SISO setup in such a way that the channel power gain for the desired and interference signals are gamma distributed with a scale of 1 and a shape defined as a function of the number of user's and BS's antennas [354, Table I] [355, Table II]. In this way, the gamma distribution is widely adopted in the performance analysis of SG-based MIMO systems, where the key task is to compute the $n$-th derivatives of the Laplace transform, which resorts to the approximations discussed in technique \#5 of Table V.

MIMO can be leveraged as a multi-user system (MUMIMO) where several antennas at the BS can serve simultaneously a number of users. To decode the data transmitted simultaneously by multiple users, each BS needs the channel knowledge of its associated users, which is estimated by a set of orthogonal uplink pilot sequences, while channel reciprocity is exploited at the BS to pre-encode data in the downlink. Due to the limited size of the coherence block and also the limited number of orthogonal pilot sequences available for channel estimation, pilot sequences are reused in different cells, giving rise to coherent interference between UEs sharing the same pilots, also known as pilot contamination (PC).

There are mainly three key approaches used in the SGbased literature to address the issue of PC. i) The regular pilot (RP) transmission approach in which the transmission of pilot and data symbols is done separately in the coherence block to minimize interference in the channel estimation process [356]. ii) The superimposed pilot (SP) approach consisting of simultaneous transmission of pilot and data signals [357]. SP allows the use of longer pilot sequences, as compared to RP, which reduces pilot contamination at the expense of increased estimation overhead, which in turn, reduces the amount of data symbols transmitted per coherence block. Also, simultaneous transmission of pilot and data signals in SP will introduce interference into the channel estimation process from data symbols. The previous approaches consider the use of fully orthogonal pilots between cells, while the spectral efficiency can be further improved by using a more dynamic pilot reuse scheme. iii) The third approach thus considers fractional pilot reuse (FPR), where the users of one cell are split into two groups, i.e. center of cell (CC) and edge of cell (EC) users 
[358]. While all pilots for CC users are reused in each cell, pilots for EC users are reused in specific cells according to the reuse factor. For tractability, $\mathrm{CC}$ region is captured by the Johnson-Mehl cell of its BS while the remainder of the Poisson Voronoi cell is assumed to be the EC region.

\section{The Promising Approach of Metasurfaces}

Despite relatively lower costs and easy deployment of RNs as compared to macro and small cells, extensive deployment of RNs in UDNs can increase costs in view of their inherent power consumption and OPEX costs. Recently, a radically new wireless communication paradigm has been proposed [32], [33], [359], wherein some software-controlled metallic reflectors made of low-cost passive elements, i.e., metasurfaces, are judiciously placed in environmental objects and/or aerial platforms to support communication of edge terrestrial and/or aerial users [360]. As elaborated in [359], an RIS is usually made of tiny scattering elements that are controlled by low-cost and low-power electronic circuits that enable their configurability over time. In general, an RIS requires many tunable elements in order to get similar performance as other transmission technologies, e.g., relays [361]. Broadly speaking, RISs make a tradeoff between the low complexity of their electronic circuits, the absence of power amplifiers and multiple RF chains with a large number of the available scattering elements.

Quantifying the performance of RIS-assisted wireless networks, especially in large-scale deployments, require new analytical tools along two main directions: i) the development of link-level models for RISs that allow us to quantify the power scattered by an RIS as a function of its configuration; and ii) the amalgamation of the resulting link-level models with SG in order to quantify network-level performance metrics [362], [363].

As far as the analysis of the power scattered by an RIS is concerned, this is an open research issue and some attempts have recently been made [364]-[373]. In [365], in particular, it is shown that the electromagnetic field scattered by an RIS depends on several factors, which include the electrical size of the RIS and transmission distances between the transmitter, the RIS, and the receiver. In general, the scattered electromagnetic field is formulated in terms of an integral (or a finite sum), which can be formulated in a closed-form expression only in some asymptotic regimes, e.g., when the size of the RIS is very large or is very small. In these operating regimes, the scaling laws of the propagation distances may be different. The surface gain offered by an RIS also depends on the specific function that it needs to realize. The study conducted in [365] is applicable to homogenized (or virtually continuous) RISs. In [369], the authors have recently introduced a communication model for discrete-type RISs, which resembles a MIMO communication link. The communication model in [369] has recently been used in [373] for system optimization. A major limitation of the available link-level models to quantify the electromagnetic field scattered by an RIS is that they usually apply to free-space channels.

As far as the amalgamation of link-level models for RISs with SG is concerned, the resulting problem is non-trivial and open [362], [363]. This is due to two main reasons. i) Existing link-level models for RISs are applicable to free-space channels and are, in general, formulated in terms of integrals or summations. This makes their integration in conventional SG frameworks difficult. The analytical frameworks available for some asymptotic regimes, e.g., in [365], are only in part applicable in a SG framework since they are accurate only for some specific transmission distances. Since in SG-based analysis the transmission distance is a random variable and the users may be very close to the RIS, accurate path-loss models need to be used. This implies that, for application to SG-based studies, link-level models for RISs need to account for the near-field regime. This is briefly elaborated in [365]. ii) RISs are expected to be deployed on environmental objects, such as the walls of buildings. In typical SG-based analysis, the environmental objects are usually modeled as blocking objects, while the impact of reflections and how they affect the SINR is usually ignored due to the associated analytical complexity [362], [363]. Likewise, the large size of RISs may not be compatible with the typical assumption that the links scattered by the same RISs are statistically independent of each other. This requires the development of new analytical methods for modeling RIS-coated objects in large-scale wireless networks.

Overall, the modeling, analysis, and optimization of largescale RIS-assisted wireless networks is a major open research issue, which requires the development of new tools for the link-level modeling of RIS, which needs to account for the scattering (e.g., reflections) introduced by RIS-coated objects, and which needs to consider appropriate association strategies between the users and the RIS so as to best use the available RISs [374], [375]. Finally, the development of tools that combine ML and SG for optimizing large-scale networks is another major research venue [38], [39].

\section{E. Stochastic Geometry for NOMA Networks}

Compared to conventional orthogonal multiple access techniques where UEs are served in orthogonal resource blocks, NOMA is introduced as an emerging technology enabling multiple UEs to share the same time-frequency resource block [376]. One key variant is actually power-domain NOMA [52], in which multiple UEs can use the same resource block but at different power levels. That is, UEs are first ordered according to a measure of link quality. Next, the BS superposes the UEs in the same resource block by allocating a fraction of the BS transmit power to each UE so that the worst UE in terms of link quality is assigned the highest power coefficient. In downlink ${ }^{14}$ NOMA reception, successive interference cancellation (SIC) is implemented at each UE in such a way that signals of weaker UEs are decoded and canceled from the observation, while signals of stronger UEs are treated as noise.

Based on such key components of power-domain NOMA, several research works have leveraged SG for performance analysis of NOMA wireless networks. The key modeling choices are: i) how to capture the cluster of UEs to be

\footnotetext{
${ }^{14}$ In uplink NOMA, SIC is implemented at the level of the serving BS, where signals from strong UEs are decoded and cancelled successively, while signals from weak UEs are considered as noise [52], [377].
} 
simultaneously served in the same resource block. Several setups are considered in the literature, for example, consider only the case of two UEs, typically two random UEs in the Voronoi tessellation of the serving BS, i.e., random pairing [378], [379], [381], [384], or selective pairing of a cell-center and a cell-edge UE [378]-[380], [385]. Some works assume a more general setup where the cluster is a constant number of UEs $(>2$ ) [387], [388], a bounded random number of UEs modeled as PPP [382] or as PCP [377], [386], or even two layers of user group in a NOMA-based multicast setup [383]. ii) Which measure of link quality to use in served UEs ordering. Due to its tractability, the common metric is to classify UEs based on their distance to the serving transmitter [377], [378], [382], [387], [388]. Other metrics is to consider the fading gain [379], [385], the instantaneous signal power based on fading and the path loss function [386], or the instantaneous signal power normalized by noise and inter-cell interference power [386]. Also, iii) how to introduce the effect of SIC in the SINR formulation. The generative approach is to introduce a fraction parameter $(\in[0,1])$ that reflects the accuracy of SIC [386].

\section{F. Stochastic Geometry for IBFD Technology}

IBFD wireless nodes can transmit and receive data simultaneously at the same time/frequency channel. Such capability is expected to double the spectrum efficiency at the expense of increased residual self-interference (SI) between uplink and downlink [53]. SG has been extensively used in the literature to quantify the performance gains achieved by IBFD capability [389]-[396]. The key model change is actually to account for the SI power after performing cancellation, which can be perfect, imperfect, or without prior knowledge of its effect.

Several models have been considered in the literature to capture such residual SI power gain. The common practice is to consider a constant value dependent on the transmit power, which is a typical scenario in digital cancellation techniques, where the SI intensity after cancellation can be estimated [389]-[391], [396]. However, in the context of other cancellation techniques where an estimation of the risidual SI is not feasible, e.g., analog-domain or propagation-domain schemes as pointed out in [389], the residual SI channel is generally modeled by a random variable, e.g., Rician fading [394], [395], Nakagami- $m$ fading [393]. For instance, modeling and analysis of a wireless network with random combination of HD and IBFD nodes is studied in [390], where it has been shown an enhanced success probability in HDonly networks, even under perfect SI cancellation. However, IBFD-only networks, can outperform their HD peers in terms of throughput due to higher resource utilization.

It is worth noting that the benefits of dense HetNets in terms of capacity improvements are generally limited by the spectrum scarcity. Typically, IBFD as a frequency reuse technique has been investigated in HetNets setup, where it has been reported that network throughput can be maximized under HD-only or IBFD-only HetNets rather than using a combination of them [389]. The problem of optimizing user association policy in IBFD HetNets with DUDA scheme is evaluated in [393]. Also, due to less viable wired backhaul for small cells, IBFD is investigated in [391], [395] as a promising solution for wireless backhaul of small cells. Last but not least, the potential of MIMO antennas to mitigate the extra interference introduced by IBFD is analyzed in [394], [396], while the impact of equipping MIMO RNs with IBFD capability in a cellular network with MIMO BSs is quantified in [392]. A common result is that BSs and IBFD RNs need to be equipped with sufficiently large number of antennas to achieve the expected benefits of IBFD capability.

\section{G. Stochastic Geometry for Physical Layer Security}

The usual bit-level cryptographic protocols, requiring heavy overheads and intense coordination, can be generally compromised if eavesdroppers are equipped with convenient computing capabilities. An alternative promising approach is to consider physical layer security besides the conventional error correction mechanisms in such a way to impair the channel capacity of eavesdroppers with limited impact on the QoS of legitimate users [54]. In fact, based on the Wyner's encoding scheme, a transmitter selects two rates, namely, the rate of codewords $R_{t}$ and the rate of confidential messages $R_{s}$, i.e., the secrecy rate. Reliable connection is actually achieved when the instantaneous capacity at the intended receivers is greater than $R_{t}$, while a secrecy failure event occurs when the instantaneous capacity at eavesdroppers is above $R_{t}-R_{s}$ [397].

SG is typically harnessed to evaluate the impact of key system parameters on the physical layer security of largescale wireless networks. That is, the interplay between cell association policy and the secrecy capability is investigated in [398]-[400]. Physical layer security in the context of largescale networks with NOMA is studied in [401], with MIMO in [402], and with D2D communications in [403]. The sensitivity of mmWave communications to blockages is explored in [404] to establish a tradeoff between higher data rates and enhanced secrecy. The question of secure communications in the context of a multiuser VLC network deployed in public areas under a broadcast topology is investigated in [340]-[342]. There are generally four popular techniques considered in the literature to enhance physical layer security. For instance, i) the artificial noise approach in which some artificial noise is added to secret messages in order to make decoding harder to eavesdroppers [402], [405]. ii) The secrecy guard zone approach in which confidential messages are transmitted only if eavesdroppers are outside an exclusion region around legitimate nodes [341], [397], [401]. iii) The friendly interference approach in which a friendly interference is generated to jam the channel capacity of eavesdroppers with controlled impact on the QoS of legitimate users, e.g., exploit the generated interference by D2D communications [403] or by a set of friendly jammers [406], [407]. iv) The selective transmission approach where confidential messages are transmitted selectively to users in such a way to reduce the likelihood of being intercepted by eavesdroppers, e.g., using directional antennas [397], or the transmit antenna selection technique [408]. 


\section{TOWARDS NEW HORIZONS FOR STOCHASTIC GEOMETRY}

After intensive use of SG in modeling and analysis of communication networks, notably during the last decade of the seminal results [16]-[18], the research community begins to experience some congestion on applications of SG and some degree of duplication in the literature. This is in particular due to the following reasons: i) SG is very rich in theory but only few results are used practically in modeling and analysis of wireless networks, including Campbell's and PGFL theorems, as well as constructing properties that may preserve the Poisson law (e.g., superposition, displacement, mapping, independent thinning). ii) Given some modeling and design challenges, SG has not been sufficiently explored in the analysis of promising research areas (e.g., networks with metasurfaces, molecular communication (MC), ML, FSO for backhaul or satellites communications). iii) No bridges of interaction are created between SG and other emerging mathematical theories, which can give rise to new practical results of SG. In this section, we will outline some emerging research avenues that can revitalize the use of SG during this decade.

\section{A. Stochastic Geometry for Modeling and Analysis of Molec- ular Communications}

One promising frontier of conventional EM communication systems is the ability to share, manipulate and control information on a very small scale in such a way to connect swarms of intelligent autonomous nano-devices, i.e., devices in a scale ranging from 1 to 100 nanometers, e.g., nano-robots, nanoprocessors, nano-clocks. Based on biological communication in nature where molecules are the basic carriers of information, $\mathrm{MC}$ is expected to be one of the next big ${ }^{15}$ ideas of communication due to its inherent biocompatibility and enhanced EE at the cost of slow propagation speed as compared to EM wave based communications [409], [410].

The vision of molecular nanonetworks is actually fraught with many challenges, among them, how information can be encoded in molecules and how such molecules are supposed to propagate from a transmitter to a receiver. Several MC propagation schemes are considered in the literature such as diffusion-based propagation, flow assisted propagation and bacteria chemotaxis. The most common approach is the free diffusion of particles where molecules can propagate from one point to another in a random Brownian motion via inherent thermal energy, which does not require any external source of energy and may induce confusion in molecules detection at the receiver, also known as inter-symbol-interference. Several ways are considered to encode information in such diffusing particles, for example encoding information according to the time of arrival of molecules at the receiver, according to molecular composition or to the variations on molecules concentration in the space.

\footnotetext{
${ }^{15}$ As was first pointed out conceptually by the 1965 nobel laureate physicist Richard Feynman in his famous speech entitled "There's Plenty of Room at the Bottom" in Dec. 1959.
}

Another major constraint in $\mathrm{MC}$ systems is the laborious and expensive nature of laboratory experimentation which justifies the wide use of simulation environments for MC analysis [411]. Interestingly, the authors of [412] presented a first attempt to provide some appropriate analytical tools via $\mathrm{SG}$ in such context of miniaturization in MC. The work proposed a mathematical framework for performance evaluation of a 3D diffusion-based large-scale MC system. The average number of sensed particles and the bit error probability at a receiver located at the origin are next characterized over many spatial realizations of a swarm of point transmitters scattered in space according to some PPP and emitting the same bit sequence (the same type of molecule) simultaneously, i.e., cochannel transmitters. Analytical evaluation of $\mathrm{MC}$ as a serious alternative to EM wave based systems, particularly in strong attenuation regimes of EM waves, is relatively new and several fundamental questions need actually years and years to be answered and agreed about [413]. However, many advances are expected in the near future due especially to recent development in inexpensive testbed for MC systems capable of transmitting short text messages via chemical signals [411].

\section{B. Stochastic Geometry in the Era of Machine Learning}

SG and ML have recently been considered as the most popular methods with renewed and widespread interest in the design and analysis of wireless networks. The former is actually a powerful model-driven approach aimed to enhance the tractability and accuracy of conventional probabilistic models, e.g., channel, interference, scheduling, by considering the randomness on the locations of the transmitters and/or the receivers, so that one can evaluate performance metrics of wireless networks upon several realizations of network geometry [2], [18]. The latter is however, a data-driven simulationbased approach, which by collecting sufficient amount of realistic data, i.e., the training set, can feed a supervised and/or unsupervised learning process deployed at the cloud components of the network, to enable the prediction of the desired result, e.g., performance metrics [414], [415].

To the best of the authors' knowledge, there are fundamentally two lines of thought in the literature regarding the mode of interaction that should prevail between ML and SG. The first vision is based on an evolutionary interaction [414][416], in which ML is conceived as a separate evolved alternative to SG enabling to overcome the shortcomings of the latter and provide more accurate representation of reality. In fact, SG model-driven approach is generally governed by a tradeoff between tractability and accuracy, where tractable models are simply less accurate to reflect realistic scenarios, while precise models are hard to derive and their resulting algorithms are too complex to implement. However, with the unprecedented availability of data, inducing the need for software-controlled and optimized operations, in addition to recent developments in smart radio environments via the use of metasurfaces [32], [362], it is difficult to develop accurate SG models that can capture such complex scenarios of analysis due to the unlimited degrees of freedom and system constraints. The second vision is based on a collaborative interaction 
[38]-[41]; in which a common ground and potential crossfertilization between SG and ML is created such that the strength of both approaches will be jointly harnessed to tackle the same issue. For instance, SG models can be integrated as a hypothesis class in the learning process of ML. One representative example is the class of problems known as the subset selection problems, where an optimal subset needs to be selected from a ground set. In such context, SG probabilistic models constructed by finite DPPs are used to feed ML datadriven supervised learning frameworks.

\section{Stochastic Geometry as a Multi-objective Analytical Tool}

From the previous discussions, it is evident that future 5G/B5G wireless networks are going to be highly heterogeneous, multi-layered, with embedded intelligence at both the core and the edge of the network, where ML is expected to play a crucial role in link and system-level decisions. In such a context, future performance metrics need to be carefully tailored to ensure the joint evaluation of throughput, latency, and reliability, which ultimately leads to the joint optimization of communication, control, localization, sensing, energy consumption, and many other parameters and resources. Interestingly, SG can be envisioned as a potential analytical candidate in this way.

For instance, Fig. 10 presents some scenarios where the SINR can be combined with useful utility functions to enable the joint analysis of communication and other related attributes, e.g., DUDA, SWIPT [417], [418]. Also, SG is increasingly adopted in studying the joint localization and communication of users in a given propagation environment. In particular, the authors of [419], [420] use tools from SG and its inherent PP theory to evaluate the statistics of the number of BSs/anchors (i.e., nodes with known positions) that can participate in the localization procedure of users/agents (i.e., nodes with unknown positions) as a function of systemlevel parameters and channel impairments. Typically, there is a tradeoff, known as the hearability problem, that needs to be considered between communication requirements that ask for a strong signal from the desired BS and a poor one from interferers, versus localization that requires a good signal from most BSs.

Furthermore, SG and its inherent random set theory are becoming widely adopted for the analysis of combined recognition, data manipulation, and movement in real-world environments. Typically, SG is used to study uncertainty in geometric objects in order to build models from IoT measurements [421], [422]. The physical environment landmarks are actually abstracted into parametric representations such as points, lines, and edges. These features are next handled as realizations of random variables modeled as a finite random set, which based on the Bayesian estimation paradigm, can allow to jointly estimate the number of objects and their states. This is relevant for example to detect and locate objects from surveillance images [421]. Besides, random set theory is gaining increased importance for providing a theoretical estimation for the famous simultaneous localization and map building (SLAM) problem that asks for the ability to place an autonomous robot at an undefined location in an undefined environment and construct a map based only on relative ambient observations, and subsequently use this map for spatial mobility of this robot [422]. Using random finite set theory, SLAM is then presented as a Bayesian filtering problem in which the joint recursive estimation of the robot route and set-valued map are spatially distributed over time as measurements are acquired.

Future research efforts are therefore expected to identify attractive applications of SG in multi-objective optimization.

\section{Grothendieck Toposes as Mathematical Bridges for Stochastic Geometry}

One effective way to deal with the embarrassing tradeoff between tractability and accuracy that governs SG models, is to investigate how to build more advanced and accurate SG models from tractable and easy-to-interpret models conceived by other mathematical fields. Our vision therefore is to create some abstract bridges of interaction between SG and other mathematical fields where we have reached some remarkable degree of specialization and proficiency. The rationale is to create meaningful and powerful analogies that may illuminate concepts and suggest new practical results in SG. A promising approach to meet such aims is through the concept of topos introduced by Alexandre Grothendieck during his Seminar on Algebraic Geometry in the early sixties.

"It is the notion of topos that is this "bed" where come to be married geometry and algebra, topology and arithmetic, mathematical logic and category theory, the world of the continuous and that of discontinuous or discrete structures. It is the most vast thing I have conceived, to grasp with finesse, through the same language rich in geometric resonances, an "essence" common to situations most distant from each other, coming from one region or another of the vast universe of mathematical things."-Alexandre Grothendieck commented in his famous text of autobiographical reflections "Récoltes et Semailles" [423].

Recently, new perspectives on the notion of topos have emerged. According to Olivia Caramello [424], Grothendieck toposes can be used as unifying spaces that can serve as bridges for transferring properties, ideas, and results between distinct mathematical theories. In our case, between SG and other mathematical fields, so that long-standing problems formulated in SG can be solved using techniques from a different field, and results in a well known area can be appropriately transferred to results in SG.

\section{CONCLUSION}

In this paper, we surveyed and investigated PP models, statistical tools, SG system model preferences, and the major analytical techniques extracted from the rich legacy of SGbased research works, conducted over the past decade in modeling and analysis of wireless networks. We also outlined how SG has been considered to capture the properties of new RANs and quantified the benefits of a number of $5 \mathrm{G} / \mathrm{B} 5 \mathrm{G}$ enabling technologies. The main goal is to review the milestones established in the past decade in the usage of SG for wireless 
networks and to predict the challenges in the upcoming decade in the light of $5 \mathrm{G} / \mathrm{B} 5 \mathrm{G}$ emerging paradigms.

The insights presented in this paper illustrate the flexibility of SG and its ability to capture the analysis of the rather unconventional scenarios; these features of SG will likely enable it to remain as an essential tool in modeling and analysis of future wireless networks, especially given its potential of cross-fertilization with ML and its expected role in the analysis of emerging wireless communication systems and network architectures. Also, the Grothendick's toposes is a powerful mathematical concept that can illuminate novel seminal results into $\mathrm{SG}$ and hence pave the way for the next generation use cases of SG in wireless networks.

\section{REFERENCES}

[1] D. Stoyan, W. Kendall, and J. Mecke, Stochastic Geometry and Its Applications, 2nd Edition, 2nd ed. John Wiley and Sons, 1996.

[2] F. Baccelli, M. Klein, M. Lebourges, and S. Zuyev, "Stochastic geometry and architecture of communication networks," J. Telecommunication Systems, vol. 7, no. 1, pp. 209-227, Jun. 1997.

[3] F. Baccelli and S. Zuyev, "Stochastic geometry models of mobile communication networks," Frontiers in Queueing, CRC Press, pp. 227243, 1997.

[4] F. Baccelli and B. Blaszczyszyn, Stochastic Geometry and Wireless Networks in Foundations and Trends in Networking, vol. 1, Now Publishers, 2009.

[5] F. Baccelli and B. Blaszczyszyn, Stochastic Geometry and Wireless Networks in Foundations and Trends in Networking, vol. 2, Now Publishers, 2009.

[6] M. Haenggi, Stochastic Geometry for Wireless Networks. Cambridge, U.K.: Cambridge Univ. Press, 2012.

[7] D. J. Daley and D. Vere-Jones, An Introduction to the Theory of Point Processes, Elementary Theory and Methods, vol. 1, Springer-Verlag New York, 2003.

[8] J. F. Coeurjolly, J. Møller, and R. Waagepetersen, "A tutorial on Palm distributions for spatial point processes," Int. Stat. Review, vol. 85(3), pp. 404-420, Dec. 2017.

[9] R. Schneider and W. Weil, Stochastic and integral geometry, Probability and its Applications (New York), Springer-Verlag, Berlin, 2008.

[10] L. A. Santaló, Integral Geometry and Geometric Probability. Cambridge, U.K.: Cambridge Univ. Press, 1976.

[11] G. Matheron, Random Sets and Integral Geometry. New York, NY: John Wiley and Sons, 1975.

[12] J. Grandell, "Point processes and random measures," Advances in Applied Probability 9, pp. 502-526, 1977.

[13] D. Coupier, Stochastic Geometry: Modern Research Frontiers. Cham, Switzerland : Springer, 2019.

[14] J. Illian, A. Penttinen, and H. Stoyan, Statistical Analysis and Modelling of Spatial Point Patterns. Statistics in Practice, Wiley-Blackwell, 2008.

[15] E. N. Gilbert, "Random plane networks," SIAM J., vol. 9, pp. 533-543, 1961.

[16] M. Haenggi, "A geometric interpretation of fading in wireless networks: theory and applications," IEEE Trans. Inf. Theory, vol. 54, no. 12, pp. 5500-5510, Dec. 2008.

[17] F. Baccelli, B. Blaszczyszyn, and P. Muhlethaler, "Stochastic analysis of spatial and opportunistic aloha," IEEE J. Sel. Areas Commun., vol. 27, no. 7, pp. 1105-1119, Sep. 2009.

[18] J. G. Andrews, F. Baccelli, and R. K. Ganti, "A tractable approach to coverage and rate in cellular networks," IEEE Trans. Commun., vol. 59, no. 11, pp. 3122-3134, Nov. 2011.

[19] S. Srinivasa and M. Haenggi, "Distance distributions in finite uniformly random networks: theory and applications," IEEE Trans. Veh. Technol., vol. 59, no. 2, pp. 940-949, Feb. 2010.

[20] H. S. Dhillon, R. K. Ganti, F. Baccelli, and J. G. Andrews, "Modeling and analysis of K-Tier downlink heterogeneous cellular networks," IEEE J. Sel. Areas Commun., vol. 30, no. 3, pp. 550-560, Apr. 2012.

[21] H.-S. Jo, Y. J. Sang, P. Xia, and J. G. Andrews, "Heterogeneous cellular networks with flexible cell association: A comprehensive downlink SINR analysis," IEEE Trans. Wireless Commun., vol. 11, no. 10, pp. 3484-3495, Oct. 2012.
[22] T. D. Novlan, H. S. Dhillon, and J. G. Andrews, "Analytical modeling of uplink cellular networks," IEEE Trans. Wireless Commun., vol. 12, no. 6, pp. 2669-2679, Jun. 2013.

[23] S. Zuyev, "Stochastic geometry and telecommunications networks," in Stochastic Geometry: Highlights, Interactions and New Perspectives, W. S. Kendall and I. Molchanov, Eds. Oxford University Press, 2009.

[24] M. Haenggi, J. G. Andrews, F. Baccelli, O. Dousse, and M. Franceschetti, "Stochastic geometry and random graphs for the analysis and design of wireless networks," IEEE J. Sel. Areas Commun., vol. 27, no. 7, pp. 1029-1046, Sep. 2009.

[25] H. ElSawy, E. Hossain, and M. Haenggi, "Stochastic geometry for modeling, analysis, and design of multi-tier and cognitive cellular wireless networks: A survey," IEEE Commun. Surveys Tuts., vol. 15, no. 3, pp. 996-1019, Thirdquarter 2013.

[26] J. G. Andrews, A. K. Gupta, and H. S. Dhillon, "A primer on cellular network analysis using stochastic Geometry," arXiv preprint, Oct. 2016 , [Online]. Available: https://arxiv.org/abs/1604.03183.

[27] I. Chih-Lin, S. Han, Z. Xu, Q. Sun, and Z. Pan, "5G: Rethink mobile communications for 2020+," Philosoph. Trans. Roy. Soc. A Math. Phys. Eng. Sci., vol. 374, no. 2062, 2016, Art. no. 20140432.

[28] 6G Flagship, University of Oulu, September 2019, "Key drivers and research challenges for $6 G$ ubiquitous wireless intelligence" [Online]. Available: http://jultika.oulu.fi/files/isbn9789526223544.pdf.

[29] Z. Pi and F. Khan, "An introduction to millimeter-wave mobile broadband systems," IEEE Commun. Mag., vol. 49, no. 6, pp. 101-107, Jun. 2011.

[30] H. Song and T. Nagatsuma, "Present and future of teraHertz communications," IEEE Trans. Terahertz Science and Technol., vol. 1, no. 1, pp. 256-263, Sep. 2011

[31] P. H. Pathak, X. Feng, P. Hu, and P. Mohapatra, "Visible light communication, networking, and sensing: A survey, potential and challenges," IEEE Commun. Surveys Tuts., vol. 17, no. 4, pp. 20472077, Fourthquarter 2015.

[32] C. Liaskos, S. Nie, A. Tsioliaridou, A. Pitsillides, S. Ioannidis, and I. Akyildiz, "A new wireless communication paradigm through softwarecontrolled metasurfaces," IEEE Commun. Mag., vol. 56, no. 9, pp. 162-169, Sep. 2018.

[33] M. Di Renzo et al., "Smart radio environments empowered by reconfigurable AI meta-surfaces: an idea whose time has come," EURASIP J. Wireless Commun. Netw., vol. 129, no. 1, pp. 1-20, May 2019.

[34] M. Mozaffari, W. Saad, M. Bennis, Y. Nam, and M. Debbah, "A Tutorial on UAVs for wireless networks: Applications, challenges, and open problems," IEEE Commun. Surveys Tuts, vol. 21, no. 3, pp. 23342360, Thirdquarter 2019.

[35] L. Li, G. Zhao, and R. S. Blum, "A survey of caching techniques in cellular networks: research issues and challenges in content placement and delivery strategies," IEEE Commun. Surveys Tuts., vol. 20, no. 3, pp. 1710-1732, Thirdquarter 2018.

[36] A. K. Gupta, X. Zhang, and J. G. Andrews, "SINR and throughput scaling in ultradense urban cellular networks," IEEE Wireless Commun. Lett., vol. 4, no. 6, pp. 605-608, Dec. 2015.

[37] A. AlAmmouri, J. G. Andrews, and F. Baccelli, "A unified asymptotic analysis of area spectral efficiency in ultradense cellular networks," IEEE Trans. Inf. Theory, vol. 65, no. 2, pp. 1236-1248, Feb. 2019.

[38] A. Zappone, M. Di Renzo, M. Debbah, "Wireless networks design in the era of deep learning: model-based, AI-based, or both?," IEEE Trans. Commun., vol. 67, no. 10, pp. 7331-7376, Oct. 2019.

[39] A. Zappone, M. Di Renzo, M. Debbah, T. T. Lam, and X. Qian, "Model-aided wireless artificial intelligence: embedding expert knowledge in deep neural networks for wireless system optimization," IEEE Veh. Technol. Mag., vol. 14, no. 3, pp. 60-69, Sep. 2019.

[40] B. Blaszczyszyn and H.P. Keeler, "Determinantal thinning of point processes with network learning applications", in Proc. IEEE Wireless Commun. Netw. Conf. (WCNC), Marrakech, Morocco, Apr. 2019.

[41] C. Saha, H. S. Dhillon, "Machine learning meets stochastic geometry: determinantal subset selection for wireless networks", in Proc. IEEE Glob. Commun. Conf. (GLOBECOM), Waikoloa, HI, Dec. 2019.

[42] D. Kedar and S. Arnon, "Urban optical wireless communication networks: the main challenges and possible solutions," IEEE Commun. Mag., vol. 42, no. 5, pp. S2-S7, May 2004.

[43] J. Wildman, P. H. J. Nardelli, M. Latva-aho, and S. Weber, "On the joint impact of beamwidth and orientation error on throughput in directional wireless Poisson networks," IEEE Trans. Wireless Commun., vol. 13, no. 12, pp. 7072-7085, Dec. 2014.

[44] J. G. Andrews, "Seven ways that HetNets are a cellular paradigm shift," IEEE Commun. Mag., vol. 51, no. 3, pp. 136-144, Mar. 2013. 
[45] J. G. Andrews, R. K. Ganti, M. Haenggi, N. Jindal, and S. Weber, "A primer on spatial modeling and analysis in wireless networks," IEEE Commun. Mag., vol. 48, no. 11, pp. 156-163, Nov. 2010.

[46] D. Moltchanov, "Distance distributions in random networks," Ad Hoc Netw., vol. 10, pp. 1146-1166, Aug. 2012.

[47] M. Haenggi and R. Ganti, Interference in Large Wireless Networks, in Foundations and Trends in Networking, NOW Publishers, vol. 3, no. 2, pp. 127-248, 2008.

[48] P. Cardieri, "Modeling interference in wireless ad hoc networks," IEEE Commun. Surveys Tuts., vol. 12, no. 4, pp. 551-572, Fourthquarter 2010.

[49] H. ElSawy, A. Sultan-Salem, M.-S. Alouini, and M. Z. Win, "Modeling and analysis of cellular networks using stochastic geometry: A tutorial," IEEE Commun. Surveys Tuts, vol. 19, no. 1, pp. 167-203, Firstquarter 2017.

[50] China mobile research institute, "C-RAN: the road towards green RAN", in Proc. C-RAN Int. Workshop, Beijing, China, Apr., 2010.

[51] A. Checko et al., "Cloud RAN for mobile networks: A technology overview," IEEE Commun. Surveys Tuts, vol. 17, no. 1, pp. 405-426, Firstquarter 2015.

[52] S. M. R. Islam, N. Avazov, O. A. Dobre, and K. Kwak, "Power-domain non-orthogonal multiple access (NOMA) in 5G Systems: potentials and challenges," IEEE Commun. Surveys Tuts, vol. 19, no. 2, pp. 721-742, Secondquarter 2017.

[53] A. Sabharwal, P. Schniter, D. Guo, D. W. Bliss, S. Rangarajan, and R. Wichman, "In-band full-duplex wireless: Challenges and opportunities," IEEE J. Sel. Areas Commun., vol. 32, no. 9, pp. 1637-1652, Sep. 2014.

[54] Y. Wu, A. Khisti, C. Xiao, G. Caire, K. Wong, and X. Gao, "A survey of physical layer security techniques for $5 \mathrm{G}$ wireless networks and challenges ahead," IEEE J. Sel. Areas Commun., vol. 36, no. 4, pp. 679-695, Apr. 2018.

[55] B. Blaszczyszyn, M. K. Karray, and H. P. Keeler, "Using Poisson processes to model lattice cellular networks," in Proc. IEEE INFOCOM, pp. 773-781, Turin, Italy, Apr. 2013.

[56] H. P. Keeler, B. Błaszczyszyn, and M. K. Karray, "SINR-based kcoverage probability in cellular networks with arbitrary shadowing," in Proc. IEEE Int. Symp. Inf. Theory, pp. 1167-1171, Istanbul, Turkey 2013.

[57] I. Nakata, N. Miyoshi, "Spatial stochastic models for analysis of heterogeneous cellular networks with repulsively deployed base stations," Perform. Eval., vol. 78, pp. 7-17, Aug. 2014.

[58] P. Madhusudhanan, J. G. Restrepo, Y. Liu, and T. X. Brown, "Analysis of downlink connectivity models in a heterogeneous cellular network via stochastic geometry," IEEE Trans. Wireless Commun., vol. 15, no. 6, pp. 3895-3907, Jun. 2016.

[59] M. Haenggi, "On distances in uniformly random networks," IEEE Trans. Inf. Theory, vol. 51, no. 10, pp. 3584-3586, Oct. 2005.

[60] S. Y. Jung, H. Lee, and S. Kim, "Worst-case user analysis in Poisson Voronoi cells," IEEE Commun. Lett., vol. 17, no. 8, pp. 1580-1583, Aug. 2013.

[61] G. Nigam, P. Minero, and M. Haenggi, "Coordinated multipoint joint transmission in heterogeneous networks," IEEE Trans. Wireless Commun., vol. 62, no. 11, pp. 4134-4146, Nov. 2014.

[62] M. C. Valenti, D. Torrieri, and S. Talarico, "A direct approach to computing spatially averaged outage probability," IEEE Commun. Lett., vol. 18, no. 7, pp. 1103-1106, Jul. 2014.

[63] M. Afshang and H. S. Dhillon, "Fundamentals of modeling finite wireless networks using binomial point process," IEEE Trans. Wireless Commun., vol. 16, no. 5, pp. 3355-3370, May 2017.

[64] J. Guo, S. Durrani, and X. Zhou, "Outage probability in arbitrarilyshaped finite wireless networks," IEEE Trans. Commun., vol. 62, no. 2, pp. 699-712, Feb. 2014.

[65] D. P. Kroese and Z. I. Botev, "Spatial process generation," arXiv preprint, Aug. 2013, [Online]. Available: https://arxiv.org/abs/1308.0399.

[66] D. B. Taylor, H. S. Dhillon, T. D. Novlan, and J. G. Andrews, "Pairwise interaction processes for modeling cellular network topology," in Proc. IEEE Glob. Commun. Conf. (GLOBECOM), pp. 4524-4529, Anaheim, California, USA, Dec. 2012

[67] A. Guo and M. Haenggi, "Spatial stochastic models and metrics for the structure of base stations in cellular networks," IEEE Trans. Wireless Commun., vol. 12, no. 11, pp. 5800-5812, Nov. 2013.

[68] A. Baddeley and R. Turner, "Practical maximum pseudolikelihood for spatial point patterns," Australian and New Zealand J. Stat., vol. 42, pp. 283-322, Sep. 2000.
[69] M. Di Renzo, S. Wang, and X. Xi, "Inhomogeneous double thinningmodeling and analysis of cellular networks by using inhomogeneous Poisson point processes," IEEE Trans. Wireless Commun., vol. 17, no. 8, pp. 5162-5182, Aug. 2018.

[70] M. Di Renzo, A. Guidotti, and G. Corazza, "Average rate of downlink heterogeneous cellular networks over generalized fading channels: A stochastic geometry approach," IEEE Trans. Commun., vol. 61, no. 7, pp. 3050-3071, Jul. 2013.

[71] T. Bai and R. W. Heath Jr., "Coverage and rate analysis for millimeter wave cellular networks," IEEE Trans. Wireless Commun., vol. 14, no. 2, pp. 1100-1114, Oct. 2014.

[72] M. Ding, P. Wang, D. López-Pérez, G. Mao, and Z. Lin, "Performance impact of LoS and NLoS transmissions in dense cellular networks," IEEE Trans. Wireless Commun., vol. 15, no. 3, pp. 2365-2380, Mar. 2016.

[73] I. Atzeni, J. Arnau, and M. Kountouris, "Downlink cellular network analysis with LOS/NLOS propagation and elevated base stations," IEEE Trans. Wireless Commun., vol. 17, no. 1, pp. 142-156, Jan. 2018.

[74] T. Sanguanpuak, S. Guruacharya, E. Hossain, N. Rajatheva, and M. Latva-aho, "Infrastructure sharing for mobile network operators: analysis of trade-offs and market," IEEE Trans. Mobile Comput., vol. 17, no. 12, pp. 2804-2817, Dec. 2018.

[75] V. Sciancalepore, M. Di Renzo, and X. Costa-Perez, "STORNS: stochastic radio access network slicing," in Proc. IEEE Int. Conf. Commun. (ICC), pp. 1-7, Shanghai, China, 2019.

[76] A. K. Gupta, J. G. Andrews, and R. W. Heath, "On the feasibility of sharing spectrum licenses in mmWave cellular systems," IEEE Trans. Commun., vol. 64, no. 9, pp. 3981-3995, Sep. 2016.

[77] A. K. Gupta, A. Alkhateeb, J. G. Andrews, and R. W. Heath, "Gains of restricted secondary licensing in millimeter wave cellular systems," IEEE J. Sel. Areas Commun., vol. 34, no. 11, pp. 2935-2950, Nov. 2016.

[78] J. Park, J. G. Andrews, and R. W. Heath, "Inter-operator base station coordination in spectrum-shared millimeter wave cellular networks," IEEE Trans. Cogn. Commun. Networking, vol. 4, no. 3, pp. 513-528, Sep. 2018.

[79] M. Rebato, M. Mezzavilla, S. Rangan, and M. Zorzi, "Resource sharing in 5G mmWave cellular networks," in Proc. IEEE Int. Conf. Comput. Commun. (INFOCOM Wkshps), pp. 271-276, San Francisco, CA, USA, Apr. 2016.

[80] J. Kibiłda, N. J. Kaminski, and L. A. DaSilva, "Radio access network and spectrum sharing in mobile networks: A stochastic geometry perspective," IEEE Trans. Wireless Commun., vol. 16, no. 4, pp. 25622575, Apr. 2017.

[81] R. Jurdi, A. K. Gupta, J. G. Andrews, and R. W. Heath, "Modeling infrastructure sharing in mmWave networks with shared spectrum licenses," IEEE Trans. Cogn. Commun. and Networking, vol. 4, no. 2, pp. 328-343, Jun. 2018.

[82] V. V. Chetlur and H. S. Dhillon, "Coverage analysis of a vehicular network modeled as Cox process driven by Poisson line process," IEEE Trans Wireless Commun., vol. 17, no. 7, pp. 4401-4416, Jul. 2018.

[83] A. Munari, P. Mahonen, and M. Petrova, "A stochastic geometry approach to asynchronous Aloha full-duplex networks," IEEE/ACM Trans. Netw., vol. 25, no. 6, pp. 3695-3708, Dec. 2017.

[84] A. H. Sakr and E. Hossain, "Cognitive and energy harvesting-based D2D communication in cellular networks: stochastic geometry modeling and analysis," IEEE Trans. Commun., vol. 63, no. 5, pp. 1867-1880, May 2015

[85] Q. Song, X. Lagrange, and L. Nuaymi, "Evaluation of macro diversity gain in long range ALOHA networks," IEEE Commun. Lett., vol. 21, no. 11, pp. 2472-2475, Nov. 2017.

[86] M. Di Renzo, W. Lu, and P. Guan, "The intensity matching approach: A tractable stochastic geometry approximation to system-level analysis of cellular networks," IEEE Trans. Wireless Commun., vol. 15, no. 9, pp. 5963-5983, Sep. 2016

[87] Y. Hmamouche, M. Benjillali, and S. Saoudi, "A Stochastic geometry based approach to tractable 5G RNPO with a new H-LOS model," in Proc. IEEE Wireless Commun. Netw. Conf. (WCNC), Marrakech, Morocco, Apr. 2019.

[88] P. Madhusudhanan, J. G. Restrepo, Y. E. Liu, T. X. Brown, and K. R. Baker, "Stochastic ordering based carrier-to-interference ratio analysis for the shotgun cellular systems," IEEE Wireless Commun. Lett., vol. 1, no. 6, pp. 565-568, Dec. 2012.

[89] P. Madhusudhanan, J. G. Restrepo, Y. Liu, T. X. Brown, and K. R. Baker, "Downlink performance analysis for a generalized shotgun cellular system," IEEE Trans. Wireless Commun., vol. 13, no. 12, pp. 6684-6696, Dec. 2014. 
[90] Y. Li, F. Baccelli, H. S. Dhillon, and J. G. Andrews, "Fitting determinantal point processes to macro base station deployments," in Proc. IEEE Glob. Commun. Conf. (GLOBECOM), pp. 3641-3646, Austin, TX, USA, Dec. 2014.

[91] R. Nasri and A. Jaziri, "Analytical tractability of hexagonal network model with random user location," IEEE Trans. Wireless Commun., vol. 15, no. 5, pp. 3768-3780, May 2016.

[92] M. Michalopoulou, J. Riihijarvi, and P. Mahonen, "Studying the relationships between spatial structures of wireless networks and population densities," in Proc. IEEE Glob. Commun. Conf. (GLOBECOM), pp. 1-6, Miami, FL, USA, Dec. 2010.

[93] H. Ghazzai, E. Yaacoub, M.-S. Alouini, Z. Dawy, and A. Abu-Dayya, "Optimized LTE cell planning with varying spatial and temporal user densities," IEEE Trans. Veh. Technol., vol. 65, no. 3, pp. 1575-1589, Mar. 2016.

[94] A. Achtzehn, J. Riihijarvi, and P. Mahonen, "Large-scale cellular network modeling from population data: an empirical analysis," IEEE Commun. Lett., vol. 20, no. 11, pp. 2292-2295, Nov. 2016.

[95] Y. Zhou et al., "Large-scale spatial distribution identification of base stations in cellular networks," IEEE Access, vol. 3, pp. 2987-2999, 2015.

[96] L. Chiaraviglio et al., "What is the best spatial distribution to model base station density? A deep dive into two european mobile networks," IEEE Access, vol. 4, pp. 1434-1443, 2016.

[97] B. Matérn, "Spatial Variation," Lecture Notes in Statistics, vol. 36, 2nd ed. New York, NY, USA: Springer-Verlag, 1986.

[98] M. Haenggi, "Mean Interference in hard-core wireless networks," IEEE Commun. Lett., vol. 15, no. 8, pp. 792-794, Aug. 2011.

[99] B. Cho, K. Koufos, and R. Jantti, "Bounding the mean interference in Matérn type II hard-core wireless networks," IEEE Wireless Commun. Lett., vol. 2, no. 5, pp. 563-566, Oct. 2013.

[100] A. Al-Hourani, R. J. Evans, and S. Kandeepan, "Nearest neighbor distance distribution in hard-core point processes," IEEE Commun. Lett., vol. 20, no. 9, pp. 1872-1875, Sep. 2016.

[101] F. J. Martín-Vega, B. Soret, M. C. Aguayo-Torres, I. Z. Kovács, and G. Gómez, "Geolocation-based access for vehicular communications: analysis and optimization via stochastic geometry," IEEE Trans. Veh. Technol., vol. 67, no. 4, pp. 3069-3084, Apr. 2018

[102] F. Lagum, S. S. Szyszkowicz, and H. Yanikomeroglu, "CoV-based metrics for quantifying the regularity of hard-core point processes for modeling base station locations," IEEE Wireless Commun. Lett., vol. 5, no. 3, pp. 276-279, Jun. 2016.

[103] M.N.M. van Lieshout, Markov Point Processes and their Applications, Imperial College Press, London, 2000.

[104] I. Flint, H.-B. Kong, N. Privault, P. Wang, and D. Niyato, "Analysis of heterogeneous wireless networks using Poisson hard-core hole process," IEEE Trans. Wireless Commun., vol. 16, no. 11, pp. 71527167, Nov. 2017.

[105] C. Lee and M. Haenggi, "Interference and outage in Poisson cognitive networks," IEEE Trans. Wireless Commun., vol. 11, no. 4, pp. 13921401, Apr. 2012.

[106] N. Deng, W. Zhou, and M. Haenggi, "Heterogeneous cellular network models with dependence," IEEE J. Sel. Areas Commun., vol. 33, no. 10, pp. 2167-2181, Oct. 2015.

[107] Z. Chen and M. Kountouris, "Decentralized opportunistic access for D2D underlaid cellular networks," IEEE Trans. Commun., vol. 66, no. 10, pp. 4842-4853, Oct. 2018

[108] Z. Yazdanshenasan, H. S. Dhillon, M. Afshang, and P. H. J. Chong, "Poisson hole process: Theory and applications to wireless networks," IEEE Trans. Wireless Commun., vol. 15, no. 11, pp. 7531-7546, Nov. 2016

[109] R. K. Ganti, F. Baccelli, and J. G. Andrews, "Series expansion for interference in wireless networks," IEEE Trans. Inf. Theory, vol. 58, no. 4, pp. 2194-2205, Apr. 2012.

[110] Y. Li, F. Baccelli, H. S. Dhillon, and J. G. Andrews, "Statistical modeling and probabilistic analysis of cellular networks with determinantal point processes," IEEE Trans. Commun., vol. 63, no. 9, pp. 3405-3422, Sep. 2015.

[111] N. Miyoshi and T. Shirai, "A cellular network model with Ginibre configurated base stations," Research Rep. on Math. and Comp. Sciences (Tokyo Inst. of Tech.), Oct. 2012.

[112] I. Nakata and N. Miyoshi, "Spatial stochastic models for analysis of heterogeneous cellular networks with repulsively deployed base stations," Tokyo Institute of Technology Preprint, Oct. 2013.

[113] N. Deng, W. Zhou, and M. Haenggi, "The Ginibre point process as a model for wireless networks with repulsion," IEEE Trans. Wireless Commun., vol. 14, no. 1, pp. 107-121, Jan. 2015.
[114] H.-B. Kong, P. Wang, D. Niyato, and Y. Cheng, "Physical layer security in wireless networks with Ginibre point processes," IEEE Trans. Wireless Commun., vol. 17, no. 8, pp. 5132-5147, May 2018.

[115] F. Lavancier, J. Møller, and E. Rubak, "Statistical aspects of determinantal point processes," arXiv preprint, Jun. 2014, [Online]. Available: https://arxiv.org/abs/1205.4818.

[116] S. A. Banani, A. W. Eckford, and R. S. Adve, "The penalty for random deployment in hexagonal lattice networks with perturbed interferers," in Proc. IEEE Globecom Workshops, pp. 1272-1277, Austin, TX, USA, Dec. 2014.

[117] S. A. Banani, R. S. Adve, and A. W. Eckford, "A perturbed hexagonal lattice to model basestation locations in real-world cellular networks," in Proc. IEEE Globecom Workshops, pp. 1-6, San Diego, CA, USA, Dec. 2015

[118] F. Lagum, S. S. Szyszkowicz, and H. Yanikomeroglu, "Quantifying the regularity of perturbed triangular lattices using CoV-Based metrics for modeling the locations of base stations in HetNets," in Proc. IEEE 84th Veh. Technol. Conf. (VTC-Fall), pp. 1-5, Montreal, QC, Canada, 2016.

[119] Q.-N. Le-The, T. Beitelmal, F. Lagum, S. S. Szyszkowicz, and H. Yanikomeroglu, "Cell switch-off algorithms for spatially irregular base station deployments," IEEE Wireless Commun. Lett., vol. 6, no. 3, pp. 354-357, Apr. 2017.

[120] F. Lagum, Q.-N. Le-The, T. Beitelmal, S. S. Szyszkowicz, H. Yanikomeroglu, "Cell switch-off for networks deployed with variable spatial regularity," IEEE Wireless Commun. Lett., vol. 6, no. 2, pp. 234-237, Feb. 2017.

[121] M. Mirahsan, R. Schoenen and H. Yanikomeroglu, "HetHetNets: heterogeneous traffic distribution in heterogeneous wireless cellular networks," IEEE J. Sel. Areas Commun., vol. 33, no. 10, pp. 22522265, Oct. 2015

[122] M. Tanemura, "Statistical distributions of poisson Voronoi cells in two and three dimensions," Forma, vol. 18, no. 4, pp. 221-247, Nov. 2003.

[123] C. Choi, J. O. Woo, and J. G. Andrews, "An analytical framework for modeling a spatially repulsive cellular network," IEEE Trans. on Commun., vol. 66, no. 2, pp. 862-874, Feb. 2018.

[124] R. K. Ganti and M. Haenggi, "Interference and outage in clustered wireless AdHoc networks," IEEE Trans. Inf. Theory, vol. 55, no. 9, pp. 4067-4086, Sep. 2009.

[125] Y. J. Chun, M. O. Hasna, and A. Ghrayeb, "Modeling heterogeneous cellular networks interference using Poisson cluster processes," IEEE J. Sel. Areas Commun., vol. 33, no. 10, pp. 2182-2195, Oct. 2015.

[126] C. Saha, M. Afshang, and H. S. Dhillon, "Enriched K-tier HetNet model to enable the analysis of user-centric small cell deployments," IEEE Trans. Wireless Commun., vol. 16, no. 3, pp. 1593-1608, Mar. 2017.

[127] C. Saha, M. Afshang, and H. S. Dhillon, "3GPP-inspired HetNet model using Poisson cluster process: sum-product functionals and downlink coverage," IEEE Trans. Commun., vol. 66, no. 5, pp. 2219-2234, May 2018.

[128] P. D. Mankar, G. Das, and S. S. Pathak, "Modeling and coverage analysis of BS-centric clustered users in a random wireless network," IEEE Wireless Commun. Lett., vol. 5, no. 2, pp. 208-211, Apr. 2016.

[129] M. Afshang and H. S. Dhillon, "Poisson cluster process based analysis of HetNets with correlated user and base station locations," IEEE Trans. Wireless Commun., vol. 17, no. 4, pp. 2417-2431, Apr. 2018.

[130] R. Li, Z. Zhao, Y. Zhong, C. Qi, and H. Zhang, "The stochastic geometry analyses of cellular networks with $\alpha$-stable self-similarity," IEEE Trans. Commun., vol. 67, no. 3, pp. 2487-2503, Mar. 2019.

[131] Y. Zhou, R. Li, Z. Zhao, X. Zhou, and H. Zhang, "On the $\alpha$-stable distribution of base stations in cellular networks," IEEE Commun. Lett., vol. 19, no. 10, pp. 1750-1753, Oct. 2015.

[132] J. Møller, "Shot noise Cox processes," Adv. Appl. Probab., vol. 35, no. 3, pp. 4-26, Sep. 2003.

[133] C. Choi and F. Baccelli, "Poisson Cox point processes for vehicular networks," IEEE Trans. Veh. Technol., vol. 67, no. 10, pp. 10160-10165, Oct. 2018.

[134] C. Choi and F. Baccelli, "An analytical framework for coverage in cellular networks leveraging vehicles," IEEE Trans. Commun., vol. 66 , no. 10, pp. 4950-4964, Oct. 2018.

[135] Z. Wang, R. Schoenen, H. Yanikomeroglu, and M. St-Hilaire, "The impact of user spatial heterogeneity in heterogeneous cellular networks," in Proc. IEEE Globecom Workshops (GC Wkshps), pp. 1278-1283, Austin, TX, USA, Dec. 2014

[136] J. Kibiłda, B. Galkin, and L. A. DaSilva, "Modelling multi-operator base station deployment patterns in cellular networks," IEEE Trans. Mobile Comput., vol. 15, no. 12, pp. 3087-3099, Dec. 2016. 
[137] Q. Cui, N. Wang, and M. Haenggi, "Vehicle distributions in large and small cities: spatial models and applications," IEEE Trans. Veh. Technol., vol. 67, no. 11, pp. 10176-10189, Nov. 2018.

[138] A. Guo, Y. Zhong, W. Zhang, and M. Haenggi, "The Gauss-Poisson process for wireless networks and the benefits of cooperation," IEEE Trans. Commun., vol. 64, no. 5, pp. 1916-1929, May 2016.

[139] N. Deng and M. Haenggi, "The benefits of hybrid caching in GaussPoisson D2D networks," IEEE J. Sel. Areas Commun., vol. 36, no. 6, pp. 1217-1230, Jun. 2018.

[140] H. S. Dhillon, R. K. Ganti, and J. G. Andrews, "Modeling nonuniform UE distributions in downlink cellular networks," IEEE Wireless Commun. Lett., vol. 2, no. 3, pp. 339-342, Jun. 2013.

[141] H. S. Dhillon, R. K. Ganti, and J. G. Andrews, "Load-aware modeling and analysis of heterogeneous cellular networks," IEEE Trans. Wireless Commun., vol. 12, no. 4, pp. 1666-1677, Apr. 2013.

[142] S. Lee and K. Huang, "Coverage and economy of cellular networks with many base stations," IEEE Commun. Lett., vol. 16, no. 7, pp. 1038-1040, Jul. 2012.

[143] M. Haenggi, "The mean interference-to-signal ratio and its key role in cellular and amorphous networks," IEEE Wireless Commun. Lett., pp. 597-600, Dec. 2014.

[144] A. Guo and M. Haenggi, "Asymptotic deployment gain: A simple approach to characterize the SINR distribution in general cellular networks," IEEE Trans. Commun., vol. 63, pp. 962-976, Mar. 2015.

[145] R. K. Ganti and M. Haenggi, "Asymptotics and approximation of the SIR distribution in general cellular networks," IEEE Trans. Wireless Commun., vol. 15, no. 3, pp. 2130-2143, Mar. 2016.

[146] B. Błaszczyszyn and D. Yogeshwaran, "Directionally convex ordering of random measures, shot-noise fields and some applications to wireless networks," Adv. Appl. Prob., vol. 41, pp. 623-646, Jun. 2009.

[147] J. Lee and C. Tepedelenlioglu, "Stochastic ordering of interference in large-scale wireless networks," IEEE Trans. Signal Process., vol. 62, no. 3, pp. 729-740, Feb., 2014.

[148] M. Van Lieshout and A. Baddeley, "A nonparametric measure of spatial interaction in point patterns," Statistica Neerlandica, vol. 50, pp. 344361, 1996

[149] C. Chen, R. C. Elliott, W. A. Krzymień, and J. Melzer, "Modeling of cellular networks using stationary and nonstationary point processes," IEEE Access, vol. 6, pp. 47144-47162, 2018.

[150] A. Okabe, B. Boots, K. Sugihara, and S. N. Chiu, Spatial Tessellations: Concepts and Applications of Voronoi Diagrams, 2nd Ed..Chichester, West Sussex, England: John Wiley \& Sons, 1999.

[151] S. Krishnan and H. S. Dhillon, "Spatio-temporal interference correlation and joint coverage in cellular networks," IEEE Trans. Wireless Commun., vol. 16, no. 9, pp. 5659-5672, Sep. 2017.

[152] H. ElSawy and E. Hossain, "On stochastic geometry modeling of cellular uplink transmission with truncated channel inversion power control," IEEE Trans. Wireless Commun., vol. 13, no. 8, pp. 44544469, Aug. 2014.

[153] M. Di Renzo and P. Guan, "Stochastic geometry modeling and systemlevel analysis of uplink heterogeneous cellular networks with multiantenna base stations," IEEE Trans. Commun., vol. 64, no. 6, pp. 2453 2476, Jun. 2016.

[154] F. J. Martin-Vega, G. Gomez, M. C. Aguayo-Torres, and M. Di Renzo, "Analytical modeling of interference aware power control for the uplink of heterogeneous cellular networks," IEEE Trans. Wireless Commun., vol. 15 , no. 10, pp. 6742-6757, Oct. 2016.

[155] P. Herath, C. Tellambura, and W. A. Krzymien, "Stochastic geometry modeling of cellular uplink power control under composite RayleighLognormal fading," in Proc. IEEE 82nd Veh. Technol. Conf. (VTC2015Fall), pp. 1-5, Boston, MA, 2015.

[156] S. Singh, X. Zhang, and J. G. Andrews, "Joint rate and SINR coverage analysis for decoupled uplink-downlink biased cell associations in HetNets," IEEE Trans. Wireless Commun., vol. 14, no. 10, pp. 53605373, Oct. 2015

[157] L. Zhang, W. Nie, G. Feng, F. Zheng, and S. Qin, "Uplink performance improvement by decoupling uplink/downlink access in HetNets," IEEE Trans. Veh. Technol., vol. 66, no. 8, pp. 6862-6876, Aug. 2017.

[158] F. Boccardi et al., "Why to decouple the uplink and downlink in cellular networks and how to do it," IEEE Commun. Mag., vol. 54, no. 3, pp. 110-117, Mar. 2016

[159] M. Haenggi, "User point processes in cellular networks," IEEE Wireless Commun. Lett., vol. 6, no. 2, pp. 258-261, Apr. 2017.

[160] M. Afshang, H. S. Dhillon, and P. H. Joo Chong, "Modeling and performance analysis of clustered device-to-device networks," IEEE Trans. Wireless Commun., vol. 15, no. 7, pp. 4957-4972, Jul. 2016.
[161] M. Afshang, H. S. Dhillon, and P. H. J. Chong, "Fundamentals of cluster-centric content placement in cache-enabled device-to-device networks," IEEE Trans. Commun., vol. 64, no. 6, pp. 2511-2526, Jun. 2016.

[162] W. Yi, Y. Liu, and A. Nallanathan, "Modeling and analysis of D2D millimeter-wave networks with Poisson cluster processes," IEEE Trans. Commun., vol. 65, no. 12, pp. 5574-5588, Dec. 2017.

[163] X. Lin, J. G. Andrews, and A. Ghosh, "Spectrum sharing for deviceto-device communication in cellular networks," IEEE Trans. Wireless Commun., vol. 13, no. 12, pp. 6727-6740, Dec. 2014.

[164] S. Singh, M. N. Kulkarni, A. Ghosh, and J. G. Andrews, "Tractable model for rate in self-backhauled millimeter wave cellular networks," IEEE J. Sel. Areas Commun., vol. 33, no. 10, pp. 2196-2211, Oct. 2015.

[165] M. Di Renzo, "Stochastic geometry modeling and analysis of multi-tier millimeter wave cellular networks," IEEE Trans. Wireless Commun., vol. 14, no. 9, pp. 5038-5057, Sep. 2015.

[166] T. Bai, R. Vaze, and R. W. Heath, "Analysis of blockage effects on urban cellular networks," IEEE Trans. Wireless Commun., vol. 13, no. 9, pp. 5070-5083, Sep. 2014.

[167] H. Inaltekin, M. Chiang, H. V. Poor, and S. B. Wicker, "On unbounded path-loss models: effects of singularity on wireless network performance," IEEE J. Sel. Areas Commun., vol. 27, no. 7, pp. 1078-1092, Sep. 2009.

[168] X. Zhang and J. G. Andrews, "Downlink cellular network analysis with multi-slope path loss models," IEEE Trans. Commun., vol. 63, no. 5, pp. 1881-1894, May 2015.

[169] J. G. Andrews, X. Zhang, G. D. Durgin, and A. K. Gupta, "Are we approaching the fundamental limits of wireless network densification?," IEEE Commun. Mag., vol. 54, no. 10, pp. 184-190, Oct. 2016.

[170] J. Liu, M. Sheng, L. Liu, and J. Li, "Effect of densification on cellular network performance with bounded pathloss model," IEEE Commun. Lett., vol. 21, no. 2, pp. 346-349, Feb. 2017.

[171] A. AlAmmouri, J. G. Andrews, and F. Baccelli, "SINR and throughput of dense cellular networks with stretched exponential path loss," IEEE Trans. on Wireless Commun., vol. 17, no. 2, pp. 1147-1160, Feb. 2018

[172] J. G. Andrews, T. Bai, M. N. Kulkarni, A. Alkhateeb, A. K. Gupta, and R. W. Heath, "Modeling and analyzing millimeter wave cellular systems," IEEE Trans. Commun., vol. 65, no. 1, pp. 403-430, Jan. 2017.

[173] A. Al-Hourani, S. Kandeepan, and S. Lardner, "Optimal LAP altitude for maximum coverage," IEEE Wireless Commun. Lett., vol. 3, no. 6, pp. 569-572, Dec. 2014

[174] A. A. Khuwaja, Y. Chen, N. Zhao, M.-S. Alouini, and P. Dobbins, "A survey of channel modeling for UAV communications," IEEE Commun. Surveys Tuts, vol. 20, no. 4, pp. 2804-2821, Fourthquarter 2018.

[175] J. Liu, M. Sheng, L. Liu, and J. Li, "Performance of small cell networks under multislope bounded pathloss model: from sparse to ultradense deployment," IEEE Trans. Veh. Technol., vol. 67, no. 11, pp. 11022 11034, Nov. 2018.

[176] C. Galiotto, N. K. Pratas, N. Marchetti, and L. Doyle, "A stochastic geometry framework for LOS/NLOS propagation in dense small cell networks," in Proc. IEEE Int. Conf. Commun. (ICC), pp. 2851-2856, London, UK, 2015.

[177] M. Ding, and D. López-Pérez, "Performance impact of base station antenna heights in dense cellular networks," IEEE Trans. Wireless Commun., vol. 16, no. 12, pp. 8147-8161, Dec. 2017.

[178] Z. Zhang and R. Qingyang Hu, "Dense cellular network analysis with LoS/NLoS propagation and bounded path loss model," IEEE Commun. Lett., vol. 22, no. 11, pp. 2386-2389, Nov. 2018.

[179] Q. Ye, B. Rong, Y. Chen, M. Al-Shalash, C. Caramanis, and J. G. Andrews, "User association for load balancing in heterogeneous cellular networks,"IEEE Trans. Wireless Commun., vol. 12, no. 6, pp. 2706-2716, Jun. 2013.

[180] S. Singh, H. S. Dhillon, and J. G. Andrews, "Offloading in heterogeneous networks: modeling, analysis, and design insights," IEEE Trans. Wireless Commun., vol. 12, no. 5, pp. 2484-2497, May 2013.

[181] S. Singh and J. G. Andrews, "Joint resource partitioning and offloading in heterogeneous cellular networks," IEEE Trans. Wireless Commun., vol. 13, no. 2, pp. 888-901, Feb. 2014.

[182] A. H. Sakr and E. Hossain, "On user association in multi-tier fullduplex cellular networks," IEEE Trans. Commun., vol. 65, no. 9, pp. 4080-4095, Sep. 2017.

[183] I. Rhee, M. Shin, S. Hong, K. Lee, S. J. Kim, and S. Chong, "On the Levy-walk nature of human mobility," IEEE/ACM Trans. on Netw., vol. 19 , no. 3, pp. 630-643, Jun. 2011. 
[184] J. B. T. Camp and V. Davies, "A survey of mobility models for adhoc network research," Wireless Commun. Mobile Comput. Special Issue on Mobile Ad Hoc Netw.: Research, Trends and Applications, vol. 2, no. 5, pp. 483-502, 2002.

[185] X. Lin, R. K. Ganti, P. J. Fleming, and J. G. Andrews, "Towards understanding the fundamentals of mobility in cellular networks," IEEE Trans. Wireless Commun., vol. 12, no. 4, pp. 1686-1698, Apr. 2013.

[186] W. Bao and B. Liang, "Stochastic geometric analysis of user mobility in heterogeneous wireless networks," IEEE J. Sel. Areas Commun., vol. 33, no. 10, pp. 2212-2225, Oct. 2015

[187] X. Xu, Z. Sun, X. Dai, T. Svensson, and X. Tao, "Modeling and analyzing the cross-tier handover in heterogeneous networks," IEEE Trans. Wireless Commun., vol. 16, no. 12, pp. 7859-7869, Dec. 2017

[188] X. Ge, J. Ye, Y. Yang, and Q. Li, "User mobility evaluation for 5G small cell networks based on individual mobility model," IEEE J. Sel. Areas Commun., vol. 34, no. 3, pp. 528-541, Mar. 2016.

[189] R. Arshad, H. ElSawy, S. Sorour, T. Y. Al-Naffouri, and M.-S. Alouini, "Velocity-aware handover management in two-tier cellular networks," IEEE Trans. Wireless Commun., vol. 16, no. 3, pp. 1851-1867, Mar. 2017

[190] S. Sadr and R. S. Adve, "Handoff rate and coverage analysis in multitier heterogeneous networks," IEEE Trans. Wireless Commun., vol. 14, no. 5, pp. 2626-2638, May 2015.

[191] S. Hsueh and K. Liu, "An equivalent analysis for handoff probability in heterogeneous cellular networks," IEEE Commun. Lett., vol. 21, no. 6, pp. 1405-1408, Jun. 2017.

[192] Y. Hmamouche, M. Benjillali, S. Saoudi, and H. Yanikomeroglu, "Uplink coverage and handoff rate with realistic power control models and blind cell search," in Proc. IEEE Int. Pers., Indoor Mobile Radio Commun. Symp. (PIMRC), pp. 1-7, London, United Kingdom, Aug. 2020

[193] H. Tabassum, M. Salehi and E. Hossain, "Fundamentals of MobilityAware Performance Characterization of Cellular Networks: A Tutorial," IEEE Commun. Surveys Tuts, vol. 21, no. 3, pp. 2288-2308, Thirdquarter 2019.

[194] S. Enayati, H. Saeedi, H. Pishro-Nik, and H. Yanikomeroglu, "Moving aerial base station networks: A stochastic geometry analysis and design perspective," IEEE Trans. Wireless Commun., vol. 18, no. 6, pp. 29772988, Jun. 2019.

[195] V. V. Chetlur and H. S. Dhillon, "Downlink coverage analysis for a finite 3-D wireless network of unmanned aerial vehicles," IEEE Trans. Commun., vol. 65, no. 10, pp. 4543-4558, Oct. 2017.

[196] M. Banagar and H. S. Dhillon, "Performance characterization of canonical mobility models in drone cellular networks," IEEE Trans. Wireless Commun., vol. 19, no. 7, pp. 4994-5009, Jul. 2020.

[197] Y. Sui, J. Vihriala, A. Papadogiannis, M. Sternad, W. Yang, and T. Svensson, "Moving cells: a promising solution to boost performance for vehicular users," IEEE Commun. Mag., vol. 51, no. 6, pp. 62-68, Jun. 2013.

[198] X. Tang, X. Xu, T. Svensson, and X. Tao, "Coverage performance of joint transmission for moving relay enabled cellular networks in dense urban scenarios," IEEE Access, vol. 5, pp. 13001-13009, Jul. 2017.

[199] X. Ge et al., "Wireless fractal cellular networks," IEEE Wireless Commun., vol. 23, no. 5, pp. 110-119, Oct. 2016.

[200] J. Chen, X. Ge, and Q. Ni, "Coverage and handoff analysis of 5G fractal small cell networks," IEEE Trans. Wireless Commun., vol. 18, no. 2, pp. 1263-1276, Feb. 2019.

[201] Y. Zhong, T. Q. S. Quek, and X. Ge, "Heterogeneous cellular networks with spatio-temporal traffic: delay analysis and scheduling," IEEE $J$. Sel. Areas Commun., vol. 35, no. 6, pp. 1373-1386, Jun 2017.

[202] M. Salehi, A. Mohammadi, and M. Haenggi, "Analysis of D2D underlaid cellular networks: SIR Meta distribution and mean local delay," IEEE Trans. Commun., vol. 65, pp. 2904-2916, Jul. 2017.

[203] Y. Zhong, M. Haenggi, F. Zheng, W. Zhang, T. Q. S. Quek, and W. Nie, "Toward a tractable delay analysis in ultra-dense networks," IEEE Commun. Mag., vol. 55, no. 12, pp. 103-109, Dec. 2017.

[204] D. C. Chen, T. Q. S. Quek, and M. Kountouris, "Backhauling in heterogeneous cellular networks: modeling and tradeoffs," IEEE Trans. Wireless Commun., vol. 14, no. 6, pp. 3194-3206, Jun. 2015

[205] M. A. Abd-Elmagid, N. Pappas, and H. S. Dhillon, "On the role of age of information in the internet of things," IEEE Commun. Mag., vol. 57, no. 12, pp. 72-77, Dec. 2019.

[206] Y. Hu, Y. Zhong, and W. Zhang, "Age of information in Poisson networks," in Proc. 10th Int. Conf. Wireless Commun. Signal Process. (WCSP), Oct. 2018, pp. 1-6.
[207] M. Emara, H. Elsawy, and G. Bauch, "A spatiotemporal model for peak AoI in uplink IoT networks: time versus event-triggered traffic," IEEE Internet Things J., vol. 7, no. 8, pp. 6762-6777, Aug. 2020.

[208] H. H. Yang, A. Arafa, T. Q. Quek, and V. Poor, "Optimizing information freshness in wireless networks: A stochastic geometry approach," IEEE Trans. Mobile Comput., doi: 10.1109/TMC.2020.2977010.

[209] X. Lu, M. Salehi, M. Haenggi, E. Hossain, and H. Jiang, "Stochastic geometry analysis of spatial-temporal performance in wireless networks: a tutorial," arXiv preprint, Feb. 2021, [Online]. Available: https://arxiv.org/abs/2102.00588.

[210] C. E. Shannon, "Two-way communication channels," in Proc. 4th Berkeley Symp. Math. Statist. And Prob., vol. 1, pp. 611-644, 1961.

[211] P. Chang and G. Miao, "Energy and spectral efficiency of cellular networks with discontinuous transmission," IEEE Trans. Commun., vol. 16, n.o5, pp.2991-3002, May 2017.

[212] M. Haenggi, "The Meta distribution of the SIR in Poisson bipolar and cellular networks," IEEE Trans. Wireless Commun., vol. 15, no. 4, pp. 2577-2589, Apr. 2016.

[213] H. Ji, S. Park, J. Yeo, Y. Kim, J. Lee, and B. Shim, "Ultra-reliable and low-latency communications in 5G downlink: physical layer aspects," IEEE Wireless Commun., vol. 25, no. 3, pp. 124-130, Jun. 2018.

[214] I. Ramezanipour, P. Nouri, H. Alves, P. H. J. Nardelli, R. D. Souza, and A. Pouttu, "Finite blocklength communications in smart grids for dynamic spectrum access and locally licensed scenarios," IEEE Sensors J., vol. 18, no. 13, pp. 5610-5621, Jul. 2018.

[215] Y. Polyanskiy, H. V. Poor, and S. Verdu, "Channel coding rate in the finite blocklength regime," IEEE Trans. Inf. Theory, vol. 56, no. 5, pp. 2307-2359, May 2010.

[216] M.-S. Alouini and A. J. Goldsmith, "Area spectral efficiency of cellular mobile radio systems," IEEE Trans. Veh. Technol., vol. 48, no. 4, pp. 1047-1066, Jul. 1999.

[217] C. Li, J. Zhang, J. G. Andrews, and K. B. Letaief, "Success probability and area Spectral efficiency in multiuser MIMO hetNets," IEEE Trans. on Commun., vol. 64, no. 4, pp. 1544-1556, Apr. 2016.

[218] M. Di Renzo, A. Zappone, T. T. Lam, and M. Debbah, "System-level modeling and optimization of the energy efficiency in cellular networks - A stochastic geometry framework," IEEE Trans. Wireless Commun., vol. 17, no. 4, pp. 2539-2556, Apr. 2018.

[219] M. Di Renzo, T. T. Lam, A. Zappone, and M. Debbah, ”A tractable closed-form expression of the coverage probability in Poisson cellular networks," IEEE Wireless Commun. Lett., vol. 8, no. 1, pp. 249-252, Feb. 2019.

[220] Y. Deng, L. Wang, M. Elkashlan, M. Di Renzo, and J. Yuan, "Modeling and analysis of wireless power transfer in heterogeneous cellular networks," IEEE Trans. Commun., vol. 64, no. 12, pp. 5290-5303, Dec. 2016.

[221] M. Di Renzo and W. Lu, "System-level analysis and optimization of cellular networks with simultaneous wireless information and power transfer: stochastic geometry modeling," IEEE Trans. Veh. Technol., vol. 66, no. 3, pp. 2251-2275, Mar. 2017.

[222] S. Guruacharya, H. Tabassum, and E. Hossain, "Integral approximations for coverage probability," IEEE Wireless Commun. Lett., vol. 5 , no. 1, pp. 24-27, Feb. 2016.

[223] Y. Hmamouche, M. Benjillali, and S. Saoudi, "Stochastic analysis of UDNs with resource capacity and user scheduling," in Proc. IEEE Wireless Commun. and Netw. Conf. (WCNC), Marrakech, Morocco, Apr. 2019.

[224] S. Guruacharya and E. Hossain, "Approximation of Meta distribution and its moments for Poisson cellular networks," IEEE Wireless Commun. Lett., vol. 7, no. 6, pp. 1074-1077, Dec. 2018.

[225] M. Haenggi, "Efficient calculation of Meta distributions and the performance of user percentiles," IEEE Wireless Commun. Lett., vol. 7 , no. 6, pp. 982-985, Dec. 2018.

[226] S. S. Kalamkar and M. Haenggi, "Simple approximations of the SIR meta distribution in general cellular networks," IEEE Trans. Commun., vol. 67, no. 6, pp. 4393-4406, Jun. 2019.

[227] N. Deng and M. Haenggi, "A fine-grained analysis of millimeter-wave device-to-device networks," IEEE Trans. Commun., vol. 65, no. 11, pp. 4940-4954, Nov. 2017.

[228] Q. Cui, X. Yu, Y. Wang, and M. Haenggi, "The SIR Meta distribution in Poisson cellular networks with base station cooperation," IEEE Trans. Commun., vol. 66, pp. 1234-1249, Mar. 2018.

[229] Y. Wang, M. Haenggi, and Z. Tan, "The Meta distribution of the SIR for cellular networks with power control," IEEE Trans. Commun., vol. 66, no. 4, pp. 1745-1757, Apr. 2018. 
[230] Q. Cui, X. Yu, Y. Wang, and M. Haenggi, "The SIR Meta distribution in Poisson cellular networks with base station cooperation," IEEE Trans. Commun., vol. 66, no. 3, pp. 1234-1249, Mar. 2018.

[231] J. Tang, G. Chen, and J. P. Coon, "Meta distribution of the secrecy rate in the presence of randomly located eavesdroppers," IEEE Wireless Commun. Lett., vol. 7, no. 4, pp. 630-633, Aug. 2018.

[232] N. Deng and M. Haenggi, "The Energy and rate Meta distributions in wirelessly powered D2D networks," IEEE J. Sel. Areas Commun., vol. 37, no. 2, pp. 269-282, Feb. 2019.

[233] K. S. Ali, H. El Sawy, and M.-S. Alouini, "Meta distribution of downlink non-orthogonal multiple access (NOMA) in Poisson networks," IEEE Wireless Commun. Lett., vol. 8, no. 2, pp. 572-575, Apr. 2019.

[234] X. Wang, H. Zhang, Y. Tian, and V. C. M. Leung, "Modeling and analysis of aerial base station-assisted cellular networks in finite areas under LoS and NLoS propagation," IEEE Trans. Wireless Commun., vol. 17, no. 10, pp. 6985-7000, Oct. 2018.

[235] Z. Khalid and S. Durrani, "Distance distributions in regular polygons," IEEE Trans. Veh. Technol., vol. 62, no. 5, pp. 2363-2368, Jun. 2013.

[236] C. Tepedelenlioglu, A. Abdi, and G. B. Giannakis, "The Ricean K factor: estimation and performance analysis," IEEE Trans. Wireless Commun., vol. 2, no. 4, pp. 799-810, Jul. 2003.

[237] O. Georgiou, "Polarized rician fading models for performance analysis in cellular networks," IEEE Commun. Lett., vol. 20, no. 6, pp. 12551258, Jun. 2016.

[238] A. M. Hunter, J. G. Andrews, and S. Weber, "Transmission capacity of ad hoc networks with spatial diversity," IEEE Trans. Wireless Commun., vol. 7, no. 12, pp. 5058-5071, Dec. 2008.

[239] A. K. Gupta, H. S. Dhillon, S. Vishwanath, and J. G. Andrews, "Downlink multi-antenna heterogeneous cellular network with load balancing," IEEE Trans. Commun., vol. 62, no. 11, pp. 4052-4067, Nov. 2014.

[240] M. Bacha, Y. Wu, and B. Clerckx, "Downlink and uplink decoupling in two-tier heterogeneous networks with multi-antenna base stations," IEEE Trans. Wireless Commun., vol. 16, no. 5, pp. 2760-2775, May 2017.

[241] R. Tanbourgi, H. S. Dhillon, and F. K. Jondral, "Analysis of joint transmit-receive diversity in downlink MIMO heterogeneous cellular networks," IEEE Trans. Wireless Commun., vol. 14, no. 12, pp. 66956709, Dec. 2015.

[242] W. P. Johnson, "The curious history of Faa di Bruno's formula," Amer. Math. Monthly, vol. 109, no. 3, pp. 217-234, Mar. 2002.

[243] C. Li, J. Zhang, and K. B. Letaief, "Throughput and energy efficiency analysis of small cell networks with multi-antenna base stations," IEEE Trans. Wireless Commun., vol. 13, no. 5, pp. 2505-2517, May 2014.

[244] S. Mukherjee, "Downlink SINR distribution in a heterogeneous cellular wireless network with max-SINR connectivity," in Proc. 49th Annual Allerton Conf. Commun., Control, and Comput. (Allerton), pp. 16491656, Monticello, IL, USA, 2011.

[245] S. Mukherjee, "Distribution of downlink SINR in heterogeneous cellular networks," IEEE J. Sel. Areas Commun., vol. 30, no. 3, pp. 575-585, Apr. 2012.

[246] B. Błaszczyszyn and H. P. Keeler, "Studying the SINR process of the typical user in Poisson networks using its factorial moment measures," IEEE Trans. Inf. Theory, vol. 61, no. 12, pp. 6774-6794, Dec. 2015.

[247] J. Gil-Pelaez, "Note on the inversion theorem," Biometrika, vol. 38, no. 3/4, pp. 481-482, Dec. 1951.

[248] M. Di Renzo and P. Guan, "Stochastic geometry modeling of coverage and rate of cellular networks using the Gil-Pelaez inversion theorem," IEEE Commun. Lett., vol. 18, no. 9, pp. 1575-1578, Sep. 2014.

[249] Y. Kim, T. Lee, and D. I. Kim, "Joint information and power transfer in SWIPT-enabled CRFID networks," IEEE Wireless Commun. Lett., vol. 7, no. 2, pp. 186-189, Apr. 2018

[250] M. G. Khoshkholgh and V. C. M. Leung, "Coverage analysis of MaxSIR cell association in HetNets under Nakagami fading," IEEE Trans. Veh. Technol., vol. 67, no. 3, pp. 2420-2438, Mar. 2018.

[251] B. Yang, M. Ding, G. Mao, and X. Ge, "Performance analysis of dense small cell networks with generalized fading," in Proc. IEEE Int. Conf. Commun. (ICC), pp. 1-7, Paris, France, 2017.

[252] M. Win, P. Pinto, and L. Shepp, "A mathematical theory of network interference and its applications," Proc. IEEE, vol. 97, no. 2, pp. 205230, Feb. 2009.

[253] R. W. Heath, M. Kountouris, and T. Bai, "Modeling heterogeneous network interference using Poisson point processes," IEEE Trans. Sig. Process., vol. 61, no. 16, pp. 4114-4126, Aug. 2013.

[254] K. Hamdi, "A useful lemma for capacity analysis of fading interference channels," IEEE Trans. Commun., vol. 58, no. 2, pp. 411-416, Feb. 2010.
[255] H. Tabassum, F. Yilmaz, Z. Dawy, and M.-S. Alouini, "A framework for uplink intercell interference modeling with channel-based scheduling," IEEE Trans. Wireless Commun., vol. 12, no. 1, pp. 206-217, Jan. 2013.

[256] M. Kamel, W. Hamouda, and A. Youssef, "Performance analysis of multiple association in ultra-dense networks," IEEE trans. Commun., vol. 65, no. 9, pp. 3818-3831, Sep. 2017.

[257] M. Ding, D. López-Pérez, G. Mao, and Z. Lin, "Performance impact of idle mode capability on dense small cell networks," IEEE Trans. Veh. Technol., vol. 66, no. 11, pp. 10446-10460, Nov. 2017.

[258] I. Bor-Yaliniz and H. Yanikomeroglu, "The new frontier in RAN heterogeneity: Multi-tier drone-cells," IEEE Commun. Mag., vol. 54, no. 11, pp. 48-55, Nov. 2016.

[259] O. M. Bushnaq, A. Çelik, H. Elsawy, M.-S. Alouini, and T. Y. AlNaffouri, "Aeronautical data aggregation and field estimation in IoT networks: hovering and traveling time dilemma of UAVs," IEEE Trans. Wireless Commun., vol. 18, no. 10, pp. 4620-4635, Oct. 2019.

[260] H. El Hammouti, M. Benjillali, B. Shihada, and M.-S. Alouini, "Learnas-you-fly: a distributed algorithm for joint 3D placement and user association in multi-UAVs networks," IEEE Trans. Wireless Commun., vol. 18, no. 12, pp. 5831-5844, Dec. 2019.

[261] Y. Qin, M. A. Kishk, and M.-S. Alouini, "Performance evaluation of UAV-enabled cellular networks with battery-limited drones," IEEE Commun. Lett., vol. 24, no. 12, pp. 2664-2668, Dec. 2020.

[262] M. Alzenad and H. Yanikomeroglu, "Coverage and rate analysis for vertical heterogeneous networks (VHetNets)," IEEE Trans. Wireless Commun., vol. 18, no. 12, pp. 5643-5657, Dec. 2019.

[263] M. Mozaffari, W. Saad, M. Bennis, and M. Debbah, "Unmanned aerial vehicle with underlaid device-to-device communications: performance and tradeoffs," IEEE Trans. Wireless Commun., vol. 15, no. 6, pp. 3949-3963, Jun. 2016.

[264] A. M. Hayajneh, S. A. R. Zaidi, D. C. McLernon, M. Di Renzo, and M. Ghogho, "Performance analysis of UAV enabled disaster recovery networks: A stochastic geometric framework based on cluster processes," IEEE Access, vol. 6, pp. 26215-26230, 2018.

[265] R. Ma, W. Yang, Y. Zhang, J. Liu, and H. Shi, "Secure mmWave communication using UAV-enabled relay and cooperative jammer," IEEE Access, vol. 7, pp. 119729-119741, Sep. 2019.

[266] O. M. Bushnaq, M. A. Kishk, A. Çelik, M.-S. Alouini, and T. Y. Al-Naffouri, "Optimal deployment of tethered drones for maximum cellular coverage in user clusters," IEEE Trans. Wireless Commun., doi: 10.1109/TWC.2020.3039013.

[267] Y. Hmamouche, M. Benjillali, S. Saoudi, and D. B. da Costa, "Uplink energy efficiency distribution with aerial users in cellular networks," IEEE Wireless Commun. Lett., vol. 10, no. 2, pp. 301-305, Feb. 2021.

[268] N. Bhushan et al., "Network densification: the dominant theme for wireless evolution into 5G," IEEE Commun. Mag., vol. 52, no. 2, pp. 82-89, Feb. 2014

[269] D. López-Pérez, M. Ding, H. Claussen, and A. H. Jafari, "Towards $1 \mathrm{Gbps} / \mathrm{UE}$ in cellular systems: understanding ultra-dense small cell deployments," IEEE Commun. Surveys Tuts., vol. 17, no. 4, pp. 20782101, Fourthquarter 2015.

[270] M. Kamel, W. Hamouda, and A. Youssef, "Ultra-dense networks: A survey," IEEE Commun. Surveys Tuts., vol. 18, no. 4, pp. 2522-2545, Fourthquarter 2016.

[271] D. Lopez-Perez, I. Guvenc, G. de la Roche, M. Kountouris, T. Q. S. Quek, and J. Zhang, "Enhanced intercell interference coordination challenges in heterogeneous networks," IEEE Wireless Commun., vol. 18, no. 3, pp. 22-30, Jun. 2011.

[272] I. Hwang, B. Song, and S. S. Soliman, "A holistic view on hyper-dense heterogeneous and small cell networks," IEEE Commun. Mag., vol. 51, no. 6, pp. 20-27, Jun. 2013.

[273] E. Bastug, M. Bennis, M. Kountouris, and M. Debbah, "Cache-enabled small cell networks: Modeling and tradeoffs," EURASIP J. Wireless Commun. Netw., vol. 2015, no. 1, p. 41, Feb. 2015.

[274] J. G. Andrews, H. Claussen, M. Dohler, S. Rangan, and M. C. Reed, "Femtocells: past, present, and future," IEEE J. Sel. Areas Commun., vol. 30, no. 3, pp. 497-508, Apr. 2012.

[275] T. Frisanco, P. Tafertshofer, P. Lurin, and R. Ang, "Infrastructure sharing and shared operations for mobile network operators: From a deployment and operations view," in Proc. IEEE Int. Conf. Commun. (ICC), pp. 2193-2200, Beijing, China, 2008.

[276] A. Khan, W. Kellerer, K. Kozu, and M. Yabusaki, "Network sharing in the next mobile network: TCO reduction, management flexibility, and operational independence," IEEE Commun. Mag., vol. 49, no. 10, pp. 134-142, Oct. 2011. 
[277] F. Boccardi et al., "Spectrum pooling in MmWave networks: opportunities, challenges, and enablers," IEEE Commun. Mag., vol. 54, no. 11, pp. 33-39, Nov. 2016.

[278] A. A. M. Saleh, A. J. Rustako, and R. S. Roman, 'Distributed antennas for indoor radio communications," IEEE Trans. Commun., vol. 35, pp. 1245-1251, Dec. 1987.

[279] J. Zhang and J. G. Andrews, "Distributed antenna systems with randomness," IEEE Trans. Wireless Commun., vol. 7, no. 9, pp. 36363646, Sep. 2008.

[280] Y. Lin and W. Yu, "Downlink spectral efficiency of distributed antenna systems under a stochastic model," IEEE Trans. on Wireless Commun., vol. 13, no. 12, pp. 6891-6902, Dec. 2014.

[281] M. Cheng and J. Wang, "Downlink transmission capacity analysis for virtual cell based distributed antenna systems," in Proc. IEEE Int. Conf. Commun. (ICC), pp. 1-6, France, Paris, 2017.

[282] X. Lin, R. W. Heath, and J. G. Andrews, "The interplay between massive MIMO and underlaid D2D networking," IEEE Trans. Wireless Commun., vol. 14, no. 6, pp. 3337-3351, Jun. 2015.

[283] A. Lo and I. Niemegeers, "Multi-hop relay architectures for 3GPP LTEadvanced," in Proc. IEEE 9th Int. Conf. Commun. (ICC), pp. 123-127, 2009, Kuala Lumpur, Malaysia.

[284] G. Liu, F. R. Yu, H. Ji, V. C. M. Leung, and X. Li, "In-band full-duplex relaying: A survey, research issues and challenges," IEEE Commun. Surveys Tuts, vol. 17, no. 2, pp. 500-524, Secondquarter 2015.

[285] J. He et al., "A Tutorial on lossy forwarding cooperative relaying," IEEE Commun. Surveys Tuts, vol. 21, no. 1, pp. 66-87, Firstquarter 2019.

[286] N. Nomikos et al., "A survey on buffer-aided relay selection," IEEE Commun. Surveys Tuts, vol. 18, no. 2, pp. 1073-1097, Secondquarter 2016.

[287] R. K. Ganti and M. Haenggi, "Spatial analysis of opportunistic downlink relaying in a two-hop cellular system," IEEE Trans. Commun., vol. 60, no. 5, pp. 1443-1450, May 2012.

[288] I. Krikidis, "Simultaneous information and energy transfer in largescale networks with/without relaying," IEEE Trans. Commun., vol. 62, no. 3, pp. 900-912, Mar. 2014.

[289] Z. Ding, I. Krikidis, B. Sharif, and H. V. Poor, "Wireless information and power transfer in cooperative networks with spatially random relays," IEEE Trans. on Wireless Commun., vol. 13, no. 8, pp. 44404453, Aug. 2014.

[290] J. Wen, M. Sheng, X. Wang, J. Li, and H. Sun, "On the capacity of downlink multi-hop heterogeneous cellular networks," IEEE Trans. Wireless Commun., vol. 13, no. 8, pp. 4092-4103, Aug. 2014.

[291] W. Lu and M. Di Renzo, "Stochastic geometry modeling and systemlevel analysis \& optimization of relay-aided downlink cellular networks," IEEE Trans. Commun., vol. 63, no. 11, pp. 4063-4085, Nov. 2015.

[292] K. Ntontin, M. Di Renzo, and C. Verikoukis, "On the feasibility of fullduplex relaying in multiple-antenna cellular networks," IEEE Trans. Commun., vol. 65, no. 5, pp. 2234-2249, May 2017.

[293] A. Asadi, Q. Wang, and V. Mancuso, "A survey on device-to-device communication in cellular networks," IEEE Commun. Surveys Tuts., vol. 16, no. 4, pp. 1801-1819, Fourthquarter 2014.

[294] F. Jameel, Z. Hamid, F. Jabeen, S. Zeadally, and M. A. Javed, "A survey of device-to-device communications: research issues and challenges," IEEE Commun. Surveys Tuts., vol. 20, no. 3, pp. 21332168, Thirdquarter 2018.

[295] X. Lin, J. G. Andrews, and A. Ghosh, "Spectrum sharing for deviceto-device communication in cellular networks," IEEE Trans. Wireless Commun., vol. 13, no. 12, pp. 6727-6740, Dec. 2014.

[296] F. Wu, H. Zhang, B. Di, J. Wu and L. Song, "Device-to-device communications underlaying cellular networks: To use unlicensed spectrum or not?," IEEE Trans. Commun., vol. 67, no. 9, pp. 6598-6611, Sept. 2019

[297] M. Peng, Y. Li, T. Q. S. Quek, and C. Wang, "Device-to-device underlaid cellular networks under Rician fading channels," IEEE Trans. Wireless Commun., vol. 13, no. 8, pp. 4247-4259, Aug. 2014.

[298] S. Andreev, O. Galinina, A. Pyattaev, K. Johnsson, and Y. Koucheryavy, "Analyzing assisted offloading of cellular user sessions onto D2D links in unlicensed bands," IEEE J. Sel. Areas Commun., vol. 33, no. 1, pp. 67-80, Jan. 2015.

[299] H. ElSawy, E. Hossain, and M.-S. Alouini, "Analytical modeling of mode selection and power control for underlay D2D communication in cellular networks," IEEE Trans. Commun., vol. 62, no. 11, pp. 41474161, Nov. 2014.
[300] H. V. Vu, N. H. Tran, and T. Le-Ngoc, "Full-duplex device-to-device cellular networks: power control and performance analysis," IEEE Trans. Veh. Technol., vol. 68, no. 4, pp. 3952-3966, Apr. 2019.

[301] V. Suryaprakash, P. Rost, and G. Fettweis, "Are heterogeneous cloudbased radio access networks cost effective?" IEEE J. Sel. Areas Commun., vol. 33, no. 10, pp. 2239-2251, Oct. 2015.

[302] F. A. Khan, H. He, J. Xue, and T. Ratnarajah, "Performance analysis of cloud radio access networks with distributed multiple antenna remote radio heads," IEEE Trans. Sig. Process., vol. 63, no. 18, pp. 4784-4799, Sep. 2015 .

[303] H. He, J. Xue, T. Ratnarajah, F. A. Khan, and C. B. Papadias, "Modeling and analysis of cloud radio access networks using Matérn hard-core point processes," IEEE Trans. Wireless Commun., vol. 15, no. 6, pp. 4074-4087, Jun. 2016.

[304] S. T. Veetil, K. Kuchi, and R. K. Ganti, "Coverage analysis of cloud radio networks with finite clustering," IEEE Trans. Wireless Commun., vol. 16, no. 1, pp. 594-606, Jan. 2017.

[305] J. Liu, M. Sheng, T. Q. S. Quek, and J. Li, "D2D enhanced coordinated multipoint in cloud radio access networks," IEEE Trans. Wireless Commun., vol. 15, no. 6, pp. 4248-4262, Jun. 2016.

[306] M. Mohammadi, H. A. Suraweera, and C. Tellambura, "Uplink/downlink rate analysis and impact of power allocation for fullduplex cloud-RANs," IEEE Trans. Wireless Commun., vol. 17, no. 9, pp. 5774-5788, Sep. 2018.

[307] X. Gu, X. Ji, Z. Ding, W. Wu, and M. Peng, "Outage probability analysis of non-orthogonal multiple access in cloud radio access networks," IEEE Commun. Lett., vol. 22, no. 1, pp. 149-152, Jan. 2018.

[308] M. Y. Arslan, K. Sundaresan, and S. Rangarajan, "Software-defined networking in cellular radio access networks: Potential and challenges," IEEE Commun. Mag., vol. 53, no. 1, pp. 150-156, Jan. 2015.

[309] H. Ishii, Y. Kishiyama, and H. Takahashi, "A novel architecture for LTE-B: C-plane/U-plane split and phantom cell concept," in Proc. IEEE Globecom Workshops, pp. 624-630, Anaheim, CA, USA, Dec. 2012.

[310] A. Zakrzewska, D. López-Pérez, S. Kucera, and H. Claussen, "Dual connectivity in LTE HetNets with split control- and user-plane," in Proc. IEEE Globecom Workshops (GC Wkshps), pp. 391-396, Atlanta, GA, USA, Dec. 2013.

[311] O. Semiari, W. Saad, M. Bennis, and M. Debbah, "Integrated millimeter wave and sub- $6 \mathrm{GHz}$ wireless networks: A roadmap for joint mobile broadband and ultra-reliable low-latency communications," IEEE Wireless Commun., vol. 26, no. 2, pp. 109-115, Apr. 2019.

[312] M. Peng, Y. Li, J. Jiang, J. Li, and C. Wang, "Heterogeneous cloud radio access networks: a new perspective for enhancing spectral and energy efficiencies," IEEE Wireless Commun., vol. 21, no. 6, pp. 126135, Dec. 2014.

[313] S. Mukherjee and H. Ishii, "Energy efficiency in the phantom cell enhanced local area architecture," in Proc. IEEE Wireless Commun. Netw. Conf. (WCNC), pp. 1267-1272, Shanghai, China, 2013.

[314] T. Han et al., "Small cell offloading through cooperative communication in software-defined heterogeneous networks," IEEE Sensors J., vol. 16, no. 20, pp. 7381-7392, Oct. 2016.

[315] H. Ibrahim, H. ElSawy, U. T. Nguyen, and M.-S. Alouini, "Mobilityaware modeling and analysis of dense cellular networks with $C$ plane/U-plane split architecture," IEEE Trans. Commun., vol. 64, no. 11, pp. 4879-4894, Nov. 2016.

[316] B. Yang, X. Yang, X. Ge, and Q. Li, "Coverage and handover analysis of ultra-dense millimeter-wave networks with control and user plane separation architecture," IEEE Access, vol. 6, pp. 54739-54750, 2018.

[317] R. Arshad, L. Lampe, H. ElSawy, and M. J. Hossain, "Integrating UAV into existing wireless networks: A stochastic geometry approach," in Proc. IEEE Globecom Workshops (GC Wkshps), pp. 1-6, Abu Dhabi, UAE, Dec. 2018.

[318] Y. Mao, C. You, J. Zhang, K. Huang, and K. B. Letaief, "A survey on mobile edge computing: The communication perspective," IEEE Commun. Surveys Tuts., vol. 19, no. 4, pp. 2322-2358, Fourthquarter 2017.

[319] H. Kong, I. Flint, P. Wang, D. Niyato, and N. Privault, "Fog radio access networks: Ginibre point process modeling and analysis," IEEE Trans. on Wireless Commun., vol. 17, no. 8, pp. 5564-5580, Aug. 2018.

[320] D. Malak, M. Al-Shalash, and J. G. Andrews, "Spatially correlated content caching for device-to-device communications," IEEE Trans. Wireless Commun., vol. 17, no. 1, pp. 56-70, Jan. 2018.

[321] B. Blaszczyszyn and A. Giovanidis, "Optimal geographic caching in cellular networks," in Proc. IEEE Int. Conf. Commun. (ICC), pp. 3358 3363, London, UK, 2015. 
[322] J. Wen, K. Huang, S. Yang, and V. O. K. Li, "Cache-enabled heterogeneous cellular networks: optimal tier-level content placement," IEEE Trans. Wireless Commun., vol. 16, no. 9, pp. 5939-5952, Sep. 2017.

[323] C. Yang, Y. Yao, Z. Chen, and B. Xia, "Analysis on cache-enabled wireless heterogeneous networks," IEEE Trans. Wireless Commun., vol. 15, no. 1, pp. 131-145, Jan. 2016

[324] Y. Cui, Y. Wu, and D. Jiang, "Analysis and optimization of caching and multicasting in large-scale cache-enabled information-centric networks," IEEE Glob. Commun. Conf. (GLOBECOM), pp. 1-7, San Diego, CA, USA, Dec. 2015.

[325] Z. Zhao, M. Peng, Z. Ding, W. Wang, and H. V. Poor, "Cluster content caching: an energy-efficient approach to improve quality of service in cloud radio access networks," IEEE J. Sel. Areas Commun., vol. 34 , no. 5, pp. 1207-1221, May 2016.

[326] M. R. Akdeniz et al., "Millimeter wave channel modeling and cellular capacity evaluation," IEEE J. Sel. Areas Commun., vol. 32, no. 6, pp. 1164-1179, Jun. 2014.

[327] G. Fontanesi, A. Zhu, and H. Ahmadi, "Outage analysis for millimeterwave fronthaul link of UAV-aided wireless networks," IEEE Access, vol. 8, pp. 111693-111706, Jun. 2020.

[328] J. Park, S. Kim, and J. Zander, "Tractable resource management with uplink decoupled millimeter-wave overlay in ultra-dense cellular networks," IEEE Trans. Wireless Commun., vol. 15, no. 6, pp. 43624379, Jun. 2016.

[329] H. Elshaer, M. N. Kulkarni, F. Boccardi, J. G. Andrews, and M. Dohler, "Downlink and uplink cell association with traditional macrocells and millimeter wave small cells," IEEE Trans. Wireless Commun., vol. 15 , no. 9, pp. 6244-6258, Sep. 2016.

[330] J. M. Jornet and I. F. Akyildiz, "Channel modeling and capacity analysis for electromagnetic wireless nanonetworks in the teraHertz band," IEEE Trans. Wireless Commun., vol. 10, no. 10, pp. 3211-3221, Oct. 2011.

[331] V. Petrov, M. Komarov, D. Moltchanov, J. M. Jornet, and Y. Koucheryavy, "Interference and SINR in millimeter wave and teraHertz communication systems with blocking and directional antennas," IEEE Trans. Wireless Commun., vol. 16, no. 3, pp. 1791-1808, Mar. 2017.

[332] J. Kokkoniemi, J. Lehtomäki, and M. Juntti, "Stochastic geometry analysis for mean interference power and outage probability in $\mathrm{THz}$ networks," IEEE Trans. Wireless Commun., vol. 16, no. 5, pp. 3017 3028, May 2017

[333] K. Ntontin and C. Verikoukis, "Toward the performance enhancement of microwave cellular networks through THz links," IEEE Trans. Veh. Technol., vol. 66, no. 7, pp. 5635-5646, Jul. 2017.

[334] J. Sayehvand and H. Tabassum, "Interference and coverage analysis in coexisting RF and dense teraHertz wireless networks," IEEE Wireless Commun. Lett., vol. 9, no. 10, pp. 1738-1742, Oct. 2020.

[335] C. Chaccour, M. N. Soorki, W. Saad, M. Bennis, and P. Popovski, "Can teraHertz provide high-rate reliable low latency communications for wireless VR?," arXiv preprint, May. 2020, [Online]. Available: https://arxiv.org/abs/2005.00536.

[336] C. Chen, D. A. Basnayaka, and H. Haas, "Downlink performance of optical attocell networks," J. Lightwave Technol., vol. 34, no. 1, pp. 137-156, Jan. 2016.

[337] L. Yin and H. Haas, "Coverage analysis of multiuser visible light communication networks," IEEE Trans. Wireless Commun., vol. 17, no. 3, pp. 1630-1643, Mar. 2018.

[338] H. Tabassum and E. Hossain, "Coverage and rate analysis for coexisting RF/VLC downlink cellular networks," IEEE Trans. Wireless Commun., vol. 17, no. 4, pp. 2588-2601, Apr. 2018.

[339] J. Kong, M. Ismail, E. Serpedin, and K. A. Qaraqe, "Energy efficient optimization of base station intensities for hybrid RF/VLC networks," IEEE Trans. Wireless Commun., vol. 18, no. 8, pp. 4171-4183, Aug. 2019

[340] G. Pan, J. Ye, and Z. Ding, "Secure hybrid VLC-RF systems with light energy harvesting," IEEE Trans. Commun., vol. 65, no. 10, pp. 4348-4359, Oct. 2017.

[341] L. Yin and H. Haas, "Physical-layer security in multiuser visible light communication networks," IEEE J. Sel. Areas Commun., vol. 36, no. 1, pp. 162-174, Jan. 2018.

[342] S. Cho, G. Chen, and J. P. Coon, "Securing visible light communication systems by beamforming in the presence of randomly distributed eavesdroppers," IEEE Trans. on Wireless Commun., vol. 17, no. 5, pp. 2918-2931, May 2018.

[343] M. A. Khalighi and M. Uysal, "Survey on free space optical communication: A communication theory perspective," IEEE Commun. Surveys Tuts., vol. 16, no. 4, pp. 2231-2258, Fourthquarter 2014.
[344] M. Lahmeri, M. A. Kishk, and M.-S. Alouini, "Stochastic Geometrybased analysis of Airborne Base Stations with Laser-powered UAVs," IEEE Commun. Lett., vol. 24, no. 1, pp. 173-177, Jan. 2020.

[345] F. Nadeem, V. Kvicera, M. S. Awan, E. Leitgeb, S. S. Muhammad, and G. Kandus, "Weather effects on hybrid FSO/RF communication link," IEEE J. Sel. Areas Commun., vol. 27, no. 9, pp. 1687-1697, Dec. 2009.

[346] A. Vavoulas, H. G. Sandalidis, and D. Varoutas, "Weather effects on FSO network connectivity," IEEE/OSA J. of Optical Commun. and Netw., vol. 4, no. 10, pp. 734-740, Oct. 2012.

[347] N. Letzepis and A. Guillen i Fabregas, "Outage probability of the freespace optical channel with doubly stochastic scintillation," IEEE Trans. Commun., vol. 57, no. 10, pp. 2899-2902, Oct. 2009.

[348] V. G. Sidorovich, "Solar background effects in wireless optical communications," in Proc. of SPIE, Optical Wireless Commun., vol. 4873, pp. 133-142, 2002

[349] S. Haykin, "Cognitive radio: brain-empowered wireless communications," IEEE J. Sel. Areas Commun., vol. 23, no. 2, pp. 201-220, Feb. 2005.

[350] U. Tefek and T. J. Lim, "Interference management through exclusion zones in two-tier cognitive networks," IEEE Trans. Wireless Commun. vol. 15, no. 3, pp. 2292-2302, Mar. 2016.

[351] X. Song, C. Yin, D. Liu, and R. Zhang, "Spatial throughput characterization in cognitive radio networks with threshold-based opportunistic spectrum access," IEEE J. Sel. Areas Commun., vol. 32, no. 11, pp. 2190-2204, Nov. 2014.

[352] J. Bang, J. Lee, S. Kim, and D. Hong, "An efficient relay selection strategy for random cognitive relay networks," IEEE Trans. Wireless Commun., vol. 14, no. 3, pp. 1555-1566, Mar. 2015.

[353] S. Cheng, W. C. Ao, F. Tseng, and K. Chen, "Design and analysis of downlink spectrum sharing in two-tier cognitive femto networks," IEEE Trans. Veh. Technol., vol. 61, no. 5, pp. 2194-2207, Jun. 2012.

[354] L. H. Afify, H. ElSawy, T. Y. Al-Naffouri, and M.-S. Alouini, "A unified stochastic geometry model for MIMO cellular networks with retransmissions," IEEE Trans. Wireless Commun., vol. 15, no. 12, pp. 8595-8609, Dec. 2016.

[355] X. Yu, C. Li, J. Zhang, M. Haenggi, and K. B. Letaief, "A unified framework for the tractable analysis of multi-antenna wireless networks," IEEE Trans. Wireless Commun., vol. 17, no. 12, pp. 7965-7980, Dec. 2018.

[356] E. Björnson, L. Sanguinetti, and M. Kountouris, "Deploying dense networks for maximal energy efficiency: small cells meet massive MIMO," IEEE J. Sel. Areas Commun., vol. 34, no. 4, pp. 832-847, Apr. 2016.

[357] D. Verenzuela, E. Björnson, and L. Sanguinetti, "Spectral and energy efficiency of superimposed pilots in uplink massive MIMO," EEE Trans. Wireless Commun., vol. 17, no. 11, pp. 7099-7115, Nov. 2018.

[358] P. Parida and H. S. Dhillon, "Stochastic geometry-based uplink analysis of massive MIMO systems with fractional pilot reuse," IEEE Trans. Wireless Commun., vol. 18, no. 3, pp. 1651-1668, Mar. 2019.

[359] M. Di Renzo et al., "Smart radio environments empowered by reconfigurable intelligent surfaces: how It works, state of research, and the road ahead," IEEE J. Sel. Areas Commun., vol. 38, no. 11, pp. 2450-2525, Nov. 2020

[360] S. Alfattani, W. Jaafar, Y. Hmamouche, H. Yanikomeroglu, A. Yongaçoglu, N. D. Dào, and P. Zhu, "Aerial platforms with reconfigurable smart surfaces for $5 \mathrm{G}$ and beyond," IEEE Commun. Mag., vol. 59, no. 1, pp. 96-102, Jan. 2021.

[361] M. Di Renzo et al., "Reconfigurable intelligent surfaces vs. relaying: differences, similarities, and performance comparison," IEEE Open $J$. Commun. Soc., vol. 1, pp. 798-807, 2020.

[362] M. Di Renzo and J. Song, "Reflection probability in wireless networks with metasurface-coated environmental objects: an approach based on random spatial processes," EURASIP J. Wireless Commun. Netw., Vol. 99, pp. 1-15, Apr. 2019

[363] M. A. Kishk and M.-S. Alouini, "Exploiting randomly-located blockages for large-scale deployment of intelligent surfaces," IEEE J. Sel. Areas Commun., doi: 10.1109/JSAC.2020.3018808.

[364] E. Björnson and L. Sanguinetti, "Power scaling laws and near-field behaviors of massive MIMO and intelligent reflecting surfaces," IEEE Open J. Commun. Soc., vol. 1, pp. 1306-1324, Sep. 2020.

[365] F. H. Danufane, M. Di Renzo, J. D. Rosny, and S. Tretyakov, "On the path-loss of reconfigurable intelligent surfaces: an approach based on Green's theorem applied to vector fields," arXiv preprint, Jul. 2020, [Online]. Available: https://arxiv.org/abs/2007.13158

[366] M. Di Renzo, F. Habibi Danufane, X. Xi, J. D. Rosny, and S. Tretyakov, "Analytical modeling of the path-loss for reconfigurable intelligent surfaces - anomalous mirror or scatterer ?," in Proc. IEEE 21st Int. 
Workshop Signal Process. Adv. Wireless Commun. (SPAWC), pp. 1-5, Atlanta, GA, USA, May 2020.

[367] W. Tang et al., "Path loss modeling and measurements for reconfigurable intelligent surfaces in the millimeter-wave frequency band," arXiv preprint, Jan. 2021, [Online]. Available: https://arxiv.org/abs/2101.08607.

[368] W. Tang et al., "Wireless communications with reconfigurable intelligent surface: path loss modeling and experimental measurement," IEEE Trans. Wireless Commun., vol. 20, no. 1, pp. 421-439, Jan. 2021.

[369] G. Gradoni and M. Di Renzo, "End-to-end mutual coupling aware communication model for reconfigurable intelligent surfaces: an electromagnetic-compliant approach based on mutual impedances," arXiv preprint, Dec. 2020, [Online]. Available: https://arxiv.org/abs/2009.02694v2.

[370] J. C. B. Garcia, A. Sibille, and M. Kamoun, "Reconfigurable intelligent surfaces: bridging the gap between scattering and reflection," IEEE $J$. Sel. Areas Commun., vol. 38, no. 11, pp. 2538-2547, Nov. 2020.

[371] M. Najafi, V. Jamali, R. Schober, and H. V. Poor, "Physics-based modeling and scalable optimization of large intelligent reflecting surfaces," IEEE Trans. Commun., doi: 10.1109/TCOMM.2020.3047098.

[372] D. Dardari, "Communicating with large intelligent surfaces: fundamental limits and models," IEEE J. Sel. Areas Commun., vol. 38, no. 11, pp. 2526-2537, Nov. 2020.

[373] X. Qian and M. Di Renzo, "Mutual coupling and unit cell aware optimization for reconfigurable intelligent surfaces," arXiv preprint, Nov. 2020, [Online]. Available: https://arxiv.org/abs/2011.14373.

[374] N. S. Perović, L. -N. Tran, M. Di Renzo, and M. F. Flanagan, "Achievable rate optimization for MIMO systems with reconfigurable intelligent surfaces," IEEE Trans. Wireless Commun., doi: 10.1109/TWC.2021.3054121.

[375] A. Abrardo, D. Dardari, and M. Di Renzo, "Intelligent reflecting surfaces: sum-rate optimization based on statistical CSI," arXiv preprint, Dec. 2020, [Online]. Available: https://arxiv.org/abs/2012.10679.

[376] Y. Saito, Y. Kishiyama, A. Benjebbour, T. Nakamura, A. Li, and K. Higuchi, "Non-orthogonal multiple access (NOMA) for cellular future radio access," in Proc. IEEE 77th Veh. Technol. Conf. (VTC Spring), pp. 1-5, Dresden, Germany, 2013.

[377] H. Tabassum, E. Hossain, and J. Hossain, "Modeling and analysis of uplink non-orthogonal multiple access in large-scale cellular networks using poisson cluster processes," IEEE Trans. Commun., vol. 65, no. 8, pp. 3555-3570, Aug. 2017.

[378] Y. Liu, Z. Qin, M. Elkashlan, Y. Gao, and A. Nallanathan, "Nonorthogonal multiple access in massive MIMO aided heterogeneous networks," in Proc. IEEE Glob. Commun. Conf. (GLOBECOM), pp. 1-6, Washington, DC, USA, Dec. 2016

[379] Z. Zhang, H. Sun, and R. Q. Hu, "Downlink and uplink non-orthogonal multiple access in a dense wireless network," IEEE J. Sel. Areas Commun., vol. 35, no. 12, pp. 2771-2784, Dec. 2017.

[380] F. J. Martin-Vega, Y. Liu, G. Gomez, M. C. Aguayo-Torres, and M. Elkashlan, "Modeling and analysis of NOMA enabled CRAN with cluster point process," in Proc. Glob. Commun. Conf. (GLOBECOM), pp. 1-6, Singapore, Dec. 2017.

[381] X. Gu, X. Ji, Z. Ding, W. Wu, and M. Peng, "Outage probability analysis of non-orthogonal multiple access in cloud radio access networks," IEEE Commun. Lett., vol. 22, no. 1, pp. 149-152, Jan. 2018.

[382] C. Liu and D. Liang, "Heterogeneous networks with power-domain NOMA: coverage, throughput, and power allocation analysis," IEEE Trans. Wireless Commun., vol. 17, no. 5, pp. 3524-3539, May 2018.

[383] Z. Zhang, Z. Ma, M. Xiao, X. Lei, Z. Ding, and P. Fan, "Fundamental tradeoffs of non-orthogonal multicast, multicast, and unicast in ultradense networks," IEEE Trans. Commun., vol. 66, no. 8, pp. 3555-3570, Aug. 2018

[384] T. Hou, Y. Liu, Z. Song, X. Sun, and Y. Chen, "Multiple antenna aided NOMA in UAV networks: A stochastic geometry approach," IEEE Trans. Commun., vol. 67, no. 2, pp. 1031-1044, Feb. 2019.

[385] P. Swami, V. Bhatia, S. Vuppala, and T. Ratnarajah, "On user offloading in NOMA-hetnet using repulsive point process," IEEE Systems J., vol. 13, no. 2, pp. 1409-1420, Jun. 2019.

[386] K. Ali, H. Elsawy, and M.-S. Alouini, "Meta distribution of downlink non-orthogonal multiple access (NOMA) in Poisson networks," IEEE Wireless Communications Letters, vol. 8, no. 2, pp. 572-575, Apr. 2019.

[387] K. Ali, H. Elsawy, and M.-S. Alouini, "Meta distribution of downlink non-orthogonal multiple access (NOMA) in Poisson networks," IEEE Wireless Commun. Lett., vol. 8, no. 2, pp. 572-575, Apr. 2019.

[388] M. Salehi, H. Tabassum, and E. Hossain, "Meta distribution of SIR in large-scale uplink and downlink NOMA networks," IEEE Trans. on Commun., vol. 67, no. 4, pp. 3009-3025, Apr. 2019.
[389] J. Lee and T. Q. S. Quek, "Hybrid full-/half-duplex system analysis in heterogeneous wireless networks," IEEE Trans. Wireless Commun. vol. 14, no. 5, pp. 2883-2895, May 2015.

[390] Z. Tong and M. Haenggi, "Throughput analysis for full-duplex wireless networks with imperfect self-interference cancellation," IEEE Trans. Commun., vol. 63, no. 11, pp. 4490-4500, Nov. 2015.

[391] H. Tabassum, A. H. Sakr, and E. Hossain, "Analysis of massive MIMOenabled downlink wireless backhauling for full-duplex small cells," IEEE Trans. Commun., vol. 64, no. 6, pp. 2354-2369, Jun. 2016.

[392] K. Ntontin, M. Di Renzo, and C. Verikoukis, "On the feasibility of fullduplex relaying in multiple-antenna cellular networks," IEEE Trans. Commun., vol. 65, no. 5, pp. 2234-2249, May 2017.

[393] A. H. Sakr and E. Hossain, "On user association in multi-tier fullduplex cellular networks," IEEE Trans. Commun., vol. 65, no. 9, pp. 4080-4095, Sep. 2017.

[394] A. Shojaeifard, K. Wong, M. Di Renzo, G. Zheng, K. A. Hamdi, and J. Tang, "Massive MIMO-enabled full-duplex cellular networks," IEEE Trans. on Commun., vol. 65, no. 11, pp. 4734-4750, Nov. 2017.

[395] A. Sharma, R. K. Ganti, and J. K. Milleth, "Joint backhaul-access analysis of full duplex self-backhauling heterogeneous networks," IEEE Trans. Wireless Commun., vol. 16, no. 3, pp. 1727-1740, Mar. 2017.

[396] I. Atzeni and M. Kountouris, "Full-duplex MIMO small-cell networks with interference cancellation," IEEE Trans. Wireless Commun., vol. 16, no. 12, pp. 8362-8376, Dec. 2017.

[397] X. Zhou, R. K. Ganti, J. G. Andrews, and A. Hjorungnes, "On the throughput cost of physical layer security in decentralized wireless networks," IEEE Trans. Wireless Commun., vol. 10, no. 8, pp. 2764 2775, Aug. 2011.

[398] H. Wang, X. Zhou, and M. C. Reed, "Physical layer security in cellular networks: A stochastic geometry approach," IEEE Trans. Wireless Commun., vol. 12, no. 6, pp. 2776-2787, Jun. 2013.

[399] H. Wu, X. Tao, N. Li, and J. Xu, "Secrecy outage probability in multiRAT heterogeneous networks," IEEE Commun. Lett., vol. 20, no. 1, pp. 53-56, Jan. 2016.

[400] H. Wang, T. Zheng, J. Yuan, D. Towsley, and M. H. Lee, "Physical layer security in heterogeneous cellular networks," IEEE Trans. on Commun., vol. 64, no. 3, pp. 1204-1219, Mar. 2016.

[401] Y. Liu, Z. Qin, M. Elkashlan, Y. Gao, and L. Hanzo, "Enhancing the physical layer security of non-orthogonal multiple access in large-scale networks," IEEE Trans. Wireless Commun., vol. 16, no. 3, pp. 16561672, Mar. 2017.

[402] W. Wang, K. C. Teh, and K. H. Li, "Artificial noise aided physical layer security in multi-antenna small-cell networks," IEEE Trans. Inf. Forensics and Security, vol. 12, no. 6, pp. 1470-1482, Jun. 2017.

[403] C. Ma, J. Liu, X. Tian, H. Yu, Y. Cui, and X. Wang, "Interference exploitation in D2D-enabled cellular networks: A secrecy perspective," IEEE Trans. Commun., vol. 63, no. 1, pp. 229-242, Jan. 2015.

[404] S. Vuppala, S. Biswas, and T. Ratnarajah, "An analysis on secure communication in millimeter/micro-wave hybrid networks," IEEE Trans. Commun., vol. 64, no. 8, pp. 3507-3519, Aug. 2016.

[405] H. Wang, C. Wang, T. Zheng, and T. Q. S. Quek, "Impact of artificial noise on cellular networks: A stochastic geometry approach," IEEE Trans. Wireless Commun., vol. 15, no. 11, pp. 7390-7404, Nov. 2016.

[406] W. Tang, S. Feng, Y. Ding, and Y. Liu, "Physical layer security in heterogeneous networks With jammer selection and full-duplex users," IEEE Trans. Wireless Commun., vol. 16, no. 12, pp. 7982-7995, Dec. 2017.

[407] H. Kong, P. Wang, D. Niyato, and Y. Cheng, "Physical layer security in wireless networks with Ginibre point processes," IEEE Trans. Wireless Commun., vol. 17, no. 8, pp. 5132-5147, Aug. 2018.

[408] G. Chen, J. P. Coon, and M. Di Renzo, "Secrecy outage analysis for downlink transmissions in the presence of randomly located eavesdroppers," IEEE Trans. Inf. Forensics Security, vol. 12, no. 5, pp. 11951206, May 2017.

[409] N. Rikhtegar and M. Keshtgary, "A brief survey on molecular and electromagnetic communications in nano-networks," Int. J. Comput. Appl., vol. 79, pp. 16-28, Oct. 2013.

[410] N. Farsad, H. B. Yilmaz, A. Eckford, C. Chae, and W. Guo, "A comprehensive survey of recent advancements in molecular communication," IEEE Commun. Surveys Tuts, vol. 18, no. 3, pp. 1887-1919, Thirdquarter 2016.

[411] N. Farsad, W. Guo, and A. W. Eckford, "Tabletop molecular communication: Text messages through chemical signals," PLoS One, vol. 8 , Dec. 2013.

[412] Y. Deng, A. Noel, W. Guo, A. Nallanathan, and M. Elkashlan, "Analyzing large-scale multiuser molecular communication via 3-D 
stochastic geometry," IEEE Trans. Molecular, Biological and MultiScale Commun., vol. 3, no. 2, pp. 118-133, Jun. 2017.

[413] W. Guo, C. Mias, N. Farsad, and J. Wu, "Molecular versus electromagnetic wave propagation loss in macro-scale environments," IEEE Trans. Molecular, Biological and Multi-Scale Commun., vol. 1, no. 1, pp. 18-25, Mar. 2015.

[414] O. Simeone, "A very brief introduction to machine learning with applications to communication systems," IEEE Trans. on Cogn. Commun. Netw., vol. 4, no. 4, pp. 648-664, Dec. 2018.

[415] Y. Sun, M. Peng, Y. Zhou, Y. Huang, and S. Mao, "Application of machine learning in wireless networks: key techniques and open issues," IEEE Commun. Surveys Tuts, vol. 21, no. 4, pp. 3072-3108, Fourthquarter 2019.

[416] H. E. Hammouti, M. Ghogho, and S. A. Raza Zaidi, "A machine learning approach to predicting coverage in random wireless networks," in Proc. IEEE Globecom Workshops (GC Wkshps), pp. 1-6, Abu Dhabi, UAE, Dec. 2018.

[417] M. Di Renzo, A. Zappone, T. T. Lam, and M. Debbah, "Spectral-energy efficiency Pareto front in cellular networks: a stochastic geometry framework," IEEE Wireless Commun. Lett., vol. 8, no. 2, pp. 424-427, April 2019

[418] J. Song, M. Di Renzo, A. Zappone, V. Sciancalepore, and X. C. Perez, "System-level optimization in Poisson cellular networks: an approach based on the generalized benders decomposition," IEEE Wireless Commun. Lett., vol. 9, no. 10, pp. 1773-1777, Oct. 2020.

[419] J. Schloemann, H. S. Dhillon, and R. M. Buehrer, "Toward a tractable analysis of localization fundamentals in cellular networks," IEEE Trans. Wireless Commun., vol. 15, no. 3, pp. 1768-1782, Mar. 2016.

[420] C. E. O'Lone, H. S. Dhillon, and R. M. Buehrer, "A statistical characterization of localization performance in wireless networks," IEEE Trans. Wireless Commun., vol. 17, no. 9, pp. 5841-5856, Sept. 2018

[421] B. Vo, B. Vo, N. Pham, and D. Suter, "Joint detection and estimation of multiple objects from image observations," IEEE Trans Signal Process., vol. 58, no. 10, pp. 5129-5141, Oct. 2010.

[422] J. Mullane, B. Vo, M. D. Adams, and B. Vo, "A random-finite-set approach to bayesian SLAM," IEEE Trans. Robot., vol. 27, no. 2, pp. 268-282, Apr. 2011.

[423] A. Grothendieck, "Récoltes et Semailles". [Online]. Available: https://www.quarante-deux.org/archives/klein/prefaces/Romans_19651969/Recoltes_et_semailles.pdf.

[424] O. Caramello, "The unification of mathematics via topos theory," arXiv preprint, Jun. 2010, [Online]. Available: https://arxiv.org/abs/1006.3930.

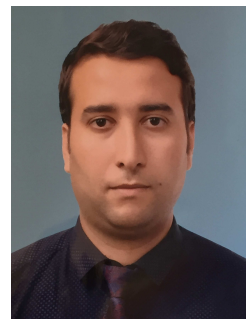

Yassine Hmamouche (M'19) received his Ph.D. degree in telecommunications from IMT Atlantique, Brest, France, in 2020, where he is currently a Postdoctoral Fellow. Prior to joining academia, he extensively worked for eight years in the telecom industry as a radio network planning engineer and then as a project manager for several projects spanning different technologies. In 2019, he was a Visiting Researcher with Carleton University, Ottawa, Canada. His current research interests include UAV communication systems, reconfigurable intelligent surfaces, and IoT networks.

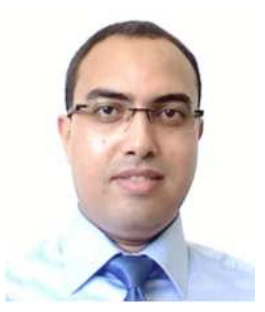

Mustapha Benjillali (SM'14) received the Ph.D. degree in telecommunications from INRS, Montreal, Canada, in 2009. He was a post-doctoral research fellow with the Electrical Engineering Program, King Abdullah University of Science and Technology (KAUST), Saudi Arabia. He is currently a full professor with the Communications Systems Department, INPT, Rabat, Morocco, where he is the research team leader on Trends in Research on 5G-and-Beyond Technologies and Eco-Systems (5TRonG). He serves on the editorial board of many leading international journals, and in different organizational and TPC chairing roles in several major international IEEE conferences. His current research interests are in the broad area of wireless communications for $6 \mathrm{G}$ applications, with a focus on mathematical and stochastic modeling, machine learning for communications, performance analysis, and optimal resource allocation strategies.

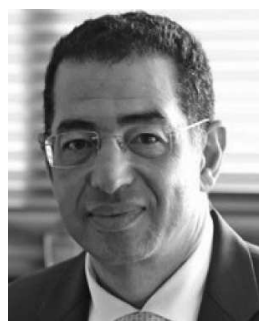

Samir Saoudi (SM'09) was born in Rabat, Morocco, in 1963. He received the Electrical Engineering degree from ENST-Bretagne in 1987, the $\mathrm{Ph} . \mathrm{D}$. degree in telecommunications from Rennes I University in 1990, and the HDR Qualification degree as a Supervisor for Ph.D. students in 1997. Since 1991, he has been with the Signal Communications Department, IMT Atlantique (Telecom Bretagne) where he is currently a Professor. His research interests cover the areas of non parametric probability density estimation, multi-user detection, MIMO systems, and iterative processing with applications to wireless communications. He has co-authored over 35 journal papers and 100 conference papers, and supervised over $35 \mathrm{Ph} . \mathrm{D}$. Students. Since 1995, he has been the Head of the Master Digital Communications Systems. He has been the General Chair of the second International symposium on Image/Video Communications over fixed and mobile networks, in 2004. He has been the Co-Chair of the MIMO Systems Symposium, the IEEE International Wireless Communications and Mobile Computing Conference, IWCMC 2010, France. Since 2008, he has been the Head of the Digital Communications Group, LabSticc, CNRS UMR 6385. From 2014 to 2020, he was the Head of the Signal \& Communications Department. Since 2021, he has been the Head of the Mathematical \& Electrical Engineering Department, IMT Atlantique.

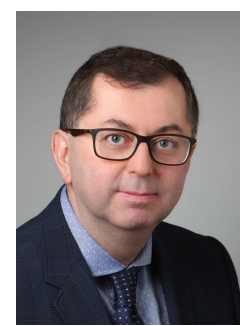

Halim Yanikomeroglu (F'17) is a full professor in the Department of Systems and Computer Engineering at Carleton University, Ottawa, Canada. His research interests cover many aspects of $5 \mathrm{G} / 5 \mathrm{G}+$ wireless networks. His collaborative research with industry has resulted in 37 granted patents. He is a Fellow of IEEE, Engineering Institute of Canada (EIC), and Canadian Academy of Engineering (CAE); he is a Distinguished Speaker for IEEE Communications Society and IEEE Vehicular Technology Society. He is currently serving as the Chair of the IEEE WCNC (Wireless Communications and Networking Conference) Steering Committee. He was the Technical Program Chair/Co-Chair of WCNC 2004 (Atlanta), WCNC 2008 (Las Vegas), and WCNC 2014 (Istanbul). He was the General Chair of IEEE VTC 2010-Fall (Ottawa) and VTC 2017-Fall (Toronto). He also served as the Chair of the IEEE's Technical Committee on Personal Communications. Dr. Yanikomeroglu received several awards for his research, teaching, and service, including the IEEE Communications Society Wireless Communications Technical Committee Recognition Award in 2018. 


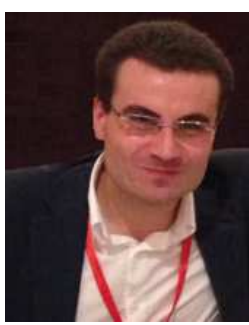

Marco Di Renzo (F'20) received the Laurea (cum laude) and Ph.D. degrees in electrical engineering from the University of L'Aquila, Italy, in 2003 and 2007, respectively, and the Habilitation à Diriger des Recherches (Doctor of Science) degree from University Paris-Sud, France, in 2013. Since 2010, he has been with the French National Center for Scientific Research (CNRS), where he is a CNRS Research Director (CNRS Professor) in the Laboratory of Signals and Systems (L2S) of Paris-Saclay University CNRS and CentraleSupelec, Paris, France. In ParisSaclay University, he serves as the Coordinator of the Communications and Networks Research Area of the Laboratory of Excellence DigiCosme, and as a member of the Admission and Evaluation Committee of the Ph.D. School on Information and Communication Technologies. Currently, he serves as the Editor-in-Chief of IEEE Communications Letters, and is a Distinguished Speaker of the IEEE Vehicular Technology Society. In 2017-2020, he was a Distinguished Lecturer of the IEEE Vehicular Technology Society and IEEE
Communications Society. He served as an Editor and the Associate Editor-inChief of IEEE Communications Letters, and as an Editor of IEEE Transactions on Communications and IEEE Transactions on Wireless Communications. Also, he serves as the Founding Chair of the Special Interest Group on Reconfigurable Intelligent Surfaces of the Wireless Technical Committee of the IEEE Communications Society, and is the Founding Lead Editor of the IEEE Communications Society Best Readings in Reconfigurable Intelligent Surfaces. He is a Highly Cited Researcher (Clarivate Analytics, Web of Science), a World's Top 2\% Scientist from Stanford University, a Fellow of the IEEE, and a Fellow of the IET. He has received several individual distinctions and research awards, which include the IEEE Communications Society Best Young Researcher Award for Europe, Middle East and Africa, the Royal Academy of Engineering Distinguished Visiting Fellowship, the IEEE Jack Neubauer Memorial Best System Paper Award, the IEEE Communications Society Young Professional in Academia Award, the SEE-IEEE Alain Glavieux Award, and a 2019 IEEE ICC Best Paper Award. In 2019, he was a recipient of a Nokia Foundation Visiting Professorship for conducting research on metamaterial-assisted wireless communications at Aalto University, Finland. 